\title{
Mechanistic studies of the ethylene trimerization reaction with chromium - diphosphine catalysts: experimental evidence for a mechanism involving metallacyclic intermediates
}

\author{
Theodor Agapie, Susan J. Schofer, Jay A. Labinger, ${ }^{*}$ and John E. Bercaw* \\ Arnold and Mabel Beckman Laboratories of Chemical Synthesis, California Institute of Technology, \\ Pasadena, California 91125 \\ RECEIVED DATE (automatically inserted by publisher); Email: jal@caltech.edu; bercaw@caltech.edu
}

\section{Supporting Information}

Table of Contents

S1. Experimental Section ........................................................ 4

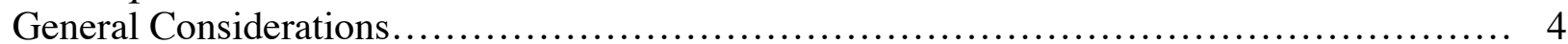

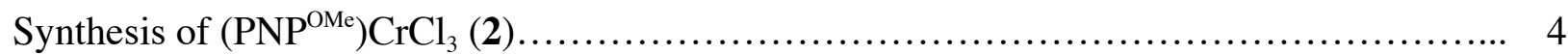

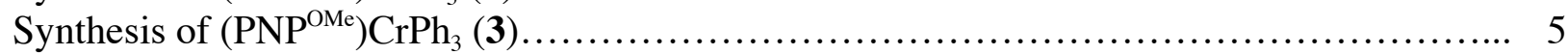

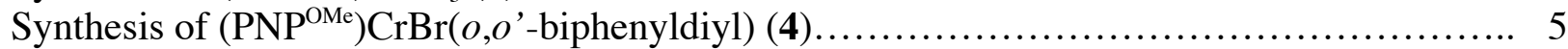

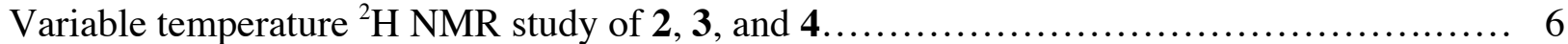

General procedure for trimerization of $\mathrm{C}_{2} \mathrm{H}_{4}$ with 3 activated with $\mathrm{H}\left(\mathrm{Et}_{2} \mathrm{O}\right)_{2} \mathrm{~B}_{[}\left[\mathrm{C}_{6} \mathrm{H}_{3}\left(\mathrm{CF}_{3}\right)_{2}\right]_{4} \ldots 6$

Trimerization of a $\mathrm{C}_{2} \mathrm{D}_{4} / \mathrm{C}_{2} \mathrm{H}_{4}$ mixture with 3 activated with $\mathrm{H}\left(\mathrm{Et}_{2} \mathrm{O}\right)_{2} \mathrm{~B}\left[\mathrm{C}_{6} \mathrm{H}_{3}\left(\mathrm{CF}_{3}\right)_{2}\right]_{4} \ldots \ldots \ldots . \quad 8$

Trimerization of a $\mathrm{C}_{2} \mathrm{D}_{4} / \mathrm{C}_{2} \mathrm{H}_{4}$ mixture with 4 activated with $\mathrm{NaB}\left[\mathrm{C}_{6} \mathrm{H}_{3}\left(\mathrm{CF}_{3}\right)_{2}\right]_{4} \ldots \ldots \ldots \ldots \ldots . \quad 8$

Trimerization of a $\mathrm{C}_{2} \mathrm{D}_{4} / \mathrm{C}_{2} \mathrm{H}_{4}$ mixture with 4 activated with MAO...................... 8

Trimerization of a $\mathrm{C}_{2} \mathrm{D}_{4} / \mathrm{C}_{2} \mathrm{H}_{4}$ mixture with $\mathrm{CrCl}_{3}(\mathrm{THF})_{3} / \mathrm{PNP}^{\mathrm{OMe}}$ (1) activated with MAO... 9

Reaction of 4 with ethylene........................................................ 9

Trimerization of cis-, trans-, and gem- $\mathrm{C}_{2} \mathrm{H}_{2} \mathrm{D}_{2}$ with 4 activated with $\mathrm{NaB}\left[\mathrm{C}_{6} \mathrm{H}_{3}\left(\mathrm{CF}_{3}\right)_{2}\right]_{4} \ldots \ldots \ldots 10$

Table 1. Isotope effects for the trimerization of different isomers of dideuteroethylene under various reaction conditions. ..................................................... 11

Figure 1. ${ }^{1} \mathrm{H} \mathrm{NMR}$ data $\left(\mathrm{CD}_{2} \mathrm{Cl}_{2}, 500 \mathrm{MHz}\right)$ for the olefinic protons of various isotopomers of 1-

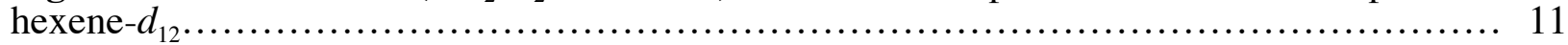

S2. Cossee-type and redox mechanisms for the formation of 1-hexene from ethylene. Expected outcomes and experimental data for the trimerization of a 1:1

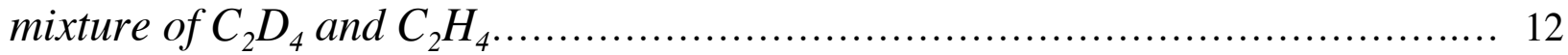

Scheme 1. Proposed Cossee-type mechanism for 1-hexene formation from ethylene........... 12 Figure 2. Some hexenes that could form via a Cossee-type mechanism (top). List of all expected isotopomers and their statistical distribution for a Cossee-type mechanism (bottom).......... 12 Figure 3. Proposed mechanism for 1-hexene formation from ethylene involving metallacyclic intermediates (top) and expected isotopomer distribution (bottom) ....................... 13 Figure 4. GC data of the 1-hexene fraction obtained from trimerization of a 1:1 mixture of $\mathrm{C}_{2} \mathrm{D}_{4}$

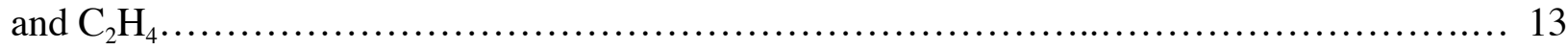


Figure 5. MS (red) data of the 1-hexene fraction obtained from trimerization of a 1:1 mixture of $\mathrm{C}_{2} \mathrm{D}_{4}$ and $\mathrm{C}_{2} \mathrm{H}_{4}$. Simulations of expected isotopomer distributions for a mechanism involving metallacyclic intermediates and for a Cossee-type mechanism are presented in green and blue, respectively.................................................................. 14

S3. Trimerization of cis-, trans- and gem-dideuteroethylene $-{ }^{1} \mathrm{H}$ NMR spectra of the volatile fraction .................................................................. 15

Figure 6. Olefinic region of the ${ }^{1} \mathrm{H}$ NMR spectrum of the volatile materials resulting from the trimerization of cis-dideuteroethylene............................................ 15

Figure 7. Olefinic region of the ${ }^{1} \mathrm{H}$ NMR spectrum of the volatile materials resulting from the trimerization of trans-dideuteroethylene ........................................... 16

Figure 8. Olefinic region of the ${ }^{1} \mathrm{H}$ NMR spectrum of the volatile materials resulting from the trimerization of gem-dideuteroethylene.

S4. Expected isotopomeric isomers and isotope effects for the trimerization of cisand gem-dideuteroethylene depending on the mechanism......................... 18 Figure 9. Possible isotopomeric isomers of 1-hexene from mechanism involving 2,1 re-insertion that is competitive with reductive elimination.

Figure 10. Proposed mechanisms involving reversible formation of the metallacycloheptane $v s$. subsequent steps (top). Expected isotope effect for the trimerization of gem-dideuteroethylene would correspond to the one observed for the trimerization of cis-or trans-dideuteroethylene, $i$. $e$. coming from the $\square$-hydride abstraction / reductive elimination steps (bottom). ............ 19 Figure 11. Expected isotopomer distribution from the trimerization of gem-dideuteroethylene for a mechanism involving irreversible formation of the metallacycloheptane and equivalent ends of

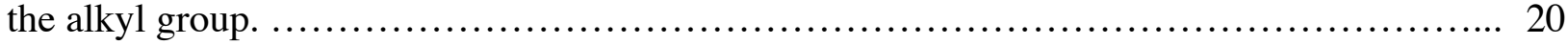
Figure 12. Expected isotopomer distribution from the trimerization of gem-dideuteroethylene for a mechanism involving irreversible formation of the metallacycloheptane and selective $\square$-hydride abstraction / reductive elimination from only one end of the dialkyl group. ................. 20

Figure 13. Drawing of the proposed metallacycloheptane intermediate displaying inequivalent

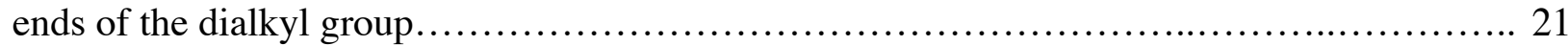

S5. Structural drawings, tables with structural parameters, and crystallographic information for 2,3 , and 4 .................................................. 21

Figure 14. Structural drawing of 2 with thermal ellipsoids at the 50\% probability level. ...... 21

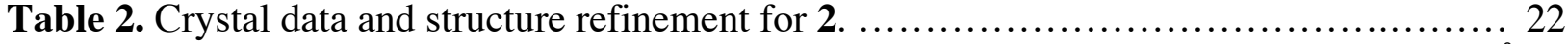

Table 3. Atomic coordinates $\left(\mathrm{x} 10^{4}\right)$ and equivalent isotropic displacement parameters $\left(\AA^{2} \mathrm{x}\right.$ $10^{3}$ ) for 2. 23

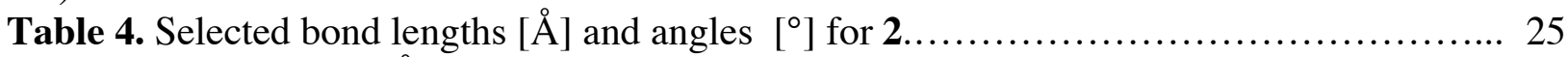

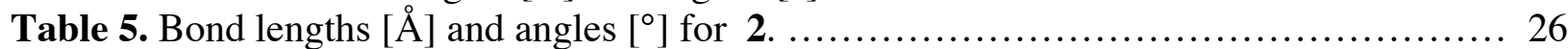

Table 6. Anisotropic displacement parameters $\left(\AA^{2}\right.$ x $\left.10^{4}\right)$ for 2 ............................. 29

Figure 15. Structural drawing of $\mathbf{3}$ with thermal ellipsoids at the $50 \%$ probability level. ...... 30

Table 7. Crystal data and structure refinement for 3. .............................. 31

Table 8. Atomic coordinates $\left(x 1^{4}\right)$ and equivalent isotropic displacement parameters $\left(\AA^{2} \mathrm{x}\right.$

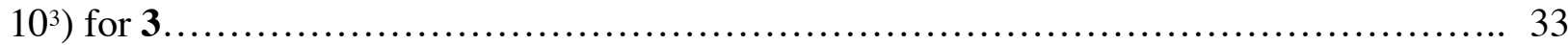

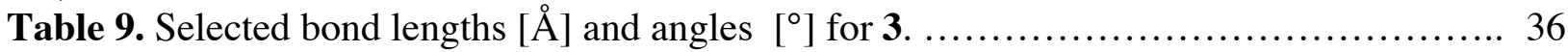




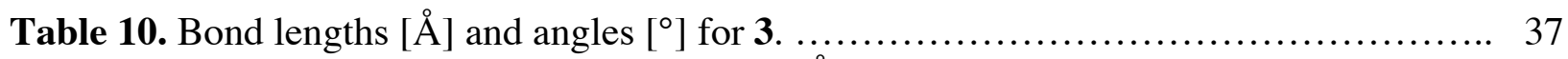

Table 11. Anisotropic displacement parameters $\left(\AA^{2} \times 10^{4}\right)$ for 3..................... 41

Figure 16. Structural drawing of $\mathbf{4}$ with thermal ellipsoids at the 50\% probability level........ 44

Table 12. Crystal data and structure refinement for 4 ................................ 45

Table 13. Atomic coordinates $\left(x 0^{4}\right)$ and equivalent isotropic displacement parameters $\left(\AA^{2} \mathrm{X}\right.$

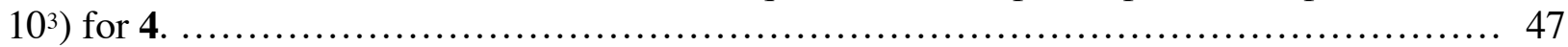

Table 14. Selected bond lengths $[\AA]$ and angles $\left[{ }^{\circ}\right]$ for 4 . . . . . . . . . . . . . . . . . . . . . . . . 48

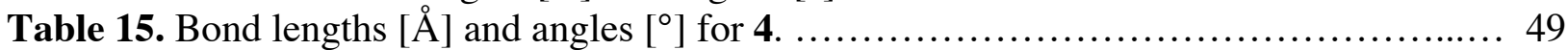

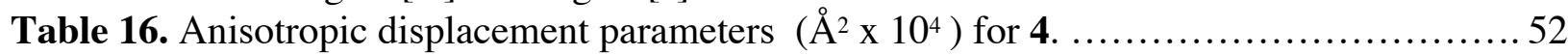

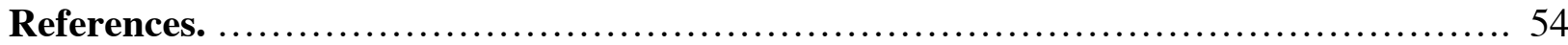




\section{S1. Experimental Section}

\section{General Considerations}

All air- and moisture-sensitive compounds were manipulated using standard vacuum line, Schlenk, or cannula techniques or in a drybox under a nitrogen atmosphere. Solvents for air- and moisture-sensitive reactions were dried over sodium benzophenone ketyl, calcium hydride, or by the method of Grubbs. ${ }^{1}$ Compound $\mathbf{1}$ and $\mathrm{CrPh}_{3}(\mathrm{THF})_{3}$ were prepared as described previously., Dichloromethane- $d_{2}$ was purchased from Cambridge Isotopes and distilled from calcium hydride. Other materials were used as received. Methylaluminumoxane was purchased from Aldrich. UV-Vis measurements were taken on a Hewlett-Packard 8452A diode array spectrometer using a quartz crystal cell. Elemental Analyses were performed by Desert Analytics, Tucson, AZ and by Midwest Microlab, Indianapolis, IN. ${ }^{1} \mathrm{H}$ and ${ }^{13} \mathrm{C}$ NMR spectra were recorded on Varian Mercury 300, or Varian INOVA-500 spectrometers at room temperature, unless indicated otherwise. Chemical shifts are reported with respect to internal solvent: $5.32 \mathrm{ppm}$, and $54.00(\mathrm{t}) \mathrm{ppm}\left(\mathrm{CD}_{2} \mathrm{Cl}_{2}\right)$ for ${ }^{1} \mathrm{H}$ and ${ }^{13} \mathrm{C}$ data. ${ }^{2} \mathrm{H}$ NMR spectra were recorded on a Varian INOVA-500 spectrometer; the chemical shifts are reported with respect to an external $\mathrm{D}_{2} \mathrm{O}$ reference $(4.8 \mathrm{ppm})$.

\section{Synthesis of ( $\left.\mathrm{PNP}^{\mathrm{OMe}}\right) \mathrm{CrCl}_{3}(2)$}

A dichloromethane solution $(20 \mathrm{~mL})$ of $\mathrm{CrCl}_{3}(\mathrm{THF})_{3}(1.513 \mathrm{~g}, 2.9 \mathrm{mmol}, 1$ equiv) was added to a solution of 1 (1.098 g, $2.9 \mathrm{mmol}, 1$ equiv) in dichloromethane ( $30 \mathrm{~mL})$. The color of the reaction mixture turned from purple to blue within 5 min of stirring. The reaction mixture was allowed to stir for $1 \mathrm{~h}$. Volatile materials were removed in vacuo and the blue residue was triturated three times with dichloromethane. The resulting solid was suspended in dichloromethane and stored at $-35{ }^{\circ} \mathrm{C}$ overnight. The desired product was collected as a bright blue powder by filtration through sintered glass frit, washed with dichloromethane, and dried under vacuum. The filtrate contained one or two unidentified paramagnetic species, displaying peaks at 4.85 and $9.5 \mathrm{ppm}$ in the ${ }^{2} \mathrm{H}$ NMR spectrum. Compound 2 obtained in this manner amounted to $1.188 \mathrm{~g}(1.8 \mathrm{mmol}, 60 \%$ yield; this amount corresponds to $53 \%$ yield if the presence of 1 equiv $\mathrm{CH}_{2} \mathrm{Cl}_{2}$ is assumed). X-ray quality crystals were obtained from a dilute 
dichloromethane solution layered with petroleum ether, upon storing at $-35^{\circ} \mathrm{C}$ for days. The crystals submitted to the X-ray diffraction study were prepared via an alternative route, starting from $\left(\mathrm{PNP}^{\mathrm{OMe}}\right) \mathrm{Cr}(\mathrm{CO})_{4}$. Details on this route will be reported in a subsequent paper. ${ }^{2} \mathrm{H} \mathrm{NMR}$ $\left(76 \mathrm{MHz}, \mathrm{CH}_{2} \mathrm{Cl}_{2}\right) \square: 8.8$ (br s, $\left.\mathrm{OCD}_{3}\right)$. $\square_{\text {eff }}=3.8 \square_{B}$. $\square_{\max }\left(\mathrm{CH}_{2} \mathrm{Cl}_{2}, \mathrm{~nm}\right): 536\left(\square=269 \mathrm{M}^{-1} \mathrm{~cm}^{-1}\right)$, $661\left(\left[=484 \mathrm{M}^{-1} \mathrm{~cm}^{-1}\right)\right.$. Anal. calcd. for $\mathrm{C}_{29} \mathrm{H}_{31} \mathrm{Cl}_{3} \mathrm{NO}_{4} \mathrm{P}_{2} \mathrm{Cr}(\%)$ : C, 51.38; H, 4.61; N, 2.07; for $\mathrm{C}_{30} \mathrm{H}_{33} \mathrm{Cl}_{5} \mathrm{NO}_{4} \mathrm{P}_{2} \mathrm{Cr}$ (considering the presence of one equivalent of dichloromethane in the crystal lattice, based on the findings of a single crystal X-ray diffraction study): $\mathrm{C}, 47.23 ; \mathrm{H}, 4.32 ; \mathrm{N}$, 1.84. Found: C, 46.24; H, 4.29; N, 1.77.

\section{Synthesis of $\left(\mathrm{PNP}^{\mathrm{OMe}}\right) \mathrm{CrPh}_{3}(3)$}

In the glove box, compound 1 (1.069 g, $2.01 \mathrm{mmol}, 1.03$ equiv) was dissolved in $75 \mathrm{~mL}$ of dichloromethane. Portions of $\mathrm{CrPh}_{3}(\mathrm{THF})_{3}(974 \mathrm{mg}, 1.95 \mathrm{mmol}$, total, 1 equiv) were added as a slurry in tetrahydrofuran (approximately $2 \mathrm{~mL}$ ) to the stirring solution of 1 over $5 \mathrm{~min}$. The reaction mixture turned a deep red color upon addition. Volatile materials were removed in vacuo, approximately $50 \mathrm{~mL}$ of dichloromethane were added, followed by solvent removal in vасио. The red solid residue was dissolved in approximately $25 \mathrm{~mL}$ of dichloromethane and approximately $30 \mathrm{~mL}$ of petroleum ether were added to precipitate a red solid. This mixture was stored at $-35{ }^{\circ} \mathrm{C}$ overnight. The solid material collected by filtration of the cold solution was recrystallized from a dichloromethane / petroleum ether mixture, collected on a sintered glass frit, and dried under vacuum to leave $1.050 \mathrm{~g}$ of $\mathbf{3}$ as a red, microcrystalline solid (66 \% yield). ${ }^{2} \mathrm{H}$ NMR (RT, $76 \mathrm{MHz}, \mathrm{CH}_{2} \mathrm{Cl}_{2}$ ): $\square 8.5$ ppm (br s, OCD $\mathrm{O}_{3}$ ). Anal. calcd. for $\mathrm{C}_{47} \mathrm{H}_{34} \mathrm{D}_{12} \mathrm{NO}_{4} \mathrm{P}_{2} \mathrm{Cr}$ (\%): C, 69.26; H, 5.70; N, 1.72. Found: C, 69.03; H, 5.69; N, 1.86. $\square_{\text {eff }}=3.8 \square_{B}$. X-ray quality crystals of 3 were obtained from slow diffusion of petroleum ether into a concentrated $\mathrm{CH}_{2} \mathrm{Cl}_{2}$ solution of the complex at $-35{ }^{\circ} \mathrm{C}$.

\section{Synthesis of ( $\left.\mathrm{PNP}^{\mathrm{OMe}}\right) \mathrm{CrBr}\left(o, o^{\prime}\right.$-biphenyldiyl) (4)}

Magnesium turnings (12.3 mg, $1.4 \mathrm{mmol}, 8$ equiv) were added to a diethylether solution (10 $\mathrm{mL})$ of o,o'-dibomobiphenyl (53.5 mg, $0.17 \mathrm{mmol}, 1$ equiv) and allowed to stir overnight at room temperature. The solution was decanted and concentrated to approximately $5 \mathrm{~mL}$ to cause precipitation of a white solid, which was dissolved by addition of dichloromethane. The resulting solution was cooled to almost freezing then added to a thawing dichloromethane 
solution $(20 \mathrm{~mL})$ of $2(0.1183 \mathrm{mg}, 0.17 \mathrm{mmol}, 1$ equiv). The color of the reaction mixture gradually changed from dark blue to forest green upon warming up. The reaction mixture was allowed to stir for $2 \mathrm{~h}$ then volatile materials were removed in vacuo. Dichloromethane was added to the green residue and the mixture filtered through Celite to remove a brown solid. The green filtrate was concentrated, layered with petroleum ether, and cooled to $-35^{\circ} \mathrm{C}$ to cause crystallization of $\mathbf{4}$ as a green material. The green solid was collected on a sintered glass frit, washed with cold dichloromethane and dried under vacuum to provide $104.2 \mathrm{mg}$ of desired product $(0.12 \mathrm{mmol}, 75 \%$ yield). In cases when a white powder (probably magnesium salts) precipitated out along with the desired product, an additional recrystallization provided clean product. X-ray quality crystals were obtained from a dilute dichloromethane solution layered with petroleum ether, upon storing at $-35^{\circ} \mathrm{C}$ for days. ${ }^{2} \mathrm{H} \mathrm{NMR}\left(76 \mathrm{MHz}, \mathrm{CH}_{2} \mathrm{Cl}_{2}\right) \square$ : 5.8 (br s, $\left.\mathrm{OCD}_{3}\right), 9$ (v br s, $\left.\mathrm{OCD}_{3}\right)$. $\square_{\text {eff }}=3.8 \square_{B}$. $\square_{\max }\left(\mathrm{CH}_{2} \mathrm{Cl}_{2}, \mathrm{~nm}\right): 382\left(\square=1937 \mathrm{M}^{-1} \mathrm{~cm}^{-1}\right), 449(\square=809$ $\left.\mathrm{M}^{-1} \mathrm{~cm}^{-1}\right), 598\left(\left[=364 \mathrm{M}^{-1} \mathrm{~cm}^{-1}\right)\right.$. Anal. calcd. for $\mathrm{C}_{41} \mathrm{H}_{39} \mathrm{NO}_{4} \mathrm{P}_{2} \mathrm{BrCr}(\%)$ :, $61.28 ; \mathrm{H}, 4.89 ; \mathrm{N}$, 1.74. Found: C, 61.38; H, 4.93; N, 2.10.

\section{Variable temperature ${ }^{2} \mathrm{H}$ NMR study of 2, 3, and 4}

The experiments were performed with $\mathrm{CH}_{2} \mathrm{Cl}_{2}$ solutions. At high temperatures (fast exchange) the spectra showed one peak. Upon cooling down two decoalescence processes were observed. The higher temperature one splits the peak to two peaks (one broad found between 10-20 ppm and one sharp found in the diamagnetic region $\sim 4 \mathrm{ppm}$ ) in a one to one ratio. The lower temperature decoalescence splits the broad peak mentioned above into two peaks - one broad and shifted downfield ( $>20 \mathrm{ppm}$ ) and one in the diamagnetic region (overlapping with the other diamagnetic peak for $\mathbf{2}$ and $\mathbf{3}$ ). A detailed account of the exchange processes proposed to be the cause of this behavior will be given in a subsequent full paper.

\section{General procedure for trimerization of $\mathrm{C}_{2} \mathrm{H}_{4}$ with 3 activated with $\mathrm{H}\left(\mathrm{Et}_{2} \mathrm{O}\right)_{2} \mathrm{~B}\left[\mathrm{C}_{6} \mathrm{H}_{3}\left(\mathrm{CF}_{3}\right)_{2}\right]_{4}$}

In the glove box a $250 \mathrm{~mL}$ round bottom flask was charged with 3 (16 mg, $20 \square \mathrm{mol}, 1$ equiv) and $\mathrm{H}\left(\mathrm{Et}_{2} \mathrm{O}\right)_{2} \mathrm{~B}\left[\mathrm{C}_{6} \mathrm{H}_{3}\left(\mathrm{CF}_{3}\right)_{2}\right]_{4}(20 \mathrm{mg}, 20$ mol, 1 equiv), and $50 \mathrm{~mL}$ of toluene was added to give a pale green solution. The flask was equipped with a $180^{\circ}$ needle valve, degassed on the vacuum line at $-78^{\circ} \mathrm{C}$, warmed to room temperature, and backfilled with 1 atmosphere of ethylene. After $1 \mathrm{~h}$ the reaction was quenched with $\mathrm{H}_{2} \mathrm{O}$. The organic fraction was separated and filtered 
through a plug of activated alumina to remove any chromium, and this mixture was analyzed by GC-MS. The reaction produces 1-hexene with a range of $700-2,000$ turnovers in greater than $85 \%$ overall selectivity. 


\section{Trimerization of a $\mathrm{C}_{2} \mathrm{D}_{4} / \mathrm{C}_{2} \mathrm{H}_{4}$ mixture with 3 activated with $\mathrm{H}\left(\mathrm{Et}_{2} \mathrm{O}\right)_{2} \mathrm{~B}\left[\mathrm{C}_{6} \mathrm{H}_{3}\left(\mathrm{CF}_{3}\right)_{2}\right]_{4}$}

Chlorobenzene was vacuum transferred into a Schlenk tube charged with 3 (18 mg, 22 पmol, 1 equiv) and $\mathrm{H}\left(\mathrm{Et}_{2} \mathrm{O}\right)_{2} \mathrm{~B}\left[\mathrm{C}_{6} \mathrm{H}_{3}\left(\mathrm{CF}_{3}\right)_{2}\right]_{4}\left(22 \mathrm{mg}, 22 \square \mathrm{mol}, 1\right.$ equiv). A 1:1 mixture of $\mathrm{C}_{2} \mathrm{D}_{4}$ and $\mathrm{C}_{2} \mathrm{H}_{4}$ (128.2 $\mathrm{mL}$ at 213 torr, $1480 \square \mathrm{mol}, 67$ equiv) was condensed into the tube at $-192{ }^{\circ} \mathrm{C}(\sim 1.8$ atm at room temperature). The reaction mixture was allowed to warm to room temperature and left to stir during which time the mixture changed from a red-brown color to a green-brown color. After stirring for 1.5 hours at room temperature, the reaction was vented and quenched with $\mathrm{H}_{2} \mathrm{O}$. The organic fraction was separated and filtered through a plug of activated alumina to remove any chromium, and this mixture was analyzed by GC-MS. The 1-hexene fraction resolves in a quartet showing a 1:3:3:1 distribution of isotopomers $\left(\mathrm{C}_{6} \mathrm{H}_{12}, \mathrm{C}_{6} \mathrm{H}_{8} \mathrm{D}_{4}, \mathrm{C}_{6} \mathrm{H}_{4} \mathrm{D}_{8}\right.$, and $\left.\mathrm{C}_{6} \mathrm{D}_{12}\right)$.

\section{Trimerization of a $\mathrm{C}_{2} \mathrm{D}_{4} / \mathrm{C}_{2} \mathrm{H}_{4}$ mixture with 4 activated with $\mathrm{NaB}\left[\mathrm{C}_{6} \mathrm{H}_{3}\left(\mathrm{CF}_{3}\right)_{2}\right]_{4}$}

$D_{2}$-dichloromethane was vacuum transferred to a J-Young tube charged with 4 (9.2 $\mathrm{mg}, 11.4$ $\square$ mol, 1 equiv) and $\mathrm{NaB}\left[\mathrm{C}_{6} \mathrm{H}_{3}\left(\mathrm{CF}_{3}\right)_{2}\right]_{4}(12.2 \mathrm{mg}, 13.8 \square \mathrm{mol}, 1.2$ equiv). The mixture was warmed up to room temperature using a water bath, followed by mixing (via mechanical rotation) for $10 \mathrm{~min}$. The mixture turned brown upon starting materials dissolving. A 1:1 mixture of $\mathrm{C}_{2} \mathrm{D}_{4}$ and $\mathrm{C}_{2} \mathrm{H}_{4}(128.2 \mathrm{~mL}$ at 29 torr, $200 \square \mathrm{mol}, 17.5$ equiv) was condensed in $(\sim 2.4$ atm in the tube at room temperature). The reaction mixture was allowed to mix for $1 \mathrm{~h}$ at room temperature during which time it achieved a brown green color. The reaction vessel was cooled in a dry ice / acetone bath and degassed. Following removal of ethylene, the mixture was allowed to reach room temperature and volatile materials were vacuum transferred to a round bottom flask and analyzed by GC-MS. The 1-hexene fraction shows a 1:3:3:1 distribution of isotopomers $\left(\mathrm{C}_{6} \mathrm{H}_{12}, \mathrm{C}_{6} \mathrm{H}_{8} \mathrm{D}_{4}, \mathrm{C}_{6} \mathrm{H}_{4} \mathrm{D}_{8}\right.$, and $\left.\mathrm{C}_{6} \mathrm{D}_{12}\right)$. The solid residue was partitioned between water and dichloromethane and the organic fraction was analyzed by GC-MS to reveal the presence of $d_{0^{-}}$and $d_{4}-o$-vinylbiphenyl and biphenyl.

\section{Trimerization of a $\mathrm{C}_{2} \mathrm{D}_{4} / \mathrm{C}_{2} \mathrm{H}_{4}$ mixture with 4 activated with MAO}

Compound 4 (8.2 mg, 10.1 mol, 1 equiv) was suspended in toluene $(30 \mathrm{~mL})$ and the mixture was cooled to $-78{ }^{\circ} \mathrm{C}$ in a dry ice / acetone bath. Under counterflow of argon, the Teflon stopcock was replaced with a rubber septum, and MAO solution (10 \% in toluene, $\mathrm{d}=0.875 \mathrm{~g}$ / $\mathrm{mL}, 2.4 \mathrm{~mL}, 300$ equiv) was added via syringe. The septum was replaced with the Teflon 
stopcock. The mixture was degassed briefly and placed under 1:1 $\mathrm{C}_{2} \mathrm{D}_{4} / \mathrm{C}_{2} \mathrm{H}_{4}$ mixture $(\sim 2.3$ atm static pressure, $10 \mathrm{mmol}, 1000$ equiv) while warming up to room temperature in a water bath. The reaction mixture was allowed to stir for $1.5 \mathrm{~h}$. An aliquot was collected, quenched with water and analyzed by GC-MS. The 1-hexene fraction resolves in a quartet showing a 1:3:3:1 distribution of isotopomers $\left(\mathrm{C}_{6} \mathrm{H}_{12}, \mathrm{C}_{6} \mathrm{H}_{8} \mathrm{D}_{4}, \mathrm{C}_{6} \mathrm{H}_{4} \mathrm{D}_{8}\right.$, and $\left.\mathrm{C}_{6} \mathrm{D}_{12}\right)$. D $0^{-}$and $d_{4}-O$ vinylbiphenyl do not resolve on the GC trace but are both present according to the mass spectrum.

\section{Trimerization of a $\mathrm{C}_{2} \mathrm{D}_{4} / \mathrm{C}_{2} \mathrm{H}_{4}$ mixture with $\mathrm{CrCl}_{3}(\mathrm{THF})_{3} / \mathrm{PNP}^{\mathrm{OMe}}$ (1) activated with MAO}

A solution of $\mathrm{CrCl}_{3}(\mathrm{THF})_{3}(6 \mathrm{mg}, 16 \square \mathrm{mol}, 1$ equiv) in dichloromethane $(1 \mathrm{~mL})$ was added to a dichloromethane solution $(1 \mathrm{~mL})$ of $\mathbf{1}(8.3 \mathrm{mg}, 16 \square \mathrm{mol}, 1$ equiv). The reaction mixture was allowed to stir for $1 \mathrm{~h}$ at room temperature. Volatile materials were removed in vacuo, and toluene $(40 \mathrm{ml})$ was added. The resulting mixture was cooled down in a dry ice / acetone bath and the Teflon stopcock was replaced with a rubber septum under counterflow of argon. MAO solution ( $10 \%$ in toluene, $\mathrm{d}=0.875 \mathrm{~g} / \mathrm{mL}, 3.2 \mathrm{~mL}, 300$ equiv) was added via syringe then the septum was replaced with the Teflon stopcock. The mixture was degassed briefly and placed under 1:1 $\mathrm{C}_{2} \mathrm{D}_{4} / \mathrm{C}_{2} \mathrm{H}_{4}$ mixture $(\sim 1.4 \mathrm{~atm}$ static pressure at room temperature, $1.4 \mathrm{mmol}, 875$ equiv) while warming up to room temperature in a water bath. It was allowed to stir for $2.5 \mathrm{~h}$; the mixture turned pale green. An aliquot was collected, quenched with water and analyzed by GC-MS. The 1-hexene fraction displays a 1:3:3:1 distribution of isotopomers $\left(\mathrm{C}_{6} \mathrm{H}_{12}, \mathrm{C}_{6} \mathrm{H}_{8} \mathrm{D}_{4}\right.$, $\mathrm{C}_{6} \mathrm{H}_{4} \mathrm{D}_{8}$, and $\left.\mathrm{C}_{6} \mathrm{D}_{12}\right)$.

\section{Reaction of 4 with ethylene}

$D_{2}$-dichloromethane was vacuum transferred to a J-Young tube charged with 4 (8.1 mg, 10.1 $\square \mathrm{mol}, 1$ equiv). Ethylene (43.48 mL at 87 torr, $200 \square \mathrm{mol}, 20$ equiv) was condensed in ( $2 \mathrm{~atm}$ in the tube at room temperature). The mixture was warmed up to room temperature using a water bath then allowed to mix by mechanical rotation for $1 \mathrm{~h}$. During this time the mixture achieved a brown-green color. $O$-vinyl-biphenyl was detected by ${ }^{1} \mathrm{H}$ NMR spectroscopy, but no 1-hexene was observed. After an additional $1 \mathrm{~h}$ of mixing the mixture turned brown-red, but no 1-hexene was formed according to ${ }^{1} \mathrm{H}$ NMR spectroscopy. 


\section{Trimerization of cis-, trans-, and gem- $\mathrm{C}_{2} \mathrm{H}_{2} \mathrm{D}_{2}$}

$D_{2}$-dichloromethane was vacuum transferred to a J-Young tube or Schlenk flask charged with 4

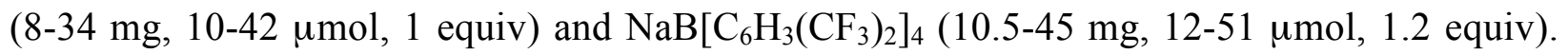
The mixture was warmed up to room temperature using a water bath followed by mixing (via mechanical rotation for NMR tubes or magnetic stirring for flasks) for $10 \mathrm{~min}$. The mixture turned brown upon starting materials dissolving. Labeled ethylene (128.2 mL at 30-125 torr, 200-860 $\square \mathrm{mol}, 17.5-23$ equiv) was condensed in ( 2.3-3.8 atm in the vessel at room temperature). The reaction mixture was allowed to mix for 1-1.5 $\mathrm{h}$ at room temperature during which the mixture turned brown-green. Subsequently, the reaction mixture was cooled in a dry ice / acetone bath and degassed. Following removal of ethylene, the mixture was allowed to warm to room temperature, and volatile materials were vacuum transferred to a round bottom flask and analyzed by ${ }^{1} \mathrm{H}$ and ${ }^{13} \mathrm{C}$ NMR spectroscopy. The solid residue was partitioned between water and dichloromethane and the organic fraction was analyzed by GC-MS to reveal the presence of $d_{2}$ - $O$-vinylbiphenyl and biphenyl. ${ }^{13} \mathrm{C}$ NMR of 1 -hexenes resulted from the trimerization of trans $-\mathrm{C}_{2} \mathrm{H}_{2} \mathrm{D}_{2}\left(126 \mathrm{MHz}, \mathrm{CD}_{2} \mathrm{Cl}_{2}\right) \square: 139.8(\mathrm{~s},=C \mathrm{H}) 139.4(\mathrm{t},=C \mathrm{D}), 114.1$ and $114.0(\mathrm{t}, \mathrm{RHC}=C \mathrm{HD}$ and $\mathrm{RDC}=C H \mathrm{HD}), 33.4(\mathrm{t}, C \mathrm{HD}), 31.1(\mathrm{t}, C H D), 22.2(\mathrm{t}, C H D), 13.8(\mathrm{t}$, $\left.\mathrm{CH}_{2} \mathrm{D}\right)$.

The isotope effects for 1-hexene formation were determined from the integrals of the peaks in the olefinic region (see Table 1 for actual values and section S3 for examples). At this time, it is not clear what is the cause of isotope effect variation with the type of vessel used for the reaction. Generation of the six possible double bond $d_{1}$ - and $d_{2}$-isotopomers of 1-hexene (1,2-cis and trans- $d_{2}$ and $h_{2}-1$-hexene and 1,1- $d_{2}$ and $h_{2}-1$-hexene) provided access to their spectroscopic features in the olefinic region of the ${ }^{1} \mathrm{H}$ NMR spectrum (Figure 1, see also section S3). 


\begin{tabular}{|c|c|c|c|c|c|c|}
\hline & \multicolumn{3}{|c|}{ Cis- $\mathrm{C}_{2} \mathrm{H}_{2} \mathrm{D}_{2}$} & Trans- $\mathrm{C}_{2} \mathrm{H}_{2} \mathrm{D}_{2}$ & \multicolumn{2}{|c|}{ Gem- $\mathrm{C}_{2} \mathrm{H}_{2} \mathrm{D}_{2}$} \\
\hline NMR tube (RT) & 3.5 & & 3.1 & 3.4 & 0.9 & 0.9 \\
\hline $10 \mathrm{~mL}$ RB Schlenk flask (RT) & 2.3 & & --- & --- & \multicolumn{2}{|c|}{--- } \\
\hline $\begin{array}{l}5 \mathrm{~mL} \text { Schlenk tube } \\
\text { (Temp - K) }\end{array}$ & $\begin{array}{c}2.4 \\
(298)\end{array}$ & $\begin{array}{c}2.5 \\
(273)\end{array}$ & $\begin{array}{c}3.4 \\
(248)\end{array}$ & --- & \multicolumn{2}{|c|}{1.3} \\
\hline
\end{tabular}

Table 1. Isotope effects for the trimerization of different isomers of dideuteroethylene under various reaction conditions.
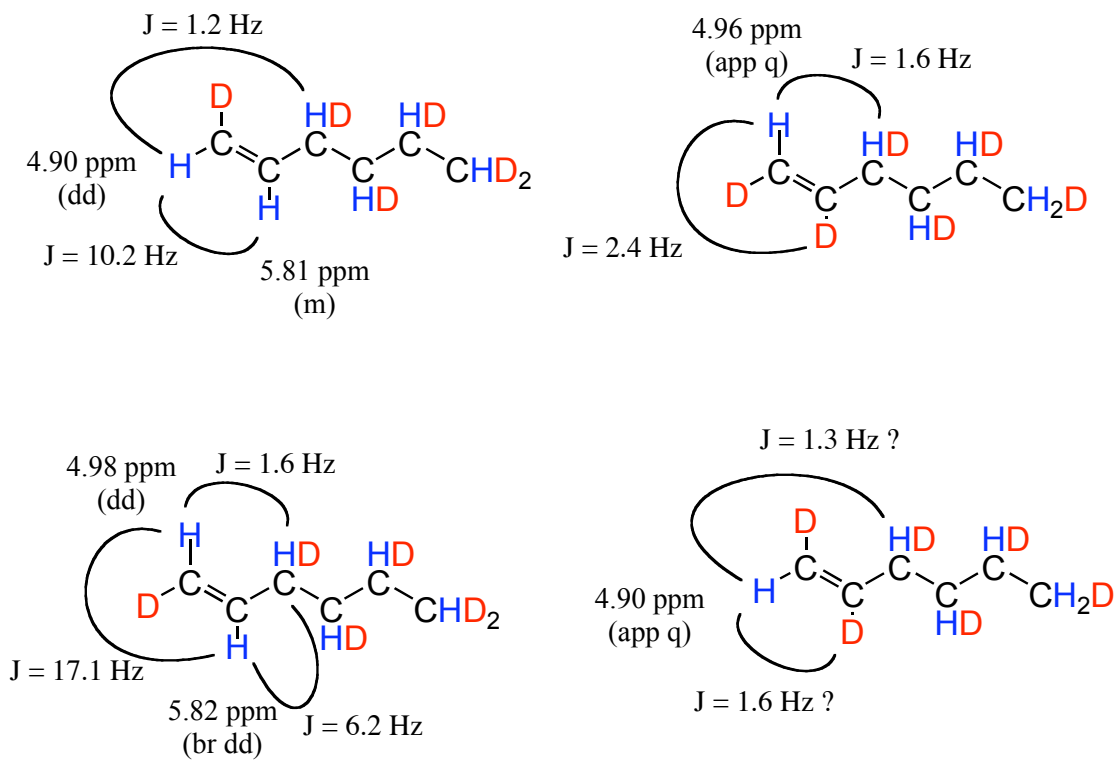<smiles>[2H]C=CCCCCCCCCCC(=O)[O-]</smiles>

5.80-5.83 ppm

(m)<smiles>[2H]CCC(O)CC=CC=CC</smiles>

(m)

Figure 1. ${ }^{1} \mathrm{H} \mathrm{NMR}$ data $\left(\mathrm{CD}_{2} \mathrm{Cl}_{2}, 500 \mathrm{MHz}\right)$ for the olefinic protons of various isotopomers of 1-hexene- $d_{6}$. 
S2. Cossee-type and redox mechanisms for the formation of 1-hexene from ethylene. Expected outcomes and experimental data for the trimerization of a 1:1 mixture of $\mathrm{C}_{2} \mathrm{D}_{4}$ and $\mathrm{C}_{2} \mathrm{H}_{4}$

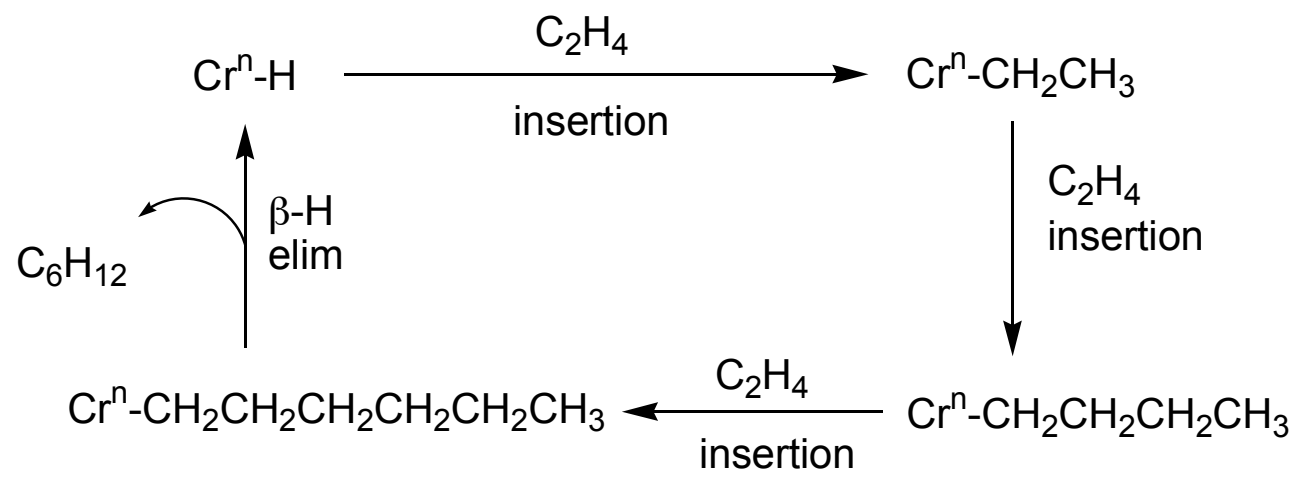

Scheme 1. Proposed Cossee-type mechanism for 1-hexene formation from ethylene.
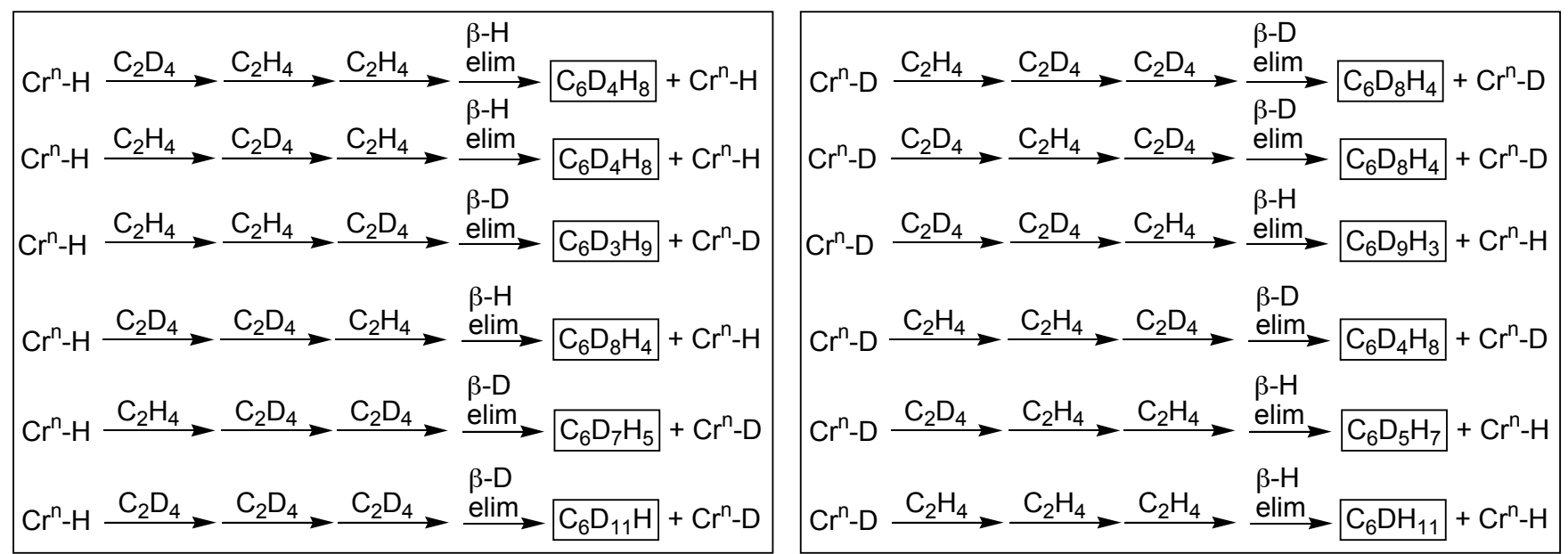

$$
\mathrm{C}_{6} \mathrm{D}_{12}: \mathrm{C}_{6} \mathrm{D}_{11} \mathrm{H}: \mathrm{C}_{6} \mathrm{D}_{9} \mathrm{H}_{3}: \mathrm{C}_{6} \mathrm{D}_{8} \mathrm{H}_{4}: \mathrm{C}_{6} \mathrm{D}_{7} \mathrm{H}_{5}: \mathrm{C}_{6} \mathrm{D}_{5} \mathrm{H}_{7}: \mathrm{C}_{6} \mathrm{D}_{4} \mathrm{H}_{8}: \mathrm{C}_{6} \mathrm{D}_{3} \mathrm{H}_{9}: \mathrm{C}_{6} \mathrm{DH}_{11}: \mathrm{C}_{6} \mathrm{H}_{12}=
$$

Figure 2. Some hexenes that could form from a $\mathrm{C}_{2} \mathrm{D}_{4} / \mathrm{C}_{2} \mathrm{H}_{4} \cdot$ mixture via a Cossee-type mechanism (top). List of all expected isotopomers and their statistical distribution for a Cosseetype mechanism (bottom). 


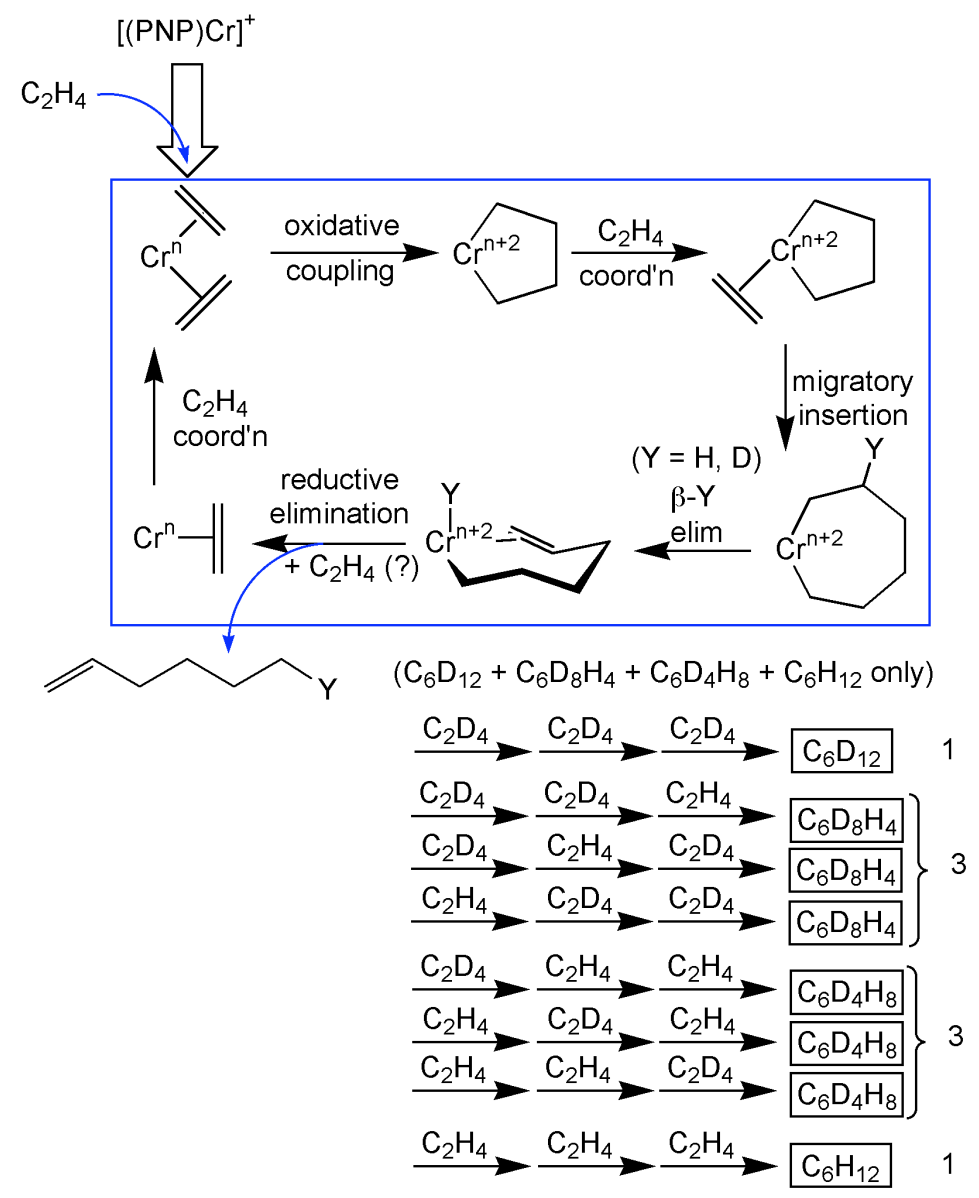

Figure 3. Proposed mechanism for 1-hexene formation from a 1:1 $\mathrm{C}_{2} \mathrm{D}_{4} / \mathrm{C}_{2} \mathrm{H}_{4}$.mixture involving metallacyclic intermediates (top) and expected isotopomer distribution (bottom).

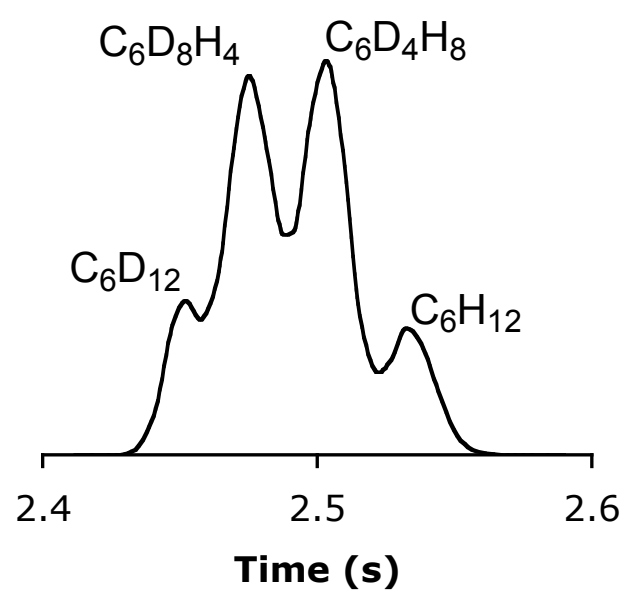

Figure 4. GC data of the 1-hexene fraction obtained from trimerization of a 1:1 mixture of $\mathrm{C}_{2} \mathrm{D}_{4}$ and $\mathrm{C}_{2} \mathrm{H}_{4}$ 


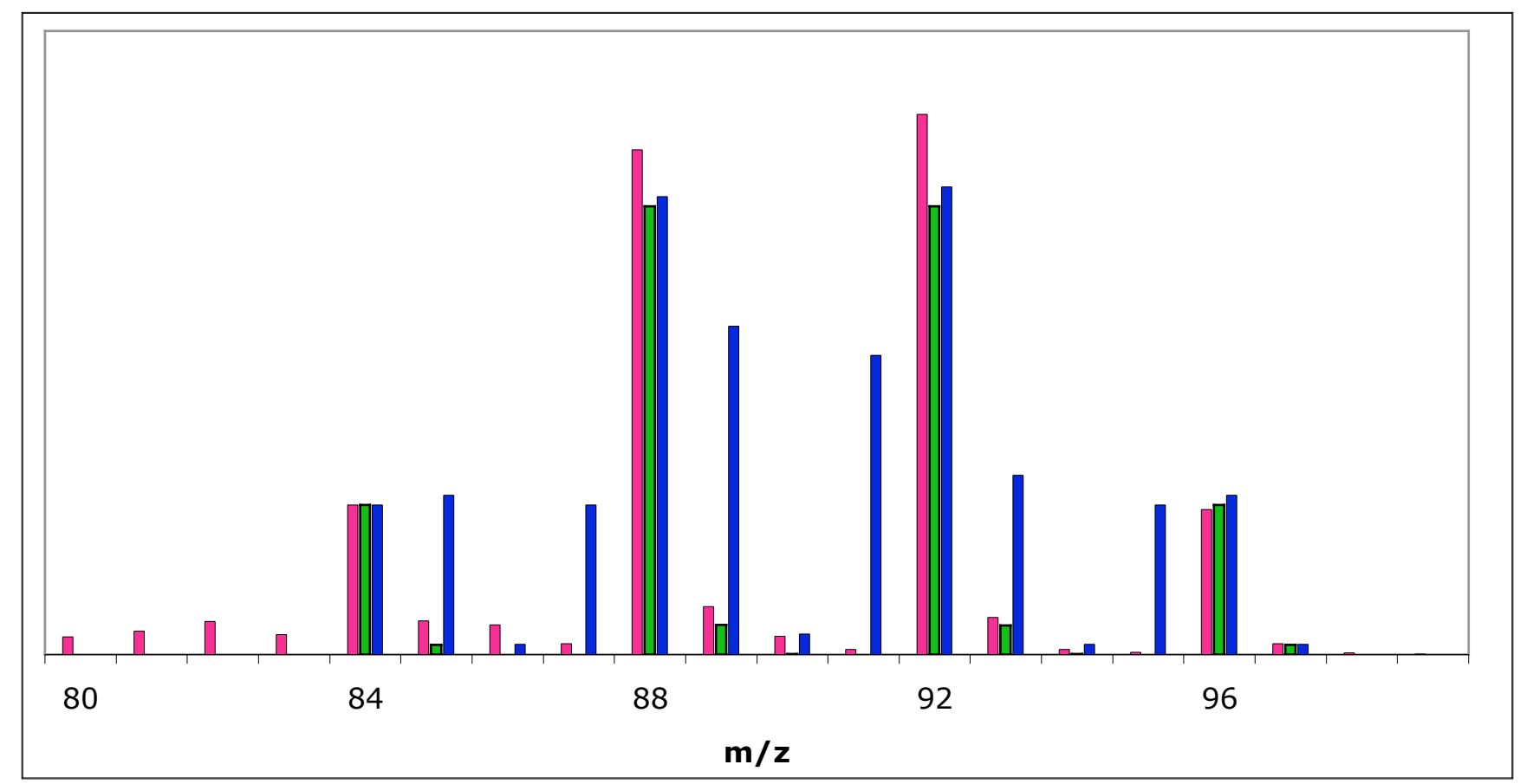

Figure 5. MS data (red) of the 1-hexene fraction obtained from trimerization of a 1:1 mixture of $\mathrm{C}_{2} \mathrm{D}_{4}$ and $\mathrm{C}_{2} \mathrm{H}_{4}$. Simulations of expected isotopomer distributions for a mechanism involving metallacyclic intermediates and for a Cossee-type mechanism are presented in green and blue, respectively. ${ }^{4}$ 
S3. Trimerization of cis-, trans- and gem-dideuteroethylene $-{ }^{l} H$ NMR spectra of the volatile fraction

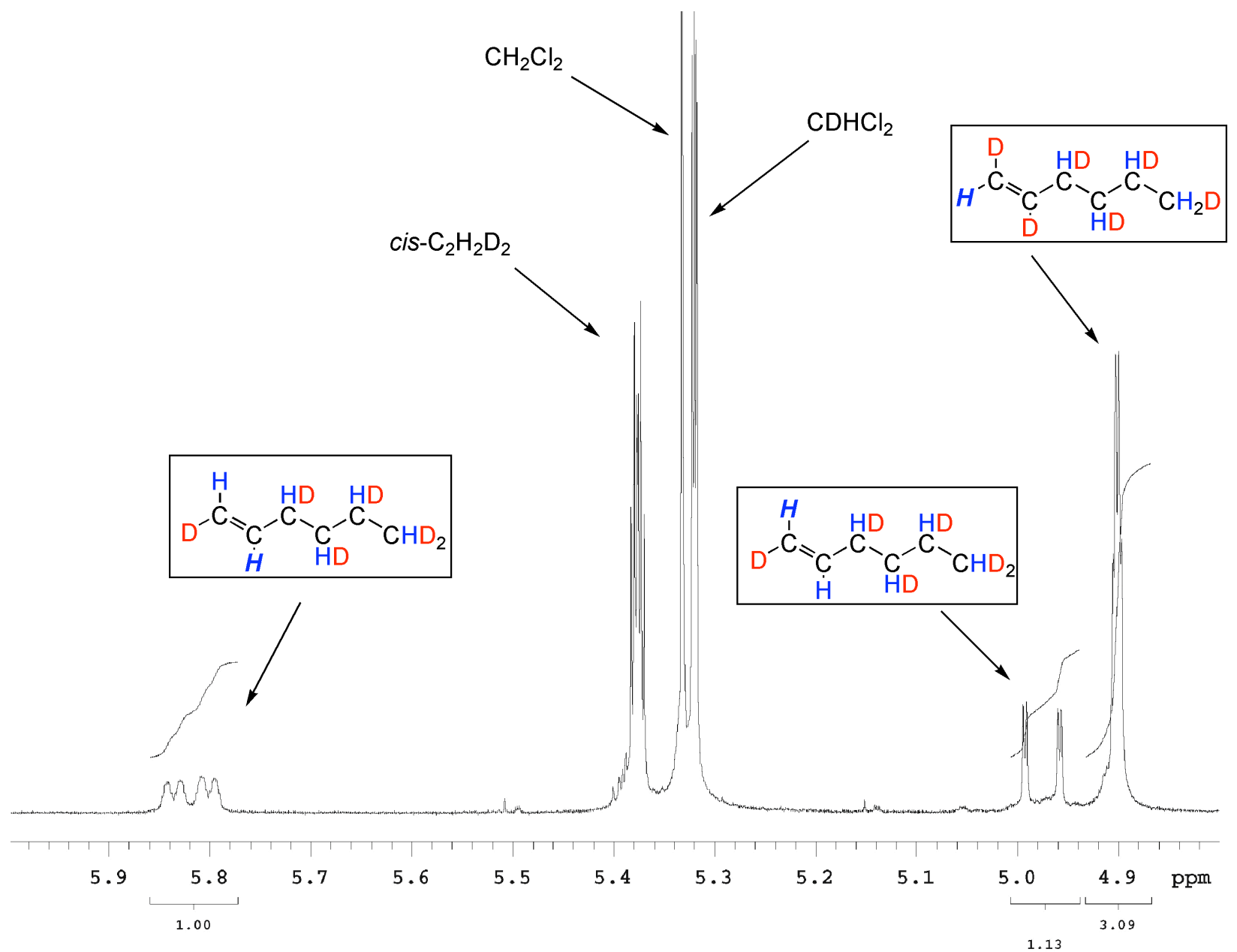

Figure 6. Olefinic region of the ${ }^{1} \mathrm{H}$ NMR spectrum of the volatile materials resulting from the trimerization of cis-dideuteroethylene. 


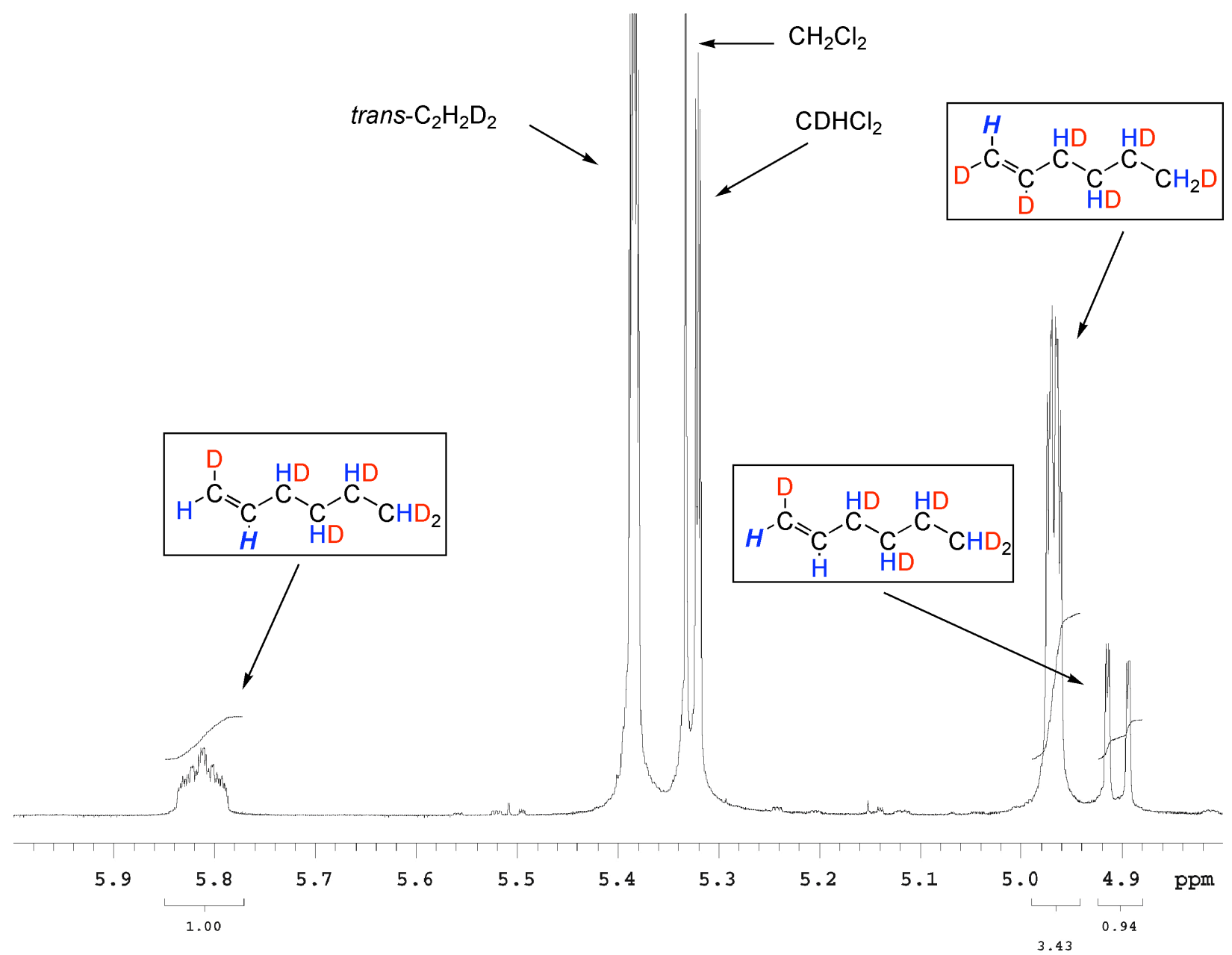

Figure 7. Olefinic region of the ${ }^{1} \mathrm{H}$ NMR spectrum of the volatile materials resulting from the trimerization of trans-dideuteroethylene. 


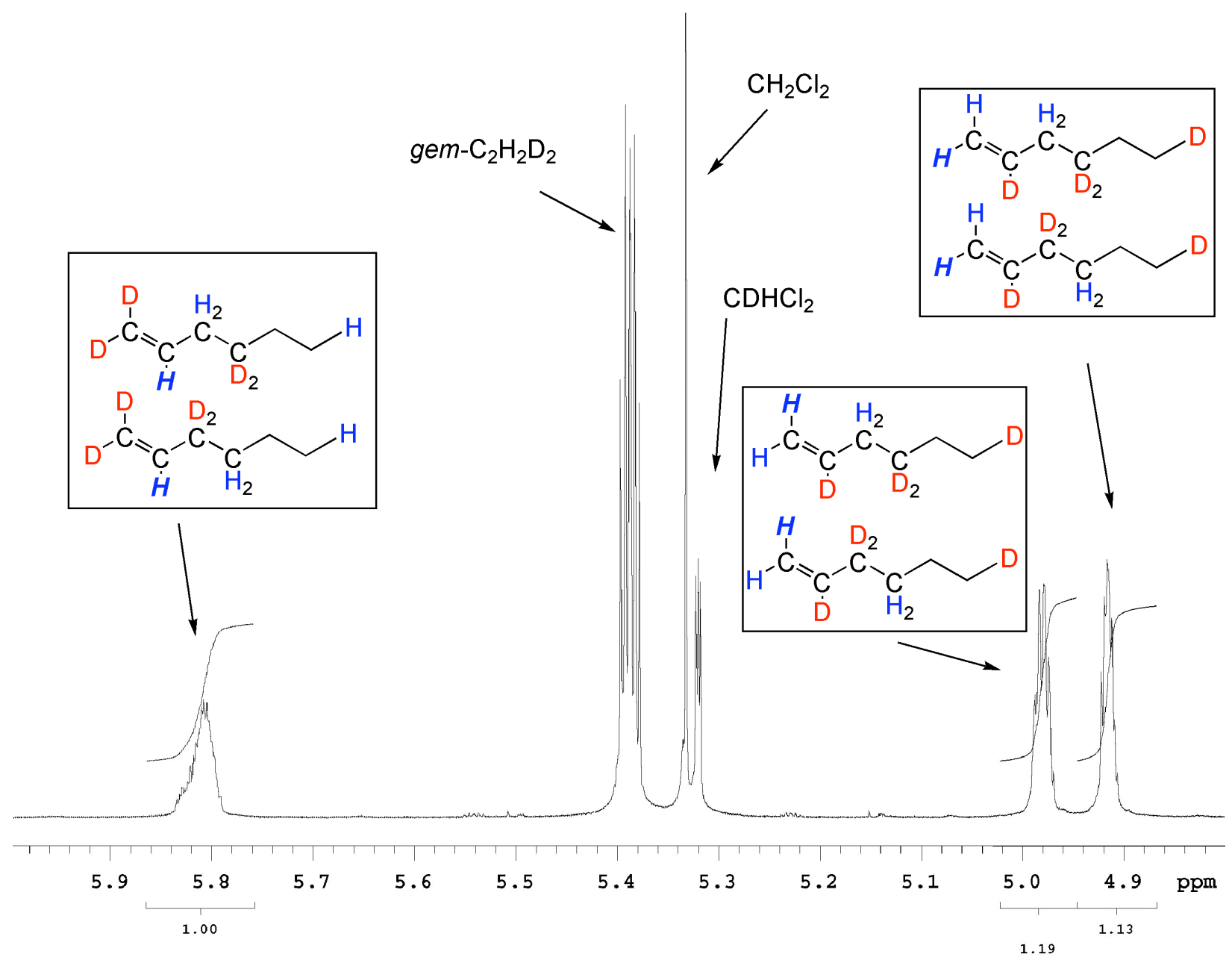

Figure 8. Olefinic region of the ${ }^{1} \mathrm{H}$ NMR spectrum of the volatile materials resulting from the trimerization of gem-dideuteroethylene. 
S4. Expected isotopomeric isomers and isotope effects for the trimerization of cisand gem-dideuteroethylene depending on the mechanism

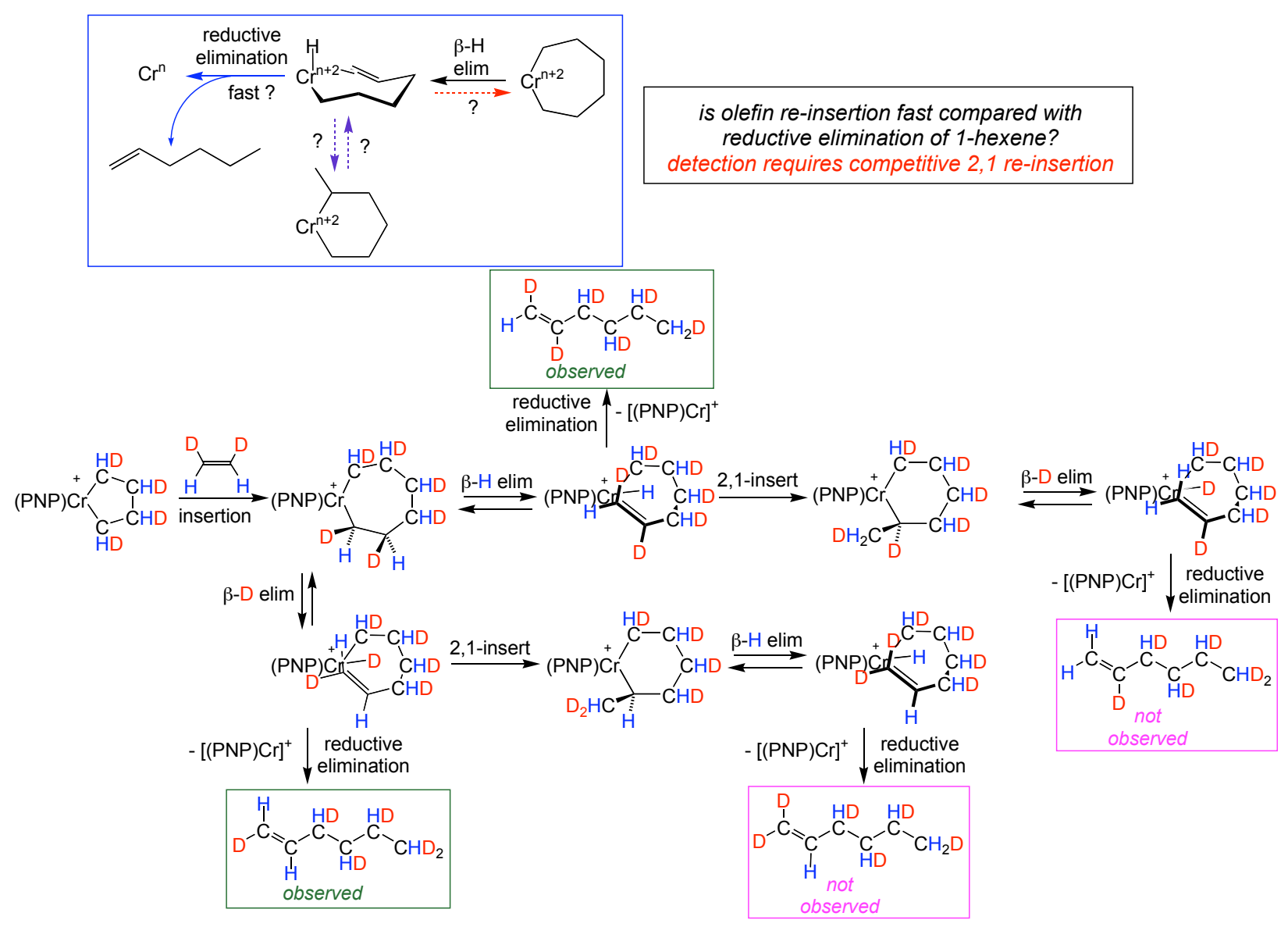

Figure 9. Possible isotopomeric isomers of 1-hexene from mechanism involving 2,1 re-insertion that is competitive with reductive elimination. 

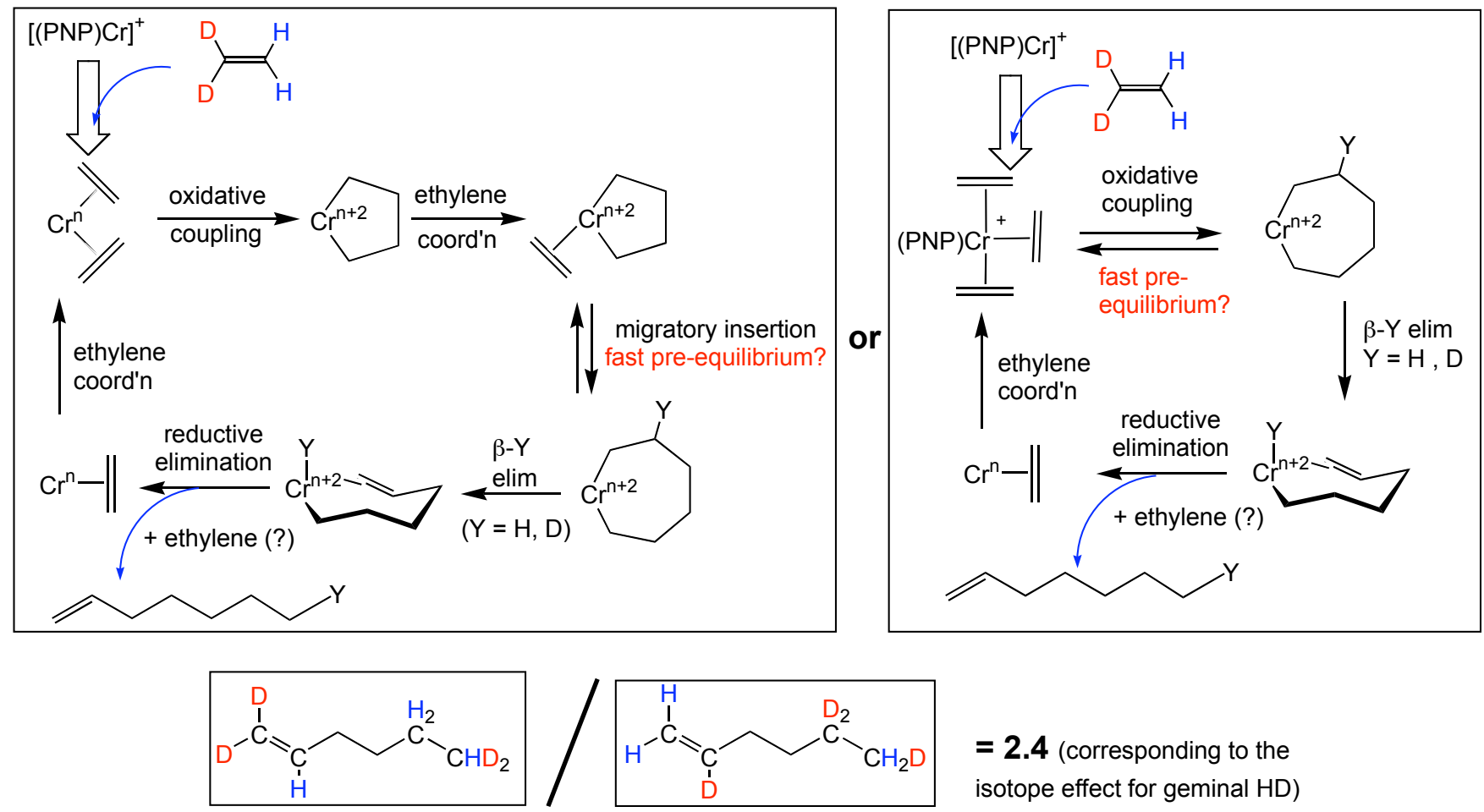

$\mathbf{2} \mathbf{2 . 4}$ (corresponding to the isotope effect for geminal HD)

Figure 10. Proposed mechanisms involving reversible formation of the metallacycloheptane (top). Expected isotope effect for the trimerization of gem-dideuteroethylene would correspond to the one observed for the trimerization of cis- or trans-dideuteroethylene, i. e. coming from the $\checkmark$-hydride abstraction / reductive elimination steps (bottom). 


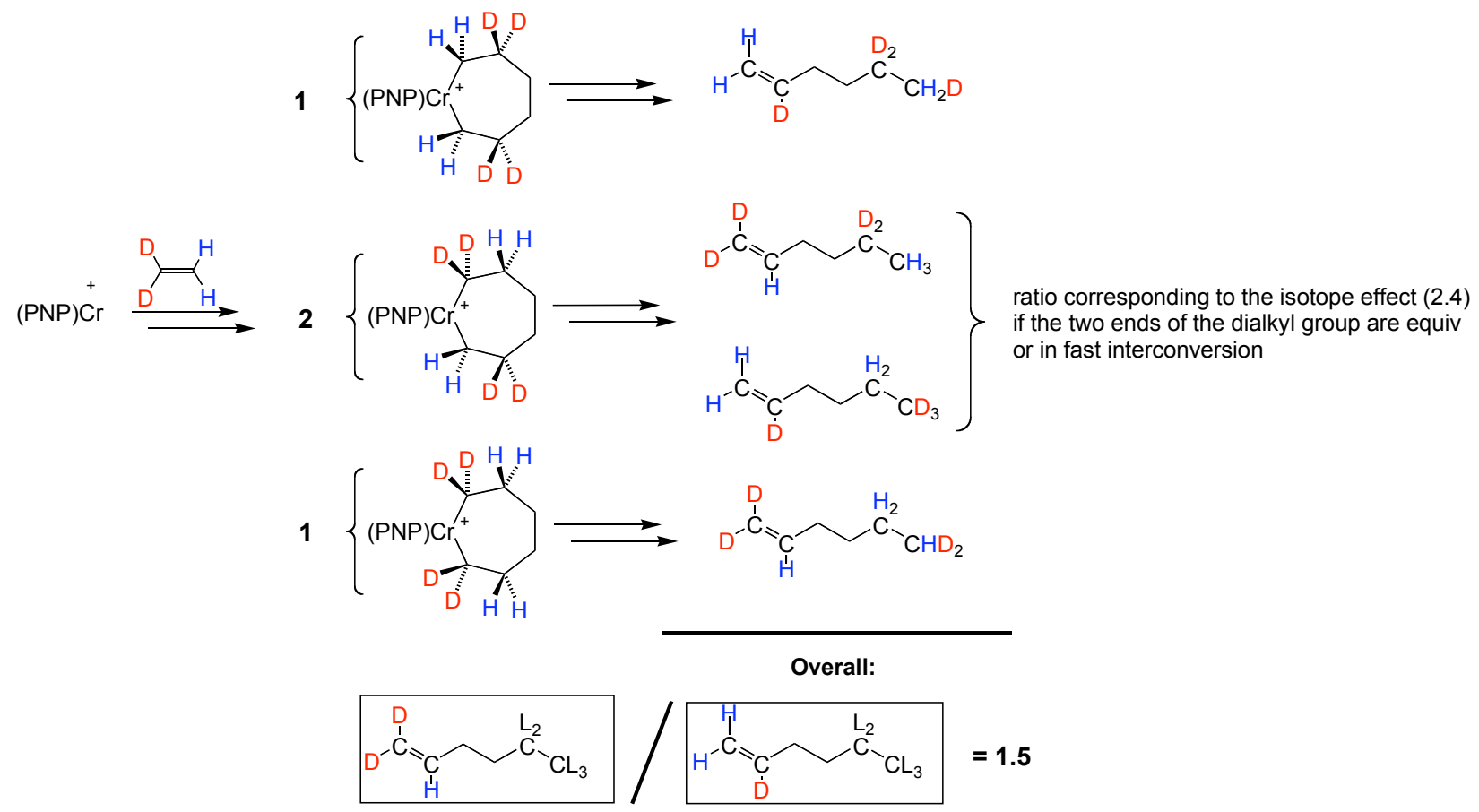

Figure 11. Expected isotopomer distribution from the trimerization of gem-dideuteroethylene for a mechanism involving irreversible formation of the metallacycloheptane and equivalent ends of the alkyl group.

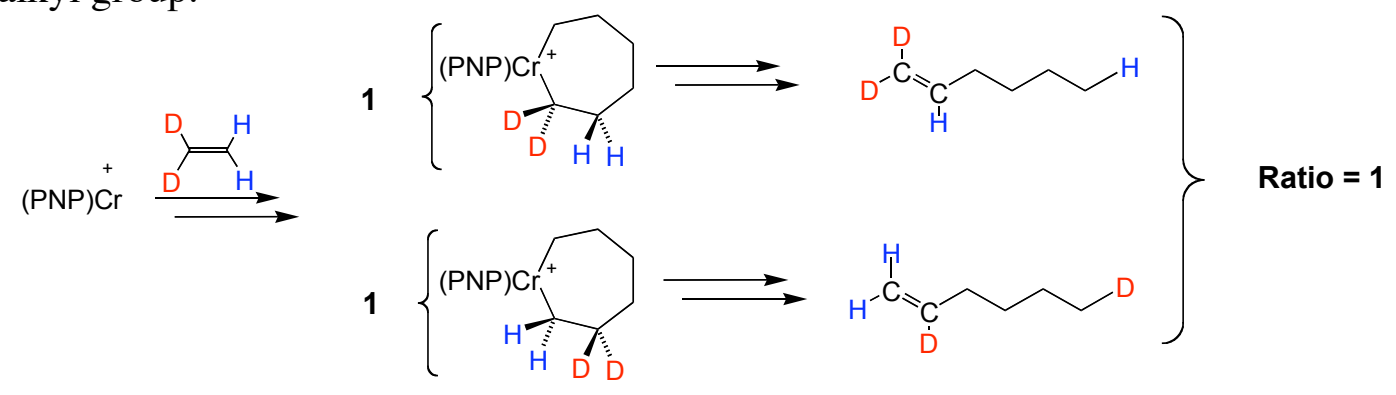

Figure 12. Expected isotopomer distribution from the trimerization of gem-dideuteroethylene for a mechanism involving irreversible formation of the metallacycloheptane and selective $\square$ hydride abstraction / reductive elimination from only one end of the dialkyl group (see Figure 10). 


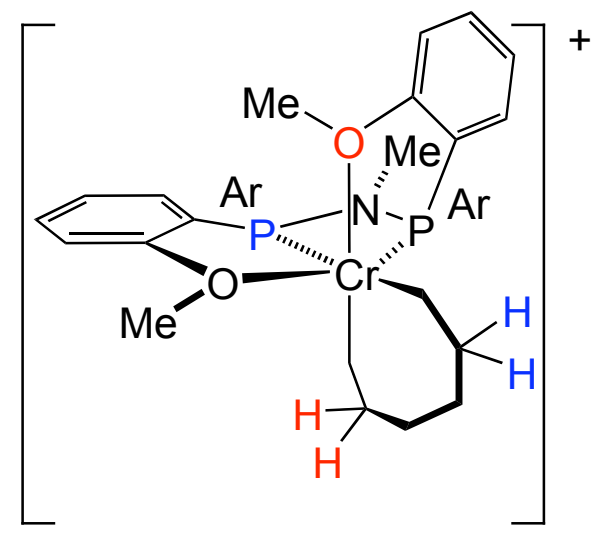

Figure 13. Drawing of the proposed metallacycloheptane intermediate displaying inequivalent ends of the alkanediyl group.

S5. Structural drawings, tables with structural parameters, and crystallographic information for $\mathbf{2}, \mathbf{3}$, and 4

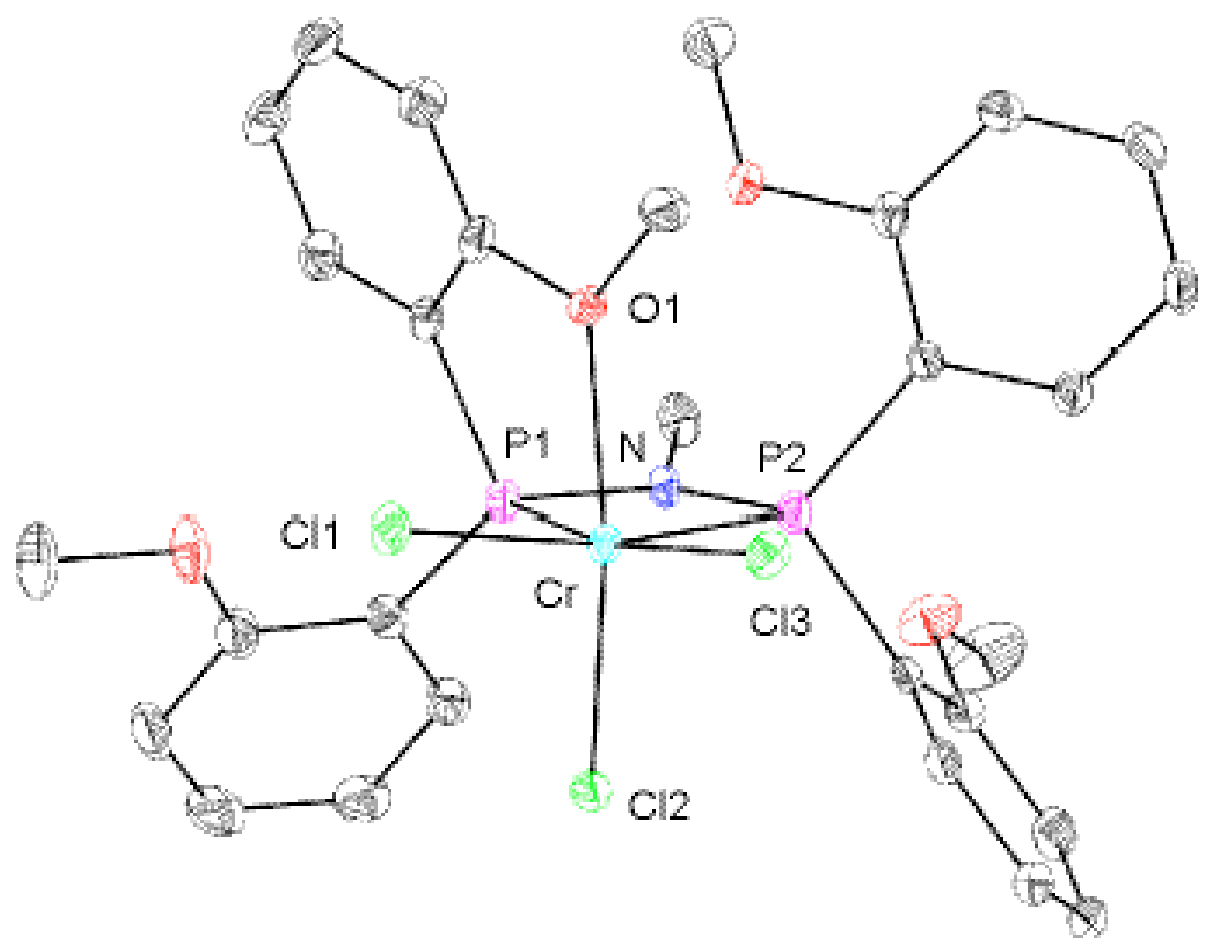

Figure 14. Structural drawing of 2 with thermal ellipsoids at the $50 \%$ probability level. 
Table 2. Crystal data and structure refinement for 2 (CCDC 205652).

Empirical formula

Formula weight

Crystallization Solvent

Crystal Habit

Crystal size

Crystal color

Preliminary Photos

Type of diffractometer

Wavelength

Data Collection Temperature

$\square$ range for 16551 reflections used

in lattice determination

Unit cell dimensions

Volume

Z

Crystal system

Space group

Density (calculated)

$\mathrm{F}(000)$

Data collection program

$\square$ range for data collection

Completeness to $\square=28.17^{\circ}$

Index ranges

Data collection scan type

Data reduction program

Reflections collected

Independent reflections

Absorption coefficient

Absorption correction

Max. and min. transmission
$\mathrm{C}_{29} \mathrm{H}_{31} \mathrm{Cl}_{3} \mathrm{NO}_{4} \mathrm{P}_{2} \mathrm{Cr} \cdot \mathrm{CH}_{2} \mathrm{Cl}_{2}$

762.76

Dichloromethane / petroleum ether

Plate

$0.28 \times 0.27 \times 0.07 \mathrm{~mm}^{3}$

Dark Blue

\section{Data Collection}

Rotation

Bruker SMART 1000

$0.71073 \AA$ MoK $\square$

98(2) K

2.22 to $28.12^{\circ}$

$a=10.6977(7) \AA$

$\mathrm{b}=15.0041(10) \AA$

$c=21.4755(14) \AA$

$\square=96.1720(10)^{\circ}$

3427.0(4) $\AA^{3}$

4

Monoclinic

$\mathrm{P} 2{ }_{1} / \mathrm{n}$

$1.478 \mathrm{Mg} / \mathrm{m}^{3}$

1564

Bruker SMART v5.054

1.66 to $28.17^{\circ}$

$93.1 \%$

$-14 \leq \mathrm{h} \leq 14,-19 \leq \mathrm{k} \leq 18,-28 \leq 1 \leq 28$

$\square$ scans at $5 \square$ settings

Bruker SAINT v6.022

48014

$7838\left[\mathrm{R}_{\mathrm{int}}=0.0696\right]$

$0.853 \mathrm{~mm}^{-1}$

None

0.9427 and 0.7962 
Table 2 (cont.)

\section{Structure solution and Refinement}

Structure solution program

Primary solution method

Secondary solution method

Hydrogen placement

Structure refinement program

Refinement method

Data / restraints / parameters

Treatment of hydrogen atoms

Goodness-of-fit on $\mathrm{F}^{2}$

Final R indices [I $>2 \square(\mathrm{I}), 5783$ reflections]

$\mathrm{R}$ indices (all data)

Type of weighting scheme used

Weighting scheme used

Max shift/error

Average shift/error

Largest diff. peak and hole
SHELXS-97 (Sheldrick, 1990)

Direct methods

Difference Fourier map

Difference Fourier map

SHELXL-97 (Sheldrick, 1997)

Full matrix least-squares on $\mathrm{F}^{2}$

7838 / 0 / 520

Unrestrained

1.579

$\mathrm{R} 1=0.0370, w \mathrm{R} 2=0.0621$

$\mathrm{R} 1=0.0618, w \mathrm{R} 2=0.0649$

Sigma

$w=1 / \square^{2}\left(\mathrm{Fo}^{2}\right)$

0.001

0.000

0.665 and -0.450 e. $\AA^{-3}$

\section{Special Refinement Details}

Refinement of $\mathrm{F}^{2}$ against ALL reflections. The weighted $\mathrm{R}$-factor $(w \mathrm{R})$ and goodness of fit $(\mathrm{S})$ are based on $\mathrm{F}^{2}$, conventional $\mathrm{R}$-factors $(\mathrm{R})$ are based on $\mathrm{F}$, with $\mathrm{F}$ set to zero for negative $\mathrm{F}^{2}$. The threshold expression of $\mathrm{F}^{2}>$ $2 \square\left(\mathrm{F}^{2}\right)$ is used only for calculating $\mathrm{R}$-factors $(\mathrm{gt})$ etc. and is not relevant to the choice of reflections for refinement. $\mathrm{R}$-factors based on $\mathrm{F}^{2}$ are statistically about twice as large as those based on $\mathrm{F}$, and R-factors based on ALL data will be even larger.

All esds (except the esd in the dihedral angle between two 1.s. planes) are estimated using the full covariance matrix. The cell esds are taken into account individually in the estimation of esds in distances, angles and torsion angles; correlations between esds in cell parameters are only used when they are defined by crystal symmetry. An approximate (isotropic) treatment of cell esds is used for estimating esds involving 1.s. planes.

Table 3. Atomic coordinates $\left(x \mathbf{1 0}^{4}\right)$ and equivalent isotropic displacement parameters $\left(\AA^{2} \times 1^{3}\right)$ for 2 (CCDC 205652). U(eq) is defined as the trace of the orthogonalized $U^{i j}$ tensor.

\begin{tabular}{lcccc}
\hline & $\mathrm{x}$ & $\mathrm{y}$ & $\mathrm{z}$ & $\mathrm{U}_{\mathbf{e q}}$ \\
\hline $\mathrm{Cr}(1)$ & $5993(1)$ & $6899(1)$ & $777(1)$ & $12(1)$ \\
$\mathrm{Cl}(1)$ & $5211(1)$ & $7029(1)$ & $-256(1)$ & $17(1)$ \\
$\mathrm{Cl}(2)$ & $7360(1)$ & $8061(1)$ & $760(1)$ & $15(1)$ \\
$\mathrm{Cl}(3)$ & $7455(1)$ & $5779(1)$ & $678(1)$ & $17(1)$ \\
$\mathrm{P}(1)$ & $4517(1)$ & $7857(1)$ & $1199(1)$ & $12(1)$ \\
$\mathrm{P}(2)$ & $6178(1)$ & $6898(1)$ & $1952(1)$ & $12(1)$
\end{tabular}




\begin{tabular}{|c|c|c|c|c|}
\hline $\mathrm{O}(1)$ & $4385(1)$ & $6057(1)$ & $856(1)$ & $14(1)$ \\
\hline $\mathrm{O}(2)$ & $3344(2)$ & $8854(1)$ & $130(1)$ & $25(1)$ \\
\hline $\mathrm{O}(3)$ & $6667(2)$ & 7911(1) & 3180(1) & $25(1)$ \\
\hline $\mathrm{O}(4)$ & 3670(1) & $5966(1)$ & 2133(1) & $17(1)$ \\
\hline $\mathrm{N}(1)$ & 4982(2) & $7640(1)$ & 1962(1) & $12(1)$ \\
\hline $\mathrm{C}(1)$ & $4472(3)$ & $5087(2)$ & $813(1)$ & $20(1)$ \\
\hline $\mathrm{C}(2)$ & $3161(2)$ & $6409(2)$ & 801(1) & $15(1)$ \\
\hline $\mathrm{C}(3)$ & 3046(2) & 7298(2) & 982(1) & $13(1)$ \\
\hline $\mathrm{C}(4)$ & 1854(2) & $7669(2)$ & $958(1)$ & $18(1)$ \\
\hline $\mathrm{C}(5)$ & 798(2) & $7175(2)$ & $762(1)$ & $22(1)$ \\
\hline$C(6)$ & $938(2)$ & $6300(2)$ & $579(1)$ & $22(1)$ \\
\hline$C(7)$ & $2120(2)$ & $5910(2)$ & $585(1)$ & 19(1) \\
\hline $\mathrm{C}(8)$ & $4321(2)$ & $9043(2)$ & $1142(1)$ & $15(1)$ \\
\hline $\mathrm{C}(9)$ & $4830(2)$ & $9588(2)$ & $1629(1)$ & $18(1)$ \\
\hline$C(10)$ & $4769(2)$ & 10511(2) & 1577(1) & $24(1)$ \\
\hline $\mathrm{C}(11)$ & $4209(3)$ & 10881(2) & 1034(1) & $29(1)$ \\
\hline$C(12)$ & 3714(3) & $10366(2)$ & $536(1)$ & $27(1)$ \\
\hline $\mathrm{C}(13)$ & $3771(2)$ & $9435(2)$ & $585(1)$ & $20(1)$ \\
\hline$C(14)$ & 2760(3) & $9220(2)$ & $-447(1)$ & $35(1)$ \\
\hline$C(15)$ & $7647(2)$ & 7332(1) & 2341(1) & $14(1)$ \\
\hline$C(16)$ & $8729(2)$ & 7161(2) & 2057(1) & $17(1)$ \\
\hline $\mathrm{C}(17)$ & $9896(2)$ & 7431(2) & 2331(1) & 19(1) \\
\hline $\mathrm{C}(18)$ & 9989(2) & 7884(2) & 2894(1) & $22(1)$ \\
\hline $\mathrm{C}(19)$ & $8930(2)$ & $8062(2)$ & $3186(1)$ & $22(1)$ \\
\hline$C(20)$ & $7755(2)$ & $7782(2)$ & 2914(1) & $18(1)$ \\
\hline$C(21)$ & $6757(3)$ & $8388(2)$ & $3767(2)$ & $39(1)$ \\
\hline$C(22)$ & $5856(2)$ & $5944(1)$ & $2440(1)$ & $13(1)$ \\
\hline$C(23)$ & $6890(2)$ & $5529(2)$ & 2777(1) & $17(1)$ \\
\hline$C(24)$ & $6740(2)$ & $4812(2)$ & $3167(1)$ & $20(1)$ \\
\hline$C(25)$ & $5548(2)$ & $4518(2)$ & $3250(1)$ & $20(1)$ \\
\hline$C(26)$ & $4507(2)$ & $4908(2)$ & 2920(1) & 19(1) \\
\hline $\mathrm{C}(27)$ & $4656(2)$ & $5597(2)$ & 2501(1) & $15(1)$ \\
\hline $\mathrm{C}(28)$ & $2436(2)$ & $5695(2)$ & $2238(1)$ & $24(1)$ \\
\hline$C(29)$ & $4202(3)$ & $7830(2)$ & $2472(1)$ & $18(1)$ \\
\hline $\mathrm{Cl}(4)$ & $3217(1)$ & $8579(1)$ & $3856(1)$ & $32(1)$ \\
\hline $\mathrm{Cl}(5)$ & $4123(1)$ & $6760(1)$ & 4097(1) & $45(1)$ \\
\hline $\mathrm{C}(31)$ & $3336(3)$ & 7674(2) & $4385(1)$ & $29(1)$ \\
\hline
\end{tabular}


Table 4. Selected bond lengths $[\AA]$ and angles $\left[^{\circ}\right]$ for 2 (CCDC 205652).

\begin{tabular}{lr}
\hline $\mathrm{Cr}(1)-\mathrm{O}(1)$ & $2.1562(15)$ \\
$\mathrm{Cr}(1)-\mathrm{Cl}(2)$ & $2.2776(6)$ \\
$\mathrm{Cr}(1)-\mathrm{Cl}(1)$ & $2.2937(6)$ \\
$\mathrm{Cr}(1)-\mathrm{Cl}(3)$ & $2.3210(7)$ \\
$\mathrm{Cr}(1)-\mathrm{P}(1)$ & $2.3855(7)$ \\
$\mathrm{Cr}(1)-\mathrm{P}(2)$ & $2.5098(7)$ \\
& \\
$\mathrm{O}(1)-\mathrm{Cr}(1)-\mathrm{Cl}(2)$ & $165.62(4)$ \\
$\mathrm{O}(1)-\mathrm{Cr}(1)-\mathrm{Cl}(1)$ & $85.10(4)$ \\
$\mathrm{Cl}(2)-\mathrm{Cr}(1)-\mathrm{Cl}(1)$ & $95.06(2)$ \\
$\mathrm{O}(1)-\mathrm{Cr}(1)-\mathrm{Cl}(3)$ & $97.71(4)$ \\
$\mathrm{Cl}(2)-\mathrm{Cr}(1)-\mathrm{Cl}(3)$ & $96.48(2)$ \\
$\mathrm{Cl}(1)-\mathrm{Cr}(1)-\mathrm{Cl}(3)$ & $98.79(2)$ \\
$\mathrm{O}(1)-\mathrm{Cr}(1)-\mathrm{P}(1)$ & $75.92(4)$ \\
$\mathrm{Cl}(2)-\mathrm{Cr}(1)-\mathrm{P}(1)$ & $89.82(2)$ \\
$\mathrm{Cl}(1)-\mathrm{Cr}(1)-\mathrm{P}(1)$ & $97.39(2)$ \\
$\mathrm{Cl}(3)-\mathrm{Cr}(1)-\mathrm{P}(1)$ & $162.03(3)$ \\
$\mathrm{O}(1)-\mathrm{Cr}(1)-\mathrm{P}(2)$ & $84.11(4)$ \\
$\mathrm{Cl}(2)-\operatorname{Cr}(1)-\mathrm{P}(2)$ & $92.01(2)$ \\
$\mathrm{Cl}(1)-\mathrm{Cr}(1)-\mathrm{P}(2)$ & $162.47(3)$ \\
$\mathrm{Cl}(3)-\mathrm{Cr}(1)-\mathrm{P}(2)$ & $96.31(2)$ \\
$\mathrm{P}(1)-\mathrm{Cr}(1)-\mathrm{P}(2)$ & $66.56(2)$ \\
\end{tabular}


Table 5. Bond lengths $[\AA \AA]$ and angles $\left[^{\circ}\right]$ for 2 (CCDC 205652).

\begin{tabular}{|c|c|c|c|}
\hline $\mathrm{Cr}(1)-\mathrm{O}(1)$ & $2.1562(15)$ & $\mathrm{C}(17)-\mathrm{C}(18)$ & $1.381(3)$ \\
\hline $\mathrm{Cr}(1)-\mathrm{Cl}(2)$ & $2.2776(6)$ & $\mathrm{C}(17)-\mathrm{H}(17)$ & $0.93(2)$ \\
\hline $\mathrm{Cr}(1)-\mathrm{Cl}(1)$ & $2.2937(6)$ & $\mathrm{C}(18)-\mathrm{C}(19)$ & $1.379(4)$ \\
\hline $\mathrm{Cr}(1)-\mathrm{Cl}(3)$ & $2.3210(7)$ & $\mathrm{C}(18)-\mathrm{H}(18)$ & $0.93(2)$ \\
\hline $\mathrm{Cr}(1)-\mathrm{P}(1)$ & $2.3855(7)$ & $\mathrm{C}(19)-\mathrm{C}(20)$ & $1.393(3)$ \\
\hline $\mathrm{Cr}(1)-\mathrm{P}(2)$ & $2.5098(7)$ & C(19)-H(19) & $0.87(2)$ \\
\hline $\mathrm{P}(1)-\mathrm{N}(1)$ & $1.6920(18)$ & $\mathrm{C}(21)-\mathrm{H}(21 \mathrm{~A})$ & $0.97(3)$ \\
\hline $\mathrm{P}(1)-\mathrm{C}(3)$ & $1.800(2)$ & $\mathrm{C}(21)-\mathrm{H}(21 \mathrm{~B})$ & $1.01(3)$ \\
\hline $\mathrm{P}(1)-\mathrm{C}(8)$ & $1.794(2)$ & $\mathrm{C}(21)-\mathrm{H}(21 \mathrm{C})$ & $1.06(3)$ \\
\hline $\mathrm{P}(1)-\mathrm{P}(2)$ & $2.6884(8)$ & $\mathrm{C}(22)-\mathrm{C}(27)$ & $1.404(3)$ \\
\hline $\mathrm{P}(2)-\mathrm{N}(1)$ & $1.6978(18)$ & $\mathrm{C}(22)-\mathrm{C}(23)$ & $1.400(3)$ \\
\hline$P(2)-C(15)$ & $1.820(2)$ & $\mathrm{C}(23)-\mathrm{C}(24)$ & $1.384(3)$ \\
\hline$P(2)-C(22)$ & $1.829(2)$ & $\mathrm{C}(23)-\mathrm{H}(23)$ & $1.00(2)$ \\
\hline $\mathrm{O}(1)-\mathrm{C}(2)$ & $1.404(3)$ & $\mathrm{C}(24)-\mathrm{C}(25)$ & $1.379(3)$ \\
\hline $\mathrm{O}(1)-\mathrm{C}(1)$ & $1.462(3)$ & $\mathrm{C}(24)-\mathrm{H}(24)$ & $0.96(2)$ \\
\hline $\mathrm{O}(2)-\mathrm{C}(13)$ & $1.351(3)$ & $C(25)-C(26)$ & $1.384(3)$ \\
\hline $\mathrm{O}(2)-\mathrm{C}(14)$ & $1.436(3)$ & $\mathrm{C}(25)-\mathrm{H}(25)$ & $0.95(2)$ \\
\hline $\mathrm{O}(3)-\mathrm{C}(20)$ & $1.363(3)$ & $\mathrm{C}(26)-\mathrm{C}(27)$ & $1.391(3)$ \\
\hline $\mathrm{O}(3)-\mathrm{C}(21)$ & $1.444(3)$ & $\mathrm{C}(26)-\mathrm{H}(26)$ & $0.94(2)$ \\
\hline $\mathrm{O}(4)-\mathrm{C}(27)$ & $1.366(2)$ & $\mathrm{C}(28)-\mathrm{H}(28 \mathrm{~A})$ & $0.98(3)$ \\
\hline $\mathrm{O}(4)-\mathrm{C}(28)$ & $1.422(3)$ & $\mathrm{C}(28)-\mathrm{H}(28 \mathrm{~B})$ & $0.98(2)$ \\
\hline $\mathrm{N}(1)-\mathrm{C}(29)$ & $1.475(3)$ & $\mathrm{C}(28)-\mathrm{H}(28 \mathrm{C})$ & $0.96(3)$ \\
\hline $\mathrm{C}(1)-\mathrm{H}(1 \mathrm{~A})$ & $0.97(2)$ & $\mathrm{C}(29)-\mathrm{H}(29 \mathrm{~A})$ & $0.96(3)$ \\
\hline $\mathrm{C}(1)-\mathrm{H}(1 \mathrm{~B})$ & $0.94(2)$ & $\mathrm{C}(29)-\mathrm{H}(29 B)$ & $0.95(2)$ \\
\hline $\mathrm{C}(1)-\mathrm{H}(1 \mathrm{C})$ & $0.99(2)$ & $\mathrm{C}(29)-\mathrm{H}(29 \mathrm{C})$ & $0.97(2)$ \\
\hline $\mathrm{C}(2)-\mathrm{C}(7)$ & $1.379(3)$ & $\mathrm{Cl}(4)-\mathrm{C}(31)$ & $1.767(3)$ \\
\hline$C(2)-C(3)$ & $1.399(3)$ & $\mathrm{Cl}(5)-\mathrm{C}(31)$ & $1.756(3)$ \\
\hline$C(3)-C(4)$ & $1.388(3)$ & $\mathrm{C}(31)-\mathrm{H}(31 \mathrm{~A})$ & $0.88(3)$ \\
\hline$C(4)-C(5)$ & $1.379(3)$ & $\mathrm{C}(31)-\mathrm{H}(31 \mathrm{~B})$ & $0.99(3)$ \\
\hline $\mathrm{C}(4)-\mathrm{H}(4)$ & $0.91(2)$ & & \\
\hline$C(5)-C(6)$ & $1.383(4)$ & $\mathrm{O}(1)-\mathrm{Cr}(1)-\mathrm{Cl}(2)$ & $165.62(4)$ \\
\hline $\mathrm{C}(5)-\mathrm{H}(5)$ & $0.87(2)$ & $\mathrm{O}(1)-\mathrm{Cr}(1)-\mathrm{Cl}(1)$ & $85.10(4)$ \\
\hline$C(6)-C(7)$ & $1.392(3)$ & $\mathrm{Cl}(2)-\mathrm{Cr}(1)-\mathrm{Cl}(1)$ & $95.06(2)$ \\
\hline $\mathrm{C}(6)-\mathrm{H}(6)$ & $0.87(2)$ & $\mathrm{O}(1)-\mathrm{Cr}(1)-\mathrm{Cl}(3)$ & $97.71(4)$ \\
\hline $\mathrm{C}(7)-\mathrm{H}(7)$ & $0.96(2)$ & $\mathrm{Cl}(2)-\mathrm{Cr}(1)-\mathrm{Cl}(3)$ & $96.48(2)$ \\
\hline $\mathrm{C}(8)-\mathrm{C}(13)$ & $1.404(3)$ & $\mathrm{Cl}(1)-\mathrm{Cr}(1)-\mathrm{Cl}(3)$ & $98.79(2)$ \\
\hline $\mathrm{C}(8)-\mathrm{C}(9)$ & $1.392(3)$ & $\mathrm{O}(1)-\mathrm{Cr}(1)-\mathrm{P}(1)$ & $75.92(4)$ \\
\hline $\mathrm{C}(9)-\mathrm{C}(10)$ & $1.391(3)$ & $\mathrm{Cl}(2)-\mathrm{Cr}(1)-\mathrm{P}(1)$ & $89.82(2)$ \\
\hline $\mathrm{C}(9)-\mathrm{H}(9)$ & $0.91(2)$ & $\mathrm{Cl}(1)-\mathrm{Cr}(1)-\mathrm{P}(1)$ & $97.39(2)$ \\
\hline $\mathrm{C}(10)-\mathrm{C}(11)$ & $1.370(4)$ & $\mathrm{Cl}(3)-\mathrm{Cr}(1)-\mathrm{P}(1)$ & $162.03(3)$ \\
\hline $\mathrm{C}(10)-\mathrm{H}(10)$ & $0.92(2)$ & $\mathrm{O}(1)-\mathrm{Cr}(1)-\mathrm{P}(2)$ & $84.11(4)$ \\
\hline $\mathrm{C}(11)-\mathrm{C}(12)$ & $1.377(4)$ & $\mathrm{Cl}(2)-\mathrm{Cr}(1)-\mathrm{P}(2)$ & $92.01(2)$ \\
\hline $\mathrm{C}(11)-\mathrm{H}(11)$ & $0.95(3)$ & $\mathrm{Cl}(1)-\mathrm{Cr}(1)-\mathrm{P}(2)$ & $162.47(3)$ \\
\hline $\mathrm{C}(12)-\mathrm{C}(13)$ & $1.403(3)$ & $\mathrm{Cl}(3)-\mathrm{Cr}(1)-\mathrm{P}(2)$ & $96.31(2)$ \\
\hline $\mathrm{C}(12)-\mathrm{H}(12)$ & $0.90(2)$ & $\mathrm{P}(1)-\mathrm{Cr}(1)-\mathrm{P}(2)$ & $66.56(2)$ \\
\hline $\mathrm{C}(14)-\mathrm{H}(14 \mathrm{~A})$ & $1.04(2)$ & $\mathrm{N}(1)-\mathrm{P}(1)-\mathrm{C}(3)$ & $108.57(9)$ \\
\hline $\mathrm{C}(14)-\mathrm{H}(14 \mathrm{~B})$ & $0.90(2)$ & $\mathrm{N}(1)-\mathrm{P}(1)-\mathrm{C}(8)$ & $106.11(10)$ \\
\hline $\mathrm{C}(14)-\mathrm{H}(14 \mathrm{C})$ & $1.04(3)$ & $\mathrm{C}(3)-\mathrm{P}(1)-\mathrm{C}(8)$ & $110.63(11)$ \\
\hline$C(15)-C(20)$ & $1.398(3)$ & $\mathrm{N}(1)-\mathrm{P}(1)-\mathrm{Cr}(1)$ & $96.53(7)$ \\
\hline$C(15)-C(16)$ & $1.389(3)$ & $\mathrm{C}(3)-\mathrm{P}(1)-\mathrm{Cr}(1)$ & $102.51(7)$ \\
\hline$C(16)-C(17)$ & $1.384(3)$ & $\mathrm{C}(8)-\mathrm{P}(1)-\mathrm{Cr}(1)$ & $130.46(8)$ \\
\hline $\mathrm{C}(16)-\mathrm{H}(16)$ & $0.93(2)$ & $\mathrm{N}(1)-\mathrm{P}(1)-\mathrm{P}(2)$ & $37.60(6)$ \\
\hline
\end{tabular}




\begin{tabular}{|c|c|}
\hline $\mathrm{C}(3)-\mathrm{P}(1)-\mathrm{P}(2)$ & $114.12(7)$ \\
\hline $\mathrm{C}(8)-\mathrm{P}(1)-\mathrm{P}(2)$ & $129.57(8)$ \\
\hline $\mathrm{Cr}(1)-\mathrm{P}(1)-\mathrm{P}(2)$ & $58.93(2)$ \\
\hline $\mathrm{N}(1)-\mathrm{P}(2)-\mathrm{C}(15)$ & $111.99(10)$ \\
\hline $\mathrm{N}(1)-\mathrm{P}(2)-\mathrm{C}(22)$ & $108.51(10)$ \\
\hline $\mathrm{C}(15)-\mathrm{P}(2)-\mathrm{C}(22)$ & $102.89(10)$ \\
\hline $\mathrm{N}(1)-\mathrm{P}(2)-\mathrm{Cr}(1)$ & $91.95(6)$ \\
\hline $\mathrm{C}(15)-\mathrm{P}(2)-\mathrm{Cr}(1)$ & $115.60(8)$ \\
\hline $\mathrm{C}(22)-\mathrm{P}(2)-\mathrm{Cr}(1)$ & $125.32(7)$ \\
\hline $\mathrm{N}(1)-\mathrm{P}(2)-\mathrm{P}(1)$ & $37.45(6)$ \\
\hline $\mathrm{C}(15)-\mathrm{P}(2)-\mathrm{P}(1)$ & $124.53(7)$ \\
\hline $\mathrm{C}(22)-\mathrm{P}(2)-\mathrm{P}(1)$ & $127.68(8)$ \\
\hline $\mathrm{Cr}(1)-\mathrm{P}(2)-\mathrm{P}(1)$ & $54.504(19)$ \\
\hline $\mathrm{C}(2)-\mathrm{O}(1)-\mathrm{C}(1)$ & $115.70(18)$ \\
\hline $\mathrm{C}(2)-\mathrm{O}(1)-\mathrm{Cr}(1)$ & $121.19(13)$ \\
\hline $\mathrm{C}(1)-\mathrm{O}(1)-\mathrm{Cr}(1)$ & $121.45(14)$ \\
\hline $\mathrm{C}(13)-\mathrm{O}(2)-\mathrm{C}(14)$ & $117.3(2)$ \\
\hline $\mathrm{C}(20)-\mathrm{O}(3)-\mathrm{C}(21)$ & 117.1(2) \\
\hline $\mathrm{C}(27)-\mathrm{O}(4)-\mathrm{C}(28)$ & $117.68(19)$ \\
\hline C(29)-N(1)-P(1) & $123.47(15)$ \\
\hline C(29)-N(1)-P(2) & $128.55(15)$ \\
\hline $\mathrm{P}(1)-\mathrm{N}(1)-\mathrm{P}(2)$ & $104.95(10)$ \\
\hline $\mathrm{O}(1)-\mathrm{C}(1)-\mathrm{H}(1 \mathrm{~A})$ & 106.1(14) \\
\hline O(1)-C(1)-H(1B) & $105.5(13)$ \\
\hline $\mathrm{H}(1 \mathrm{~A})-\mathrm{C}(1)-\mathrm{H}(1 \mathrm{~B})$ & $113.5(18)$ \\
\hline $\mathrm{O}(1)-\mathrm{C}(1)-\mathrm{H}(1 \mathrm{C})$ & $108.3(13)$ \\
\hline $\mathrm{H}(1 \mathrm{~A})-\mathrm{C}(1)-\mathrm{H}(1 \mathrm{C})$ & $115.2(19)$ \\
\hline $\mathrm{H}(1 \mathrm{~B})-\mathrm{C}(1)-\mathrm{H}(1 \mathrm{C})$ & $107.7(18)$ \\
\hline $\mathrm{C}(7)-\mathrm{C}(2)-\mathrm{C}(3)$ & $121.2(2)$ \\
\hline $\mathrm{C}(7)-\mathrm{C}(2)-\mathrm{O}(1)$ & $122.4(2)$ \\
\hline $\mathrm{C}(3)-\mathrm{C}(2)-\mathrm{O}(1)$ & $116.4(2)$ \\
\hline$C(4)-C(3)-C(2)$ & $118.8(2)$ \\
\hline $\mathrm{C}(4)-\mathrm{C}(3)-\mathrm{P}(1)$ & $126.61(18)$ \\
\hline $\mathrm{C}(2)-\mathrm{C}(3)-\mathrm{P}(1)$ & $114.60(17)$ \\
\hline$C(3)-C(4)-C(5)$ & $120.9(2)$ \\
\hline $\mathrm{C}(3)-\mathrm{C}(4)-\mathrm{H}(4)$ & $118.9(14)$ \\
\hline $\mathrm{C}(5)-\mathrm{C}(4)-\mathrm{H}(4)$ & $120.2(14)$ \\
\hline$C(6)-C(5)-C(4)$ & 119.1(2) \\
\hline $\mathrm{C}(6)-\mathrm{C}(5)-\mathrm{H}(5)$ & $121.0(16)$ \\
\hline $\mathrm{C}(4)-\mathrm{C}(5)-\mathrm{H}(5)$ & $119.8(16)$ \\
\hline$C(5)-C(6)-C(7)$ & $121.5(2)$ \\
\hline $\mathrm{C}(5)-\mathrm{C}(6)-\mathrm{H}(6)$ & $122.2(15)$ \\
\hline $\mathrm{C}(7)-\mathrm{C}(6)-\mathrm{H}(6)$ & $116.3(15)$ \\
\hline$C(2)-C(7)-C(6)$ & 118.4(2) \\
\hline $\mathrm{C}(2)-\mathrm{C}(7)-\mathrm{H}(7)$ & $121.4(14)$ \\
\hline $\mathrm{C}(6)-\mathrm{C}(7)-\mathrm{H}(7)$ & $120.3(14)$ \\
\hline $\mathrm{C}(13)-\mathrm{C}(8)-\mathrm{C}(9)$ & 119.3(2) \\
\hline $\mathrm{C}(13)-\mathrm{C}(8)-\mathrm{P}(1)$ & $120.57(18)$ \\
\hline C(9)-C(8)-P(1) & $119.85(17)$ \\
\hline$C(8)-C(9)-C(10)$ & $120.9(2)$ \\
\hline $\mathrm{C}(8)-\mathrm{C}(9)-\mathrm{H}(9)$ & $119.9(13)$ \\
\hline $\mathrm{C}(10)-\mathrm{C}(9)-\mathrm{H}(9)$ & $119.2(13)$ \\
\hline $\mathrm{C}(11)-\mathrm{C}(10)-\mathrm{C}(9)$ & $118.9(3)$ \\
\hline $\mathrm{C}(11)-\mathrm{C}(10)-\mathrm{H}(10)$ & $120.1(15)$ \\
\hline
\end{tabular}

$\begin{array}{ll}\mathrm{C}(9)-\mathrm{C}(10)-\mathrm{H}(10) & 120.8(16) \\ \mathrm{C}(10)-\mathrm{C}(11)-\mathrm{C}(12) & 122.0(3) \\ \mathrm{C}(10)-\mathrm{C}(11)-\mathrm{H}(11) & 119.9(16) \\ \mathrm{C}(12)-\mathrm{C}(11)-\mathrm{H}(11) & 117.9(16) \\ \mathrm{C}(11)-\mathrm{C}(12)-\mathrm{C}(13) & 119.4(2) \\ \mathrm{C}(11)-\mathrm{C}(12)-\mathrm{H}(12) & 126.1(16) \\ \mathrm{C}(13)-\mathrm{C}(12)-\mathrm{H}(12) & 114.4(16) \\ \mathrm{O}(2)-\mathrm{C}(13)-\mathrm{C}(8) & 115.1(2) \\ \mathrm{O}(2)-\mathrm{C}(13)-\mathrm{C}(12) & 125.5(2) \\ \mathrm{C}(8)-\mathrm{C}(13)-\mathrm{C}(12) & 119.5(2) \\ \mathrm{O}(2)-\mathrm{C}(14)-\mathrm{H}(14 \mathrm{~A}) & 109.8(13) \\ \mathrm{O}(2)-\mathrm{C}(14)-\mathrm{H}(14 \mathrm{~B}) & 104.7(14) \\ \mathrm{H}(14 \mathrm{~A})-\mathrm{C}(14)-\mathrm{H}(14 \mathrm{~B}) & 115(2) \\ \mathrm{O}(2)-\mathrm{C}(14)-\mathrm{H}(14 \mathrm{C}) & 107.5(14) \\ \mathrm{H}(14 \mathrm{~A})-\mathrm{C}(14)-\mathrm{H}(14 \mathrm{C}) & 114.2(18) \\ \mathrm{H}(14 \mathrm{~B})-\mathrm{C}(14)-\mathrm{H}(14 \mathrm{C}) & 105(2) \\ \mathrm{C}(20)-\mathrm{C}(15)-\mathrm{C}(16) & 118.9(2) \\ \mathrm{C}(20)-\mathrm{C}(15)-\mathrm{P}(2) & 124.23(18) \\ \mathrm{C}(16)-\mathrm{C}(15)-\mathrm{P}(2) & 116.83(18) \\ \mathrm{C}(17)-\mathrm{C}(16)-\mathrm{C}(15) & 121.0(2) \\ \mathrm{C}(17)-\mathrm{C}(16)-\mathrm{H}(16) & 118.4(14) \\ \mathrm{C}(15)-\mathrm{C}(16)-\mathrm{H}(16) & 120.6(14) \\ \mathrm{C}(16)-\mathrm{C}(17)-\mathrm{C}(18) & 119.6(2) \\ \mathrm{C}(16)-\mathrm{C}(17)-\mathrm{H}(17) & 118.5(13) \\ \mathrm{C}(18)-\mathrm{C}(17)-\mathrm{H}(17) & 121.7(13) \\ \mathrm{C}(17)-\mathrm{C}(18)-\mathrm{C}(19) & 120.6(2) \\ \mathrm{C}(17)-\mathrm{C}(18)-\mathrm{H}(18) & 120.2(15) \\ \mathrm{C}(19)-\mathrm{C}(18)-\mathrm{H}(18) & 119.2(15) \\ \mathrm{C}(20)-\mathrm{C}(19)-\mathrm{C}(18) & 120.0(2) \\ \mathrm{C}(20)-\mathrm{C}(19)-\mathrm{H}(19) & 118.9(16) \\ \mathrm{C}(18)-\mathrm{C}(19)-\mathrm{H}(19) & 121.0(16) \\ \mathrm{O}(3)-\mathrm{C}(20)-\mathrm{C}(19) & 123.7(2) \\ \mathrm{O}(3)-\mathrm{C}(20)-\mathrm{C}(15) & 116.3(2) \\ \mathrm{C}(19)-\mathrm{C}(20)-\mathrm{C}(15) & 120.0(2) \\ \mathrm{O}(3)-\mathrm{C}(21)-\mathrm{H}(21 \mathrm{~A}) & 114.4(17) \\ \mathrm{O}(3)-\mathrm{C}(21)-\mathrm{H}(21 \mathrm{~B}) & 108.0(17) \\ \mathrm{H}(21 \mathrm{~A})-\mathrm{C}(21)-\mathrm{H}(21 \mathrm{~B}) & 106(2) \\ \mathrm{O}(3)-\mathrm{C}(21)-\mathrm{H}(21 \mathrm{C}) & 108.6(14) \\ \mathrm{H}(21 \mathrm{~A})-\mathrm{C}(21)-\mathrm{H}(21 \mathrm{C}) & 109(2) \\ \mathrm{H}(21 \mathrm{~B})-\mathrm{C}(21)-\mathrm{H}(21 \mathrm{C}) & 111(2) \\ \mathrm{C}(27)-\mathrm{C}(22)-\mathrm{C}(23) & 117.8(2) \\ \mathrm{C}(27)-\mathrm{C}(22)-\mathrm{P}(2) & 125.00(17) \\ \mathrm{C}(23)-\mathrm{C}(22)-\mathrm{P}(2) & 117.20(17) \\ \mathrm{C}(24)-\mathrm{C}(23)-\mathrm{C}(22) & 121.4(2) \\ \mathrm{C}(24)-\mathrm{C}(23)-\mathrm{H}(23) & 119.6(12) \\ \mathrm{C}(22)-\mathrm{C}(23)-\mathrm{H}(23) & 119.0(12) \\ \mathrm{C}(23)-\mathrm{C}(24)-\mathrm{C}(25) & 119.8(2) \\ \mathrm{C}(23)-\mathrm{C}(24)-\mathrm{H}(24) & 118.8(15) \\ \mathrm{C}(25)-\mathrm{C}(24)-\mathrm{H}(24) & 121.4(15) \\ \mathrm{C}(26)-\mathrm{C}(25)-\mathrm{C}(24) & 120.2(2) \\ \mathrm{C}(26)-\mathrm{C}(25)-\mathrm{H}(25) & 118.8(14) \\ \mathrm{C}(24)-\mathrm{C}(25)-\mathrm{H}(25) & 121.0(14) \\ \mathrm{C}(25)-\mathrm{C}(26)-\mathrm{C}(27) & 118.1(13) \\ \mathrm{C}(25)-\mathrm{C}(26)-\mathrm{H}(26) & \\ & \end{array}$




$\begin{array}{llll}\mathrm{C}(27)-\mathrm{C}(26)-\mathrm{H}(26) & 121.7(13) & \mathrm{N}(1)-\mathrm{C}(29)-\mathrm{H}(29 \mathrm{~B}) & 109.9(14) \\ \mathrm{O}(4)-\mathrm{C}(27)-\mathrm{C}(26) & 122.7(2) & \mathrm{H}(29 \mathrm{~A})-\mathrm{C}(29)-\mathrm{H}(29 \mathrm{~B}) & 111(2) \\ \mathrm{O}(4)-\mathrm{C}(27)-\mathrm{C}(22) & 116.95(19) & \mathrm{N}(1)-\mathrm{C}(29)-\mathrm{H}(29 \mathrm{C}) & 108.2(14) \\ \mathrm{C}(26)-\mathrm{C}(27)-\mathrm{C}(22) & 120.4(2) & \mathrm{H}(29 \mathrm{~A})-\mathrm{C}(29)-\mathrm{H}(29 \mathrm{C}) & 107(2) \\ \mathrm{O}(4)-\mathrm{C}(28)-\mathrm{H}(28 \mathrm{~A}) & 110.0(15) & \mathrm{H}(29 \mathrm{~B})-\mathrm{C}(29)-\mathrm{H}(29 \mathrm{C}) & 108(2) \\ \mathrm{O}(4)-\mathrm{C}(28)-\mathrm{H}(28 \mathrm{~B}) & 105.7(14) & \mathrm{Cl}(5)-\mathrm{C}(31)-\mathrm{Cl}(4) & 112.21(15) \\ \mathrm{H}(28 \mathrm{~A})-\mathrm{C}(28)-\mathrm{H}(28 \mathrm{~B}) & 111(2) & \mathrm{Cl}(5)-\mathrm{C}(31)-\mathrm{H}(31 \mathrm{~A}) & 108.5(18) \\ \mathrm{O}(4)-\mathrm{C}(28)-\mathrm{H}(28 \mathrm{C}) & 110.8(15) & \mathrm{Cl}(4)-\mathrm{C}(31)-\mathrm{H}(31 \mathrm{~A}) & 111.1(18) \\ \mathrm{H}(28 \mathrm{~A})-\mathrm{C}(28)-\mathrm{H}(28 \mathrm{C}) & 109(2) & \mathrm{Cl}(5)-\mathrm{C}(31)-\mathrm{H}(31 \mathrm{~B}) & 113.1(16) \\ \mathrm{H}(28 \mathrm{~B})-\mathrm{C}(28)-\mathrm{H}(28 \mathrm{C}) & 110(2) & \mathrm{Cl}(4)-\mathrm{C}(31)-\mathrm{H}(31 \mathrm{~B}) & 107.9(16) \\ \mathrm{N}(1)-\mathrm{C}(29)-\mathrm{H}(29 \mathrm{~A}) & 112.1(15) & \mathrm{H}(31 \mathrm{~A})-\mathrm{C}(31)-\mathrm{H}(31 \mathrm{~B}) & 104(2)\end{array}$


Table 6. Anisotropic displacement parameters $\left(\AA^{2} \times 10^{4}\right)$ for 2 (CCDC 205652). The anisotropic displacement factor exponent takes the form: $-2 \square^{2}\left[h^{2} a^{* 2} U^{11}+\ldots+2 h k a^{*} b^{*}\right.$ $\left.\mathbf{U}^{12}\right]$

\begin{tabular}{|c|c|c|c|c|c|c|}
\hline & $\mathrm{U}^{11}$ & $\mathrm{U}^{22}$ & $\mathrm{U}^{33}$ & $\mathrm{U}^{23}$ & $\mathrm{U}^{13}$ & $\mathrm{U}^{12}$ \\
\hline $\operatorname{Cr}(1)$ & $129(2)$ & $107(2)$ & $105(2)$ & $-7(2)$ & $-13(1)$ & $3(2)$ \\
\hline $\mathrm{Cl}(1)$ & 198(3) & 192(3) & 111(3) & $-6(2)$ & $-21(2)$ & $-1(3)$ \\
\hline $\mathrm{Cl}(2)$ & $165(3)$ & $136(3)$ & $147(3)$ & $4(2)$ & $-4(2)$ & $-15(2)$ \\
\hline $\mathrm{Cl}(3)$ & $150(3)$ & $143(3)$ & 209(3) & $-33(2)$ & $-4(2)$ & $28(2)$ \\
\hline $\mathrm{P}(1)$ & $142(3)$ & $116(3)$ & 111(3) & $3(2)$ & $-13(2)$ & $14(2)$ \\
\hline $\mathrm{P}(2)$ & 141(3) & $114(3)$ & $109(3)$ & $4(2)$ & $-17(2)$ & 1(3) \\
\hline $\mathrm{O}(1)$ & 130(9) & $106(8)$ & $166(8)$ & $-19(6)$ & $-21(7)$ & $-1(7)$ \\
\hline $\mathrm{O}(2)$ & $323(11)$ & 268(10) & 137(9) & $46(7)$ & $-48(8)$ & $57(8)$ \\
\hline $\mathrm{O}(3)$ & 242(10) & 330(11) & 163(9) & $-117(8)$ & $17(7)$ & $-69(8)$ \\
\hline $\mathrm{O}(4)$ & 118(9) & 192(9) & 183(9) & 49(7) & $-20(7)$ & 4(7) \\
\hline $\mathrm{N}(1)$ & $146(11)$ & $137(10)$ & $82(10)$ & $14(8)$ & $-3(8)$ & $10(8)$ \\
\hline $\mathrm{C}(1)$ & $185(15)$ & 113(13) & 291(16) & $-7(11)$ & $18(12)$ & $15(11)$ \\
\hline$C(2)$ & $129(13)$ & 211(13) & $107(12)$ & $8(10)$ & $-4(9)$ & $29(10)$ \\
\hline $\mathrm{C}(3)$ & $147(13)$ & $160(12)$ & $80(11)$ & $3(9)$ & $-9(9)$ & $12(10)$ \\
\hline $\mathrm{C}(4)$ & 195(14) & $169(14)$ & 171(13) & $-14(10)$ & $-3(10)$ & $39(11)$ \\
\hline $\mathrm{C}(5)$ & 119(13) & $335(16)$ & 199(14) & $-5(11)$ & 10(11) & $52(12)$ \\
\hline$C(6)$ & $157(14)$ & 271(15) & 212(14) & $-14(11)$ & $-33(11)$ & $-41(12)$ \\
\hline$C(7)$ & $188(14)$ & 177(13) & $188(13)$ & $-31(10)$ & $0(10)$ & $-2(11)$ \\
\hline $\mathrm{C}(8)$ & $147(13)$ & 134(12) & $167(12)$ & $12(10)$ & $38(10)$ & $34(10)$ \\
\hline $\mathrm{C}(9)$ & 174(13) & $165(13)$ & $215(14)$ & 14(11) & $25(11)$ & $24(10)$ \\
\hline $\mathrm{C}(10)$ & 202(14) & $165(14)$ & $346(16)$ & $-39(12)$ & $47(12)$ & 2(11) \\
\hline $\mathrm{C}(11)$ & 277(16) & $132(14)$ & 470(19) & $40(13)$ & $60(13)$ & $17(12)$ \\
\hline $\mathrm{C}(12)$ & 261(16) & 264(16) & 291(16) & $149(13)$ & $37(13)$ & $71(12)$ \\
\hline $\mathrm{C}(13)$ & 189(13) & 203(13) & 206(13) & $35(11)$ & 10(11) & $30(11)$ \\
\hline $\mathrm{C}(14)$ & $410(20)$ & $430(20)$ & $175(15)$ & $76(14)$ & $-75(14)$ & $80(17)$ \\
\hline$C(15)$ & $164(13)$ & $119(12)$ & $122(12)$ & $48(9)$ & $-25(10)$ & $-22(10)$ \\
\hline$C(16)$ & 201(14) & $130(12)$ & $152(13)$ & $32(10)$ & $-30(10)$ & $6(10)$ \\
\hline $\mathrm{C}(17)$ & $153(14)$ & 190(13) & 221(14) & $59(10)$ & $-10(11)$ & $-4(11)$ \\
\hline $\mathrm{C}(18)$ & 201(14) & 198(14) & $235(14)$ & $64(11)$ & $-96(11)$ & $-65(11)$ \\
\hline C(19) & 291(15) & 209(13) & $131(13)$ & $2(11)$ & $-53(11)$ & $-34(12)$ \\
\hline$C(20)$ & 191(13) & $164(12)$ & $168(13)$ & $33(10)$ & $-21(10)$ & $-19(10)$ \\
\hline$C(21)$ & $350(20)$ & $560(20)$ & $269(17)$ & $-231(15)$ & $40(15)$ & $-82(17)$ \\
\hline$C(22)$ & $154(12)$ & $109(12)$ & $120(11)$ & 11(9) & $-3(9)$ & $8(10)$ \\
\hline $\mathrm{C}(23)$ & $183(14)$ & $140(12)$ & $167(13)$ & $5(10)$ & $-37(10)$ & $-6(11)$ \\
\hline$C(24)$ & $234(15)$ & $156(13)$ & 199(14) & $37(10)$ & $-63(11)$ & $38(11)$ \\
\hline$C(25)$ & 292(15) & $137(13)$ & $149(13)$ & $40(10)$ & $-15(11)$ & $-40(11)$ \\
\hline$C(26)$ & 202(14) & $167(13)$ & $195(13)$ & $18(10)$ & $3(11)$ & $-45(11)$ \\
\hline $\mathrm{C}(27)$ & $184(13)$ & $121(12)$ & $124(12)$ & $-18(9)$ & $-28(10)$ & $15(10)$ \\
\hline $\mathrm{C}(28)$ & 171(14) & $287(17)$ & $277(16)$ & $61(13)$ & $36(12)$ & $3(12)$ \\
\hline$C(29)$ & $230(15)$ & 192(14) & $130(13)$ & $12(11)$ & $34(11)$ & $49(11)$ \\
\hline $\mathrm{Cl}(4)$ & 349(4) & $345(4)$ & 271(4) & $48(3)$ & $80(3)$ & $122(3)$ \\
\hline $\mathrm{Cl}(5)$ & $503(5)$ & $328(4)$ & $548(5)$ & $135(4)$ & $226(4)$ & $104(4)$ \\
\hline $\mathrm{C}(31)$ & $317(18)$ & $372(17)$ & 192(16) & $11(13)$ & 43(13) & $-3(14)$ \\
\hline
\end{tabular}




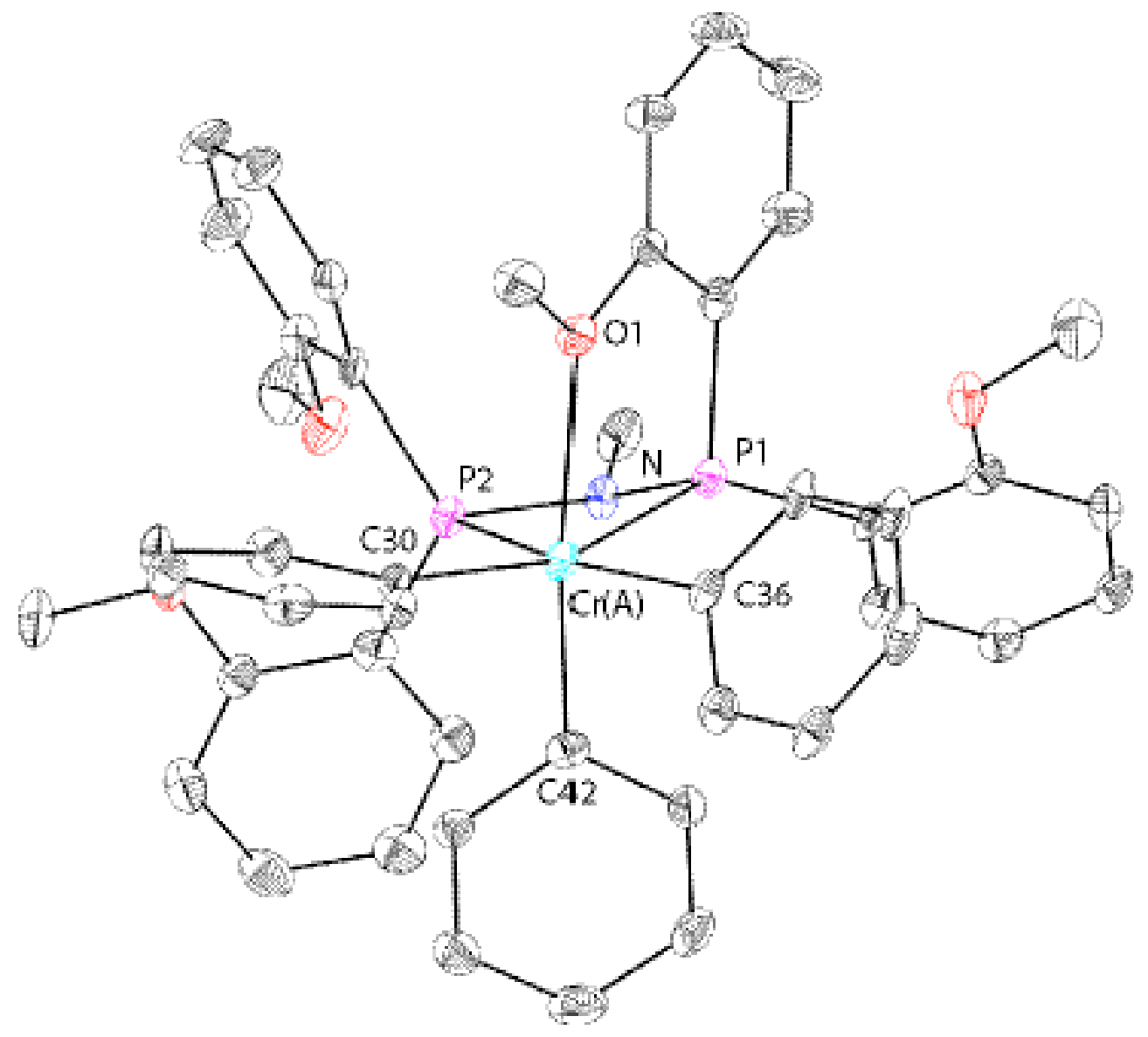

Figure 15. Structural drawing of $\mathbf{3}$ with thermal ellipsoids at the $50 \%$ probability level. 
Table 7. Crystal data and structure refinement for 3 (CCDC 216617).

Empirical formula

Formula weight

Crystallization Solvent

Crystal Habit

Crystal size

Crystal color

Preliminary Photos

Type of diffractometer

Wavelength

Data Collection Temperature

$\square$ range for 23120 reflections used

in lattice determination

Unit cell dimensions

Volume

Z

Crystal system

Space group

Density (calculated)

$\mathrm{F}(000)$

$\square$ range for data collection

Completeness to $\square=28.46^{\circ}$

Index ranges

Data collection scan type

Reflections collected

Independent reflections

Absorption coefficient

Absorption correction

Max. and min. transmission (predicted)
$\mathrm{C}_{47} \mathrm{H}_{46} \mathrm{NO}_{4} \mathrm{P}_{2} \mathrm{Cr} \cdot 3 \mathrm{CH}_{2} \mathrm{Cl}_{2}$

1057.57

Dichloromethane/petroleum ether

Block

$0.26 \times 0.17 \times 0.11 \mathrm{~mm}^{3}$

Dark red

\section{Data Collection}

Rotation

Bruker SMART 1000

$0.71073 \AA$ MoKロ

98(2) K

2.20 to $27.69^{\circ}$

$a=15.1624(7) \AA$

$\mathrm{b}=15.4024(7) \AA$

$\square=91.2320(10)^{\circ}$

$\square=94.4350(10)^{\circ}$

$\mathrm{c}=22.7168(11) \AA$

$\square=110.5250(10)^{\circ}$

4946.8(4) $\AA^{3}$

4

Triclinic

P-1

$1.420 \mathrm{Mg} / \mathrm{m}^{3}$

2188

1.41 to $28.46^{\circ}$

$91.7 \%$

$-20 \leq \mathrm{h} \leq 20,-20 \leq \mathrm{k} \leq 20,-30 \leq 1 \leq 29$

$\square$ scans at $7 \square$ settings

102892

$22906\left[\mathrm{R}_{\mathrm{int}}=0.0806\right]$

$0.665 \mathrm{~mm}^{-1}$

None

0.9304 and 0.8461 
Table 7 (cont.)

\section{Structure solution and Refinement}

Structure solution program

Primary solution method

Secondary solution method

Hydrogen placement

Structure refinement program

Refinement method

Data / restraints / parameters

Treatment of hydrogen atoms

Goodness-of-fit on $\mathrm{F}^{2}$

Final R indices [I $>2 \square(\mathrm{I}), 14122$ reflections]

$\mathrm{R}$ indices (all data)

Type of weighting scheme used

Weighting scheme used

Max shift/error

Average shift/error

Largest diff. peak and hole
SHELXS-97 (Sheldrick, 1990)

Direct methods

Difference Fourier map

Geometric positions

SHELXL-97 (Sheldrick, 1997)

Full matrix least-squares on $\mathrm{F}^{2}$

22906 / 0 / 1138

Constrained

2.003

$\mathrm{R} 1=0.0692, w \mathrm{R} 2=0.1163$

$\mathrm{R} 1=0.1196, w \mathrm{R} 2=0.1216$

Sigma

$w=1 / \square^{2}\left(\mathrm{Fo}^{2}\right)$

0.004

0.000

2.556 and -1.427 e. $\AA^{-3}$

\section{Special Refinement Details}

The crystals contain dichloromethane as solvent of crystallization. Each asymmetric unit contains six molecules of dichloromethane, four of which are occupied by distinct and ordered molecules ( $\mathrm{Cl} 1-8$ and $\mathrm{C} \mathrm{1-4).}$ These four were refined with anisotropic displacement parameters. The other two solvent sites are disordered and adjacent to each other. They are modeled by five molecules with partial occupancies ( $\mathrm{Cl} \mathrm{9-18} \mathrm{and} \mathrm{C} \mathrm{5-9).} \mathrm{Three} \mathrm{of}$ these five molecules ( $\mathrm{Cl}$ 13-18 and $\mathrm{C} \mathrm{7-9)}$ were included in the structure factor calculations but neither the coordinates nor temperature factors were refined during least-squares. Hydrogen atoms were restrained to ride on the atoms they are bonded to. No other restraints were applied during refinement.

Refinement of $\mathrm{F}^{2}$ against ALL reflections. The weighted R-factor $(w \mathrm{R})$ and goodness of fit $(\mathrm{S})$ are based on $\mathrm{F}^{2}$, conventional $\mathrm{R}$-factors $(\mathrm{R})$ are based on $\mathrm{F}$, with $\mathrm{F}$ set to zero for negative $\mathrm{F}^{2}$. The threshold expression of $\mathrm{F}^{2}>$ $2 \square\left(\mathrm{F}^{2}\right)$ is used only for calculating $\mathrm{R}$-factors $(\mathrm{gt})$ etc. and is not relevant to the choice of reflections for refinement. $\mathrm{R}$-factors based on $\mathrm{F}^{2}$ are statistically about twice as large as those based on $\mathrm{F}$, and R-factors based on ALL data will be even larger.

All esds (except the esd in the dihedral angle between two 1.s. planes) are estimated using the full covariance matrix. The cell esds are taken into account individually in the estimation of esds in distances, angles and torsion angles; correlations between esds in cell parameters are only used when they are defined by crystal symmetry. An approximate (isotropic) treatment of cell esds is used for estimating esds involving 1.s. planes. 
Table 8. Atomic coordinates $\left(\mathrm{x} \mathrm{10}^{4}\right)$ and equivalent isotropic displacement parameters $\left(\AA^{2} \times 10^{3}\right)$ for $3(C C D C 216617)$. $U(e q)$ is defined as the trace of the orthogonalized $U^{\mathrm{ij}}$ tensor.

\begin{tabular}{|c|c|c|c|c|c|}
\hline & $\mathrm{x}$ & $\mathrm{y}$ & $\mathrm{z}$ & $\mathrm{U}_{\mathrm{eq}}$ & Occ \\
\hline $\operatorname{Cr}(1)$ & $7825(1)$ & $8572(1)$ & $709(1)$ & $14(1)$ & 1 \\
\hline $\mathrm{P}(1 \mathrm{~A})$ & $7077(1)$ & $9574(1)$ & $1188(1)$ & $14(1)$ & 1 \\
\hline $\mathrm{P}(2 \mathrm{~A})$ & $7502(1)$ & $8284(1)$ & $1835(1)$ & $15(1)$ & 1 \\
\hline $\mathrm{O}(1 \mathrm{~A})$ & $9029(2)$ & $9938(2)$ & $1000(1)$ & $15(1)$ & 1 \\
\hline $\mathrm{O}(2 \mathrm{~A})$ & $7017(2)$ & $11087(2)$ & 453(1) & $21(1)$ & 1 \\
\hline $\mathrm{O}(3 \mathrm{~A})$ & $8011(2)$ & $6763(2)$ & $2332(1)$ & $22(1)$ & 1 \\
\hline $\mathrm{O}(4 \mathrm{~A})$ & $7334(2)$ & $8210(2)$ & $3176(1)$ & $23(1)$ & 1 \\
\hline $\mathrm{N}(1 \mathrm{~A})$ & $6824(2)$ & $8973(2)$ & $1810(1)$ & $14(1)$ & 1 \\
\hline $\mathrm{C}(1 \mathrm{~A})$ & $9953(2)$ & $10064(3)$ & 799(2) & $25(1)$ & 1 \\
\hline $\mathrm{C}(2 \mathrm{~A})$ & $8948(3)$ & $10702(3)$ & $1298(2)$ & $16(1)$ & 1 \\
\hline$C(3 A)$ & $8043(3)$ & $10635(3)$ & $1421(2)$ & $16(1)$ & 1 \\
\hline $\mathrm{C}(4 \mathrm{~A})$ & $7940(3)$ & $11384(3)$ & $1724(2)$ & $26(1)$ & 1 \\
\hline$C(5 \mathrm{~A})$ & $8698(3)$ & $12175(3)$ & $1895(2)$ & $35(1)$ & 1 \\
\hline$C(6 A)$ & $9595(3)$ & $12221(3)$ & $1765(2)$ & $29(1)$ & 1 \\
\hline $\mathrm{C}(7 \mathrm{~A})$ & $9724(3)$ & 11491(3) & $1464(2)$ & $20(1)$ & 1 \\
\hline $\mathrm{C}(8 \mathrm{~A})$ & $6069(2)$ & $9925(3)$ & $1008(2)$ & $13(1)$ & 1 \\
\hline $\mathrm{C}(9 \mathrm{~A})$ & $6157(3)$ & $10681(3)$ & $657(2)$ & $16(1)$ & 1 \\
\hline$C(10 A)$ & $7203(3)$ & $11946(3)$ & $185(2)$ & $32(1)$ & 1 \\
\hline $\mathrm{C}(11 \mathrm{~A})$ & $5392(3)$ & $10953(3)$ & $522(2)$ & $18(1)$ & 1 \\
\hline $\mathrm{C}(12 \mathrm{~A})$ & $4524(3)$ & $10466(3)$ & $717(2)$ & $19(1)$ & 1 \\
\hline$C(13 A)$ & $4421(3)$ & 9701(3) & $1047(2)$ & $19(1)$ & 1 \\
\hline $\mathrm{C}(14 \mathrm{~A})$ & $5182(2)$ & $9429(3)$ & 1191(2) & $17(1)$ & 1 \\
\hline $\mathrm{C}(15 \mathrm{~A})$ & $6705(3)$ & $7179(3)$ & $2048(2)$ & $18(1)$ & 1 \\
\hline$C(16 A)$ & $7046(3)$ & $6487(3)$ & $2251(2)$ & $18(1)$ & 1 \\
\hline $\mathrm{C}(17 \mathrm{~A})$ & $8396(3)$ & $6051(3)$ & $2431(2)$ & $27(1)$ & 1 \\
\hline $\mathrm{C}(18 \mathrm{~A})$ & $6439(3)$ & $5615(3)$ & $2345(2)$ & $24(1)$ & 1 \\
\hline$C(19 A)$ & $5473(3)$ & $5403(3)$ & $2239(2)$ & $28(1)$ & 1 \\
\hline$C(20 A)$ & $5124(3)$ & $6068(3)$ & $2035(2)$ & $25(1)$ & 1 \\
\hline $\mathrm{C}(21 \mathrm{~A})$ & $5742(3)$ & $6947(3)$ & 1941(2) & $20(1)$ & 1 \\
\hline $\mathrm{C}(22 \mathrm{~A})$ & $8406(3)$ & $8741(3)$ & $2455(2)$ & $16(1)$ & 1 \\
\hline $\mathrm{C}(23 \mathrm{~A})$ & $8245(3)$ & $8651(3)$ & $3054(2)$ & $22(1)$ & 1 \\
\hline $\mathrm{C}(24 \mathrm{~A})$ & $7127(3)$ & $8211(3)$ & $3779(2)$ & $34(1)$ & 1 \\
\hline$C(25 A)$ & 8999(3) & $9008(3)$ & $3484(2)$ & $31(1)$ & 1 \\
\hline$C(26 A)$ & 9892(3) & $9443(3)$ & $3326(2)$ & $35(1)$ & 1 \\
\hline $\mathrm{C}(27 \mathrm{~A})$ & $10063(3)$ & $9549(3)$ & 2741(2) & $26(1)$ & 1 \\
\hline $\mathrm{C}(28 \mathrm{~A})$ & $9326(3)$ & 9191(3) & $2310(2)$ & $19(1)$ & 1 \\
\hline$C(29 A)$ & $6567(3)$ & $9343(3)$ & $2347(2)$ & $21(1)$ & 1 \\
\hline$C(30 A)$ & $8741(2)$ & $7840(2)$ & $589(2)$ & $13(1)$ & 1 \\
\hline $\mathrm{C}(31 \mathrm{~A})$ & $9117(3)$ & $7749(3)$ & $59(2)$ & $18(1)$ & 1 \\
\hline $\mathrm{C}(32 \mathrm{~A})$ & 9813(3) & $7365(3)$ & $10(2)$ & $20(1)$ & 1 \\
\hline$C(33 A)$ & 10151(3) & $7029(3)$ & $504(2)$ & $22(1)$ & 1 \\
\hline $\mathrm{C}(34 \mathrm{~A})$ & $9782(3)$ & $7071(3)$ & $1038(2)$ & $22(1)$ & 1 \\
\hline$C(35 A)$ & $9101(3)$ & $7478(3)$ & $1077(2)$ & $21(1)$ & 1 \\
\hline$C(36 A)$ & $7763(2)$ & $9028(3)$ & $-141(2)$ & $14(1)$ & 1 \\
\hline$C(37 A)$ & $8088(2)$ & $9975(3)$ & $-269(2)$ & $18(1)$ & 1 \\
\hline $\mathrm{C}(38 \mathrm{~A})$ & $8042(3)$ & $10272(3)$ & $-835(2)$ & $21(1)$ & 1 \\
\hline $\mathrm{C}(39 \mathrm{~A})$ & $7667(3)$ & $9637(3)$ & $-1309(2)$ & $24(1)$ & 1 \\
\hline
\end{tabular}




\begin{tabular}{|c|c|c|c|c|c|}
\hline $\mathrm{C}(40 \mathrm{~A})$ & $7338(3)$ & $8697(3)$ & $-1210(2)$ & $22(1)$ & 1 \\
\hline $\mathrm{C}(41 \mathrm{~A})$ & $7383(3)$ & $8412(3)$ & $-636(2)$ & $20(1)$ & 1 \\
\hline $\mathrm{C}(42 \mathrm{~A})$ & $6585(3)$ & 7471(3) & $560(2)$ & $15(1)$ & 1 \\
\hline $\mathrm{C}(43 \mathrm{~A})$ & $6500(3)$ & 6555(3) & $682(2)$ & $16(1)$ & 1 \\
\hline $\mathrm{C}(44 \mathrm{~A})$ & $5654(3)$ & $5826(3)$ & $619(2)$ & $23(1)$ & 1 \\
\hline $\mathrm{C}(45 \mathrm{~A})$ & $4827(3)$ & 5979(3) & $421(2)$ & 28(1) & 1 \\
\hline C(46A) & $4876(3)$ & 6865(3) & $286(2)$ & $24(1)$ & 1 \\
\hline $\mathrm{C}(47 \mathrm{~A})$ & $5739(3)$ & 7591(3) & $355(2)$ & 19(1) & 1 \\
\hline $\mathrm{Cr}(2)$ & $1515(1)$ & 4732(1) & 3865(1) & $18(1)$ & 1 \\
\hline $\mathrm{P}(1 \mathrm{~B})$ & $3155(1)$ & 5916(1) & 3969(1) & $18(1)$ & 1 \\
\hline $\mathrm{P}(2 \mathrm{~B})$ & 2091(1) & $5590(1)$ & 2908(1) & 17(1) & 1 \\
\hline $\mathrm{O}(1 \mathrm{~B})$ & $1395(2)$ & 6061(2) & 4249(1) & 21(1) & 1 \\
\hline $\mathrm{O}(2 \mathrm{~B})$ & $3968(2)$ & $6235(2)$ & $5187(1)$ & $25(1)$ & 1 \\
\hline $\mathrm{O}(3 \mathrm{~B})$ & $748(2)$ & 4989(2) & 1850(1) & $25(1)$ & 1 \\
\hline $\mathrm{O}(4 \mathrm{~B})$ & $2743(2)$ & 6704(2) & $1829(1)$ & $25(1)$ & 1 \\
\hline $\mathrm{N}(1 \mathrm{~B})$ & $3224(2)$ & 6092(2) & $3235(1)$ & $16(1)$ & 1 \\
\hline$C(1 B)$ & $486(3)$ & 5970(3) & $4460(2)$ & $36(1)$ & 1 \\
\hline $\mathrm{C}(2 \mathrm{~B})$ & $2109(3)$ & 6911(3) & 4379(2) & 21(1) & 1 \\
\hline $\mathrm{C}(3 \mathrm{~B})$ & $3023(3)$ & 6956(3) & 4281(2) & 21(1) & 1 \\
\hline$C(4 B)$ & $3767(3)$ & 7797(3) & 4411(2) & $24(1)$ & 1 \\
\hline$C(5 B)$ & $3611(3)$ & $8575(3)$ & $4626(2)$ & $33(1)$ & 1 \\
\hline$C(6 B)$ & 2698(3) & $8509(3)$ & 4716(2) & $34(1)$ & 1 \\
\hline $\mathrm{C}(7 \mathrm{~B})$ & 1952(3) & 7689(3) & 4599(2) & 29(1) & 1 \\
\hline $\mathrm{C}(8 \mathrm{~B})$ & $4341(3)$ & 5986(3) & 4237(2) & 18(1) & 1 \\
\hline $\mathrm{C}(9 \mathrm{~B})$ & $4630(3)$ & 6147(3) & 4834(2) & $20(1)$ & 1 \\
\hline$C(10 B)$ & $4250(3)$ & $6525(3)$ & 5791(2) & $32(1)$ & 1 \\
\hline $\mathrm{C}(11 \mathrm{~B})$ & $5529(3)$ & 6188(3) & $5043(2)$ & $24(1)$ & 1 \\
\hline $\mathrm{C}(12 \mathrm{~B})$ & $6127(3)$ & 6043(3) & $4659(2)$ & $26(1)$ & 1 \\
\hline C(13B) & $5837(3)$ & $5849(3)$ & 4068(2) & $25(1)$ & 1 \\
\hline$C(14 B)$ & $4946(3)$ & 5821(3) & 3861(2) & $22(1)$ & 1 \\
\hline$C(15 B)$ & 2181(3) & 4921(3) & $2266(2)$ & $17(1)$ & 1 \\
\hline$C(16 B)$ & $1475(3)$ & 4673(3) & 1791(2) & 19(1) & 1 \\
\hline C(17B) & 4(3) & 4761(3) & 1389(2) & $27(1)$ & 1 \\
\hline $\mathrm{C}(18 \mathrm{~B})$ & $1538(3)$ & 4144(3) & 1304(2) & $23(1)$ & 1 \\
\hline$C(19 B)$ & $2284(3)$ & 3821(3) & 1297(2) & $26(1)$ & 1 \\
\hline$C(20 B)$ & 2958(3) & 4027(3) & 1767(2) & $22(1)$ & 1 \\
\hline$C(21 B)$ & 2910(3) & 4570(3) & $2246(2)$ & $18(1)$ & 1 \\
\hline$C(22 B)$ & $1800(3)$ & 6559(3) & $2623(2)$ & $18(1)$ & 1 \\
\hline$C(23 B)$ & 2161(3) & 7031(3) & 2121(2) & $22(1)$ & 1 \\
\hline$C(24 B)$ & 3034(3) & 7067(3) & $1282(2)$ & $36(1)$ & 1 \\
\hline$C(25 B)$ & 1925(3) & 7789(3) & 1942(2) & $30(1)$ & 1 \\
\hline$C(26 B)$ & 1333(3) & 8079(3) & $2266(2)$ & $34(1)$ & 1 \\
\hline$C(27 B)$ & 971(3) & $7626(3)$ & 2759(2) & $29(1)$ & 1 \\
\hline $\mathrm{C}(28 \mathrm{~B})$ & $1212(3)$ & 6881(3) & 2933(2) & 21(1) & 1 \\
\hline$C(29 B)$ & 3912(3) & 6945(3) & 3023(2) & $23(1)$ & 1 \\
\hline C(30B) & $65(3)$ & 4145(3) & $3609(2)$ & 19(1) & 1 \\
\hline C(31B) & $-574(3)$ & $3530(3)$ & $3946(2)$ & $30(1)$ & 1 \\
\hline $\mathrm{C}(32 \mathrm{~B})$ & $-1552(3)$ & $3186(3)$ & $3797(2)$ & $32(1)$ & 1 \\
\hline$C(33 B)$ & $-1913(3)$ & $3436(3)$ & $3282(2)$ & $27(1)$ & 1 \\
\hline$C(34 B)$ & $-1310(3)$ & 4027(3) & 2934(2) & $23(1)$ & 1 \\
\hline$C(35 B)$ & $-353(3)$ & 4384(3) & $3095(2)$ & 21(1) & 1 \\
\hline$C(36 B)$ & $1532(2)$ & $4220(3)$ & 4704(2) & $18(1)$ & 1 \\
\hline C(37B) & $1807(3)$ & $4785(3)$ & $5225(2)$ & $19(1)$ & 1 \\
\hline
\end{tabular}




\begin{tabular}{|c|c|c|c|c|c|}
\hline$C(38 B)$ & 1828(3) & $4446(3)$ & $5787(2)$ & $21(1)$ & 1 \\
\hline C(39B) & 1554(3) & 3502(3) & 5849(2) & $23(1)$ & 1 \\
\hline $\mathrm{C}(40 \mathrm{~B})$ & 1267(3) & 2900(3) & $5343(2)$ & $23(1)$ & 1 \\
\hline C(41B) & 1274(3) & $3264(3)$ & 4791(2) & $20(1)$ & 1 \\
\hline C(42B) & 1883(3) & 3694(3) & $3489(2)$ & $18(1)$ & 1 \\
\hline C(43B) & 2783(3) & 3639(3) & 3619(2) & 19(1) & 1 \\
\hline $\mathrm{C}(44 \mathrm{~B})$ & 3062(3) & 2975(3) & $3355(2)$ & $24(1)$ & 1 \\
\hline $\mathrm{C}(45 \mathrm{~B})$ & 2455(3) & 2315(3) & 2953(2) & $25(1)$ & 1 \\
\hline $\mathrm{C}(46 \mathrm{~B})$ & 1548(3) & 2332(3) & 2824(2) & $29(1)$ & 1 \\
\hline $\mathrm{C}(47 \mathrm{~B})$ & 1279(3) & $3007(3)$ & $3083(2)$ & $24(1)$ & 1 \\
\hline $\mathrm{Cl}(1)$ & 6394(1) & 2780(1) & 1849(1) & 61(1) & 1 \\
\hline $\mathrm{Cl}(2)$ & 4737(1) & 2689(1) & $2430(1)$ & 71(1) & 1 \\
\hline $\mathrm{C}(1)$ & 5199(4) & 2222(5) & 1883(3) & $130(4)$ & 1 \\
\hline $\mathrm{Cl}(3)$ & 8613(1) & 4531(1) & $22(1)$ & $36(1)$ & 1 \\
\hline $\mathrm{Cl}(4)$ & 7333(1) & $4550(1)$ & 902(1) & $37(1)$ & 1 \\
\hline $\mathrm{C}(2)$ & 7905(3) & $5117(3)$ & $298(2)$ & $28(1)$ & 1 \\
\hline $\mathrm{Cl}(5)$ & 4099(1) & 9532(1) & 2551(1) & $86(1)$ & 1 \\
\hline $\mathrm{Cl}(6)$ & 2836(1) & 10173(1) & $3146(1)$ & 91(1) & 1 \\
\hline $\mathrm{C}(3)$ & $3503(5)$ & $9438(5)$ & $3167(3)$ & $96(3)$ & 1 \\
\hline $\mathrm{Cl}(7)$ & $3565(1)$ & $1280(1)$ & 4694(1) & $79(1)$ & 1 \\
\hline $\mathrm{Cl}(8)$ & $1534(1)$ & $675(1)$ & $4738(1)$ & $56(1)$ & 1 \\
\hline $\mathrm{C}(4)$ & 2553(4) & 1538(3) & $4517(2)$ & $55(2)$ & 1 \\
\hline $\mathrm{Cl}(9)$ & $9001(1)$ & $1035(1)$ & $4340(1)$ & $35(1)$ & $0.602(3)$ \\
\hline $\mathrm{Cl}(10)$ & $8838(1)$ & $1157(1)$ & $3074(1)$ & $35(1)$ & $0.602(3)$ \\
\hline$C(5)$ & 9571(5) & 1432(5) & $3723(3)$ & $30(2)$ & $0.602(3)$ \\
\hline $\mathrm{Cl}(11)$ & $4262(2)$ & $8576(2)$ & 6614(2) & $50(1)$ & $0.389(4)$ \\
\hline $\mathrm{Cl}(12)$ & 4103(2) & 10233(2) & 6090(2) & $54(1)$ & $0.389(4)$ \\
\hline$C(6)$ & $3600(8)$ & 9167(8) & 6392(6) & 41(3) & $0.389(4)$ \\
\hline $\mathrm{Cl}(13)$ & 4336 & 8578 & 6283 & 70 & $0.197(4)$ \\
\hline $\mathrm{Cl}(14)$ & 4111 & 10223 & 6082 & 70 & $0.197(4)$ \\
\hline$C(7)$ & 3440 & 9101 & 6181 & 70 & $0.197(4)$ \\
\hline $\mathrm{Cl}(15)$ & 2504 & 9206 & 6381 & 70 & $0.120(2)$ \\
\hline $\mathrm{Cl}(16)$ & 4837 & 9389 & 6268 & 70 & $0.120(2)$ \\
\hline$C(8)$ & 3440 & 8857 & 6095 & 70 & $0.120(2)$ \\
\hline $\mathrm{Cl}(17)$ & 2510 & 9200 & 6380 & 70 & $0.230(3)$ \\
\hline $\mathrm{Cl}(18)$ & 9470 & 1217 & 4174 & 70 & $0.230(3)$ \\
\hline $\mathrm{C}(9)$ & 8690 & 1150 & 3519 & 70 & $0.230(3)$ \\
\hline
\end{tabular}


Table 9. Selected bond lengths $[\AA ̊ \AA]$ and angles $\left[{ }^{\circ}\right]$ for 3 (CCDC 216617).

$\begin{array}{lc}\mathrm{Cr}(1)-\mathrm{C}(42 \mathrm{~A}) & 2.041(4) \\ \mathrm{Cr}(1)-\mathrm{C}(36 \mathrm{~A}) & 2.075(4) \\ \mathrm{Cr}(1)-\mathrm{C}(30 \mathrm{~A}) & 2.101(4) \\ \mathrm{Cr}(1)-\mathrm{O}(1 \mathrm{~A}) & 2.293(2) \\ \mathrm{Cr}(1)-\mathrm{P}(1 \mathrm{~A}) & 2.4897(11) \\ \mathrm{Cr}(1)-\mathrm{P}(2 \mathrm{~A}) & 2.6597(12) \\ \mathrm{Cr}(2)-\mathrm{C}(42 \mathrm{~B}) & 2.060(4) \\ \mathrm{Cr}(2)-\mathrm{C}(36 \mathrm{~B}) & 2.081(4) \\ \mathrm{Cr}(2)-\mathrm{C}(30 \mathrm{~B}) & 2.092(4) \\ \mathrm{Cr}(2)-\mathrm{O}(1 \mathrm{~B}) & 2.279(3) \\ \mathrm{Cr}(2)-\mathrm{P}(1 \mathrm{~B}) & 2.5044(12) \\ \mathrm{Cr}(2)-\mathrm{P}(2 \mathrm{~B}) & 2.6164(12) \\ \mathrm{C}(42 \mathrm{~A})-\mathrm{Cr}(1)-\mathrm{C}(36 \mathrm{~A}) & 94.05(15) \\ \mathrm{C}(42 \mathrm{~A})-\mathrm{Cr}(1)-\mathrm{C}(30 \mathrm{~A}) & 97.35(14) \\ \mathrm{C}(36 \mathrm{~A})-\mathrm{Cr}(1)-\mathrm{C}(30 \mathrm{~A}) & 98.06(14) \\ \mathrm{C}(42 \mathrm{~A})-\mathrm{Cr}(1)-\mathrm{O}(1 \mathrm{~A}) & 167.42(12) \\ \mathrm{C}(36 \mathrm{~A})-\mathrm{Cr}(1)-\mathrm{O}(1 \mathrm{~A}) & 89.98(12) \\ \mathrm{C}(30 \mathrm{~A})-\mathrm{Cr}(1)-\mathrm{O}(1 \mathrm{~A}) & 93.88(11) \\ \mathrm{C}(42 \mathrm{~A})-\mathrm{Cr}(1)-\mathrm{P}(1 \mathrm{~A}) & 93.71(11) \\ \mathrm{C}(36 \mathrm{~A})-\mathrm{Cr}(1)-\mathrm{P}(1 \mathrm{~A}) & 97.71(10) \\ \mathrm{C}(30 \mathrm{~A})-\mathrm{Cr}(1)-\mathrm{P}(1 \mathrm{~A}) & 160.02(10) \\ \mathrm{O}(1 \mathrm{~A})-\mathrm{Cr}(1)-\mathrm{P}(1 \mathrm{~A}) & 73.92(7) \\ & \end{array}$

$\mathrm{C}(42 \mathrm{~A})-\mathrm{Cr}(1)-\mathrm{P}(2 \mathrm{~A})$

$\mathrm{C}(36 \mathrm{~A})-\mathrm{Cr}(1)-\mathrm{P}(2 \mathrm{~A})$

$\mathrm{C}(30 \mathrm{~A})-\mathrm{Cr}(1)-\mathrm{P}(2 \mathrm{~A})$

$\mathrm{O}(1 \mathrm{~A})-\mathrm{Cr}(1)-\mathrm{P}(2 \mathrm{~A})$

$\mathrm{P}(1 \mathrm{~A})-\mathrm{Cr}(1)-\mathrm{P}(2 \mathrm{~A})$

C(42B)-Cr(2)-C(36B)

$\mathrm{C}(42 \mathrm{~B})-\mathrm{Cr}(2)-\mathrm{C}(30 \mathrm{~B})$

$\mathrm{C}(36 \mathrm{~B})-\mathrm{Cr}(2)-\mathrm{C}(30 \mathrm{~B})$

$\mathrm{C}(42 \mathrm{~B})-\mathrm{Cr}(2)-\mathrm{O}(1 \mathrm{~B})$

$\mathrm{C}(36 \mathrm{~B})-\mathrm{Cr}(2)-\mathrm{O}(1 \mathrm{~B})$

$\mathrm{C}(30 \mathrm{~B})-\mathrm{Cr}(2)-\mathrm{O}(1 \mathrm{~B})$

$\mathrm{C}(42 \mathrm{~B})-\mathrm{Cr}(2)-\mathrm{P}(1 \mathrm{~B})$

$\mathrm{C}(36 \mathrm{~B})-\mathrm{Cr}(2)-\mathrm{P}(1 \mathrm{~B})$

$\mathrm{C}(30 \mathrm{~B})-\mathrm{Cr}(2)-\mathrm{P}(1 \mathrm{~B})$

$\mathrm{O}(1 \mathrm{~B})-\mathrm{Cr}(2)-\mathrm{P}(1 \mathrm{~B})$

$\mathrm{C}(42 \mathrm{~B})-\mathrm{Cr}(2)-\mathrm{P}(2 \mathrm{~B})$

$\mathrm{C}(36 \mathrm{~B})-\mathrm{Cr}(2)-\mathrm{P}(2 \mathrm{~B})$

$\mathrm{C}(30 \mathrm{~B})-\mathrm{Cr}(2)-\mathrm{P}(2 \mathrm{~B})$

$\mathrm{O}(1 \mathrm{~B})-\mathrm{Cr}(2)-\mathrm{P}(2 \mathrm{~B})$

$\mathrm{P}(1 \mathrm{~B})-\mathrm{Cr}(2)-\mathrm{P}(2 \mathrm{~B})$
$82.90(10)$

$160.72(10)$

$101.20(10)$

$89.37(7)$

63.67(3)

$92.86(15)$

96.62(15)

$99.25(15)$

168.94(13)

$90.87(13)$

93.04(12)

95.52(11)

97.63(11)

158.64(12)

73.66(7)

83.95(11)

161.18(11)

99.55(11)

89.16(7)

64.37(4) 
Table 10. Bond lengths $[\AA ̊ \AA]$ and angles $\left[^{\circ}\right]$ for 3 (CCDC 216617).

\begin{tabular}{|c|c|c|c|}
\hline & & $\mathrm{C}(32 \mathrm{~A})-\mathrm{C}(33 \mathrm{~A})$ & $1.382(5)$ \\
\hline & & $C(33 A)-C(34 A)$ & $1.385(5)$ \\
\hline $\mathrm{Cr}(1)-\mathrm{C}(42 \mathrm{~A})$ & $2.041(4)$ & $\mathrm{C}(34 \mathrm{~A})-\mathrm{C}(35 \mathrm{~A})$ & $1.390(5)$ \\
\hline $\mathrm{Cr}(1)-\mathrm{C}(36 \mathrm{~A})$ & $2.075(4)$ & $C(36 \mathrm{~A})-\mathrm{C}(41 \mathrm{~A})$ & $1.401(5)$ \\
\hline $\mathrm{Cr}(1)-\mathrm{C}(30 \mathrm{~A})$ & $2.101(4)$ & $C(36 \mathrm{~A})-\mathrm{C}(37 \mathrm{~A})$ & $1.412(5)$ \\
\hline $\mathrm{Cr}(1)-\mathrm{O}(1 \mathrm{~A})$ & $2.293(2)$ & $\mathrm{C}(37 \mathrm{~A})-\mathrm{C}(38 \mathrm{~A})$ & $1.380(5)$ \\
\hline $\mathrm{Cr}(1)-\mathrm{P}(1 \mathrm{~A})$ & $2.4897(11)$ & $\mathrm{C}(38 \mathrm{~A})-\mathrm{C}(39 \mathrm{~A})$ & $1.381(5)$ \\
\hline $\mathrm{Cr}(1)-\mathrm{P}(2 \mathrm{~A})$ & $2.6597(12)$ & $C(39 A)-C(40 A)$ & $1.387(5)$ \\
\hline $\mathrm{P}(1 \mathrm{~A})-\mathrm{N}(1 \mathrm{~A})$ & $1.700(3)$ & $\mathrm{C}(40 \mathrm{~A})-\mathrm{C}(41 \mathrm{~A})$ & $1.390(5)$ \\
\hline$P(1 \mathrm{~A})-\mathrm{C}(3 \mathrm{~A})$ & $1.806(4)$ & $\mathrm{C}(42 \mathrm{~A})-\mathrm{C}(47 \mathrm{~A})$ & $1.405(5)$ \\
\hline $\mathrm{P}(1 \mathrm{~A})-\mathrm{C}(8 \mathrm{~A})$ & $1.814(4)$ & $\mathrm{C}(42 \mathrm{~A})-\mathrm{C}(43 \mathrm{~A})$ & $1.407(5)$ \\
\hline $\mathrm{P}(2 \mathrm{~A})-\mathrm{N}(1 \mathrm{~A})$ & $1.716(3)$ & $\mathrm{C}(43 \mathrm{~A})-\mathrm{C}(44 \mathrm{~A})$ & $1.372(5)$ \\
\hline $\mathrm{P}(2 \mathrm{~A})-\mathrm{C}(15 \mathrm{~A})$ & $1.810(4)$ & $\mathrm{C}(44 \mathrm{~A})-\mathrm{C}(45 \mathrm{~A})$ & $1.398(5)$ \\
\hline $\mathrm{P}(2 \mathrm{~A})-\mathrm{C}(22 \mathrm{~A})$ & $1.827(4)$ & $\mathrm{C}(45 \mathrm{~A})-\mathrm{C}(46 \mathrm{~A})$ & $1.382(5)$ \\
\hline $\mathrm{O}(1 \mathrm{~A})-\mathrm{C}(2 \mathrm{~A})$ & $1.391(4)$ & $\mathrm{C}(46 \mathrm{~A})-\mathrm{C}(47 \mathrm{~A})$ & $1.386(5)$ \\
\hline $\mathrm{O}(1 \mathrm{~A})-\mathrm{C}(1 \mathrm{~A})$ & $1.457(4)$ & $\mathrm{Cr}(2)-\mathrm{C}(42 \mathrm{~B})$ & $2.060(4)$ \\
\hline $\mathrm{O}(2 \mathrm{~A})-\mathrm{C}(9 \mathrm{~A})$ & $1.356(4)$ & $\mathrm{Cr}(2)-\mathrm{C}(36 \mathrm{~B})$ & $2.081(4)$ \\
\hline $\mathrm{O}(2 \mathrm{~A})-\mathrm{C}(10 \mathrm{~A})$ & $1.414(4)$ & $\mathrm{Cr}(2)-\mathrm{C}(30 \mathrm{~B})$ & $2.092(4)$ \\
\hline $\mathrm{O}(3 \mathrm{~A})-\mathrm{C}(16 \mathrm{~A})$ & $1.369(4)$ & $\mathrm{Cr}(2)-\mathrm{O}(1 \mathrm{~B})$ & $2.279(3)$ \\
\hline $\mathrm{O}(3 \mathrm{~A})-\mathrm{C}(17 \mathrm{~A})$ & $1.426(4)$ & $\mathrm{Cr}(2)-\mathrm{P}(1 \mathrm{~B})$ & $2.5044(12)$ \\
\hline $\mathrm{O}(4 \mathrm{~A})-\mathrm{C}(23 \mathrm{~A})$ & $1.362(4)$ & $\mathrm{Cr}(2)-\mathrm{P}(2 \mathrm{~B})$ & $2.6164(12)$ \\
\hline $\mathrm{O}(4 \mathrm{~A})-\mathrm{C}(24 \mathrm{~A})$ & $1.429(4)$ & $\mathrm{P}(1 \mathrm{~B})-\mathrm{N}(1 \mathrm{~B})$ & $1.702(3)$ \\
\hline $\mathrm{N}(1 \mathrm{~A})-\mathrm{C}(29 \mathrm{~A})$ & $1.472(4)$ & $\mathrm{P}(1 \mathrm{~B})-\mathrm{C}(8 \mathrm{~B})$ & $1.819(4)$ \\
\hline $\mathrm{C}(2 \mathrm{~A})-\mathrm{C}(7 \mathrm{~A})$ & $1.383(5)$ & $\mathrm{P}(1 \mathrm{~B})-\mathrm{C}(3 \mathrm{~B})$ & $1.819(4)$ \\
\hline $\mathrm{C}(2 \mathrm{~A})-\mathrm{C}(3 \mathrm{~A})$ & $1.391(5)$ & $\mathrm{P}(2 \mathrm{~B})-\mathrm{N}(1 \mathrm{~B})$ & $1.718(3)$ \\
\hline $\mathrm{C}(3 \mathrm{~A})-\mathrm{C}(4 \mathrm{~A})$ & $1.392(5)$ & $\mathrm{P}(2 \mathrm{~B})-\mathrm{C}(15 \mathrm{~B})$ & $1.807(4)$ \\
\hline$C(4 A)-C(5 A)$ & $1.372(5)$ & $\mathrm{P}(2 \mathrm{~B})-\mathrm{C}(22 \mathrm{~B})$ & $1.816(4)$ \\
\hline$C(5 A)-C(6 A)$ & $1.393(5)$ & $\mathrm{O}(1 \mathrm{~B})-\mathrm{C}(2 \mathrm{~B})$ & $1.383(4)$ \\
\hline$C(6 A)-C(7 A)$ & $1.383(5)$ & $\mathrm{O}(1 \mathrm{~B})-\mathrm{C}(1 \mathrm{~B})$ & $1.454(4)$ \\
\hline $\mathrm{C}(8 \mathrm{~A})-\mathrm{C}(9 \mathrm{~A})$ & $1.398(5)$ & $\mathrm{O}(2 \mathrm{~B})-\mathrm{C}(9 \mathrm{~B})$ & $1.372(4)$ \\
\hline$C(8 \mathrm{~A})-\mathrm{C}(14 \mathrm{~A})$ & $1.395(5)$ & $\mathrm{O}(2 \mathrm{~B})-\mathrm{C}(10 \mathrm{~B})$ & $1.419(4)$ \\
\hline $\mathrm{C}(9 \mathrm{~A})-\mathrm{C}(11 \mathrm{~A})$ & $1.381(5)$ & $\mathrm{O}(3 \mathrm{~B})-\mathrm{C}(16 \mathrm{~B})$ & $1.367(4)$ \\
\hline$C(11 A)-C(12 A)$ & $1.380(5)$ & $\mathrm{O}(3 \mathrm{~B})-\mathrm{C}(17 \mathrm{~B})$ & $1.421(4)$ \\
\hline$C(12 A)-C(13 A)$ & $1.380(5)$ & $\mathrm{O}(4 \mathrm{~B})-\mathrm{C}(23 \mathrm{~B})$ & $1.365(4)$ \\
\hline$C(13 A)-C(14 A)$ & $1.378(5)$ & $\mathrm{O}(4 \mathrm{~B})-\mathrm{C}(24 \mathrm{~B})$ & $1.412(4)$ \\
\hline$C(15 \mathrm{~A})-\mathrm{C}(21 \mathrm{~A})$ & $1.376(5)$ & $\mathrm{N}(1 \mathrm{~B})-\mathrm{C}(29 \mathrm{~B})$ & $1.479(5)$ \\
\hline$C(15 A)-C(16 A)$ & $1.409(5)$ & $\mathrm{C}(2 \mathrm{~B})-\mathrm{C}(7 \mathrm{~B})$ & $1.391(5)$ \\
\hline$C(16 A)-C(18 A)$ & $1.369(5)$ & $\mathrm{C}(2 \mathrm{~B})-\mathrm{C}(3 \mathrm{~B})$ & $1.398(5)$ \\
\hline$C(18 A)-C(19 A)$ & $1.384(5)$ & $\mathrm{C}(3 \mathrm{~B})-\mathrm{C}(4 \mathrm{~B})$ & $1.395(5)$ \\
\hline$C(19 A)-C(20 A)$ & $1.380(5)$ & $\mathrm{C}(4 \mathrm{~B})-\mathrm{C}(5 \mathrm{~B})$ & $1.386(5)$ \\
\hline$C(20 A)-C(21 A)$ & $1.382(5)$ & $\mathrm{C}(5 \mathrm{~B})-\mathrm{C}(6 \mathrm{~B})$ & $1.384(6)$ \\
\hline$C(22 A)-C(28 A)$ & $1.393(5)$ & $\mathrm{C}(6 \mathrm{~B})-\mathrm{C}(7 \mathrm{~B})$ & $1.372(6)$ \\
\hline$C(22 A)-C(23 A)$ & $1.402(5)$ & $\mathrm{C}(8 \mathrm{~B})-\mathrm{C}(9 \mathrm{~B})$ & $1.382(5)$ \\
\hline$C(23 \mathrm{~A})-\mathrm{C}(25 \mathrm{~A})$ & $1.388(5)$ & $\mathrm{C}(8 \mathrm{~B})-\mathrm{C}(14 \mathrm{~B})$ & $1.383(5)$ \\
\hline$C(25 A)-C(26 A)$ & $1.364(6)$ & $\mathrm{C}(9 \mathrm{~B})-\mathrm{C}(11 \mathrm{~B})$ & $1.387(5)$ \\
\hline$C(26 A)-C(27 A)$ & $1.377(6)$ & $\mathrm{C}(11 \mathrm{~B})-\mathrm{C}(12 \mathrm{~B})$ & $1.373(5)$ \\
\hline$C(27 A)-C(28 A)$ & $1.373(5)$ & $\mathrm{C}(12 \mathrm{~B})-\mathrm{C}(13 \mathrm{~B})$ & $1.374(5)$ \\
\hline$C(30 A)-C(31 A)$ & $1.396(5)$ & $\mathrm{C}(13 \mathrm{~B})-\mathrm{C}(14 \mathrm{~B})$ & $1.381(5)$ \\
\hline$C(30 A)-C(35 A)$ & $1.410(5)$ & $\mathrm{C}(15 \mathrm{~B})-\mathrm{C}(21 \mathrm{~B})$ & $1.393(5)$ \\
\hline $\mathrm{C}(31 \mathrm{~A})-\mathrm{C}(32 \mathrm{~A})$ & $1.389(5)$ & $\mathrm{C}(15 \mathrm{~B})-\mathrm{C}(16 \mathrm{~B})$ & $1.406(5)$ \\
\hline
\end{tabular}




\begin{tabular}{|c|c|c|c|}
\hline$C(16 B)-C(18 B)$ & $1.389(5)$ & $\mathrm{C}(42 \mathrm{~A})-\mathrm{Cr}(1)-\mathrm{P}(1 \mathrm{~A})$ & $93.71(11)$ \\
\hline$C(18 B)-C(19 B)$ & $1.387(5)$ & $\mathrm{C}(36 \mathrm{~A})-\mathrm{Cr}(1)-\mathrm{P}(1 \mathrm{~A})$ & $97.71(10)$ \\
\hline$C(19 B)-C(20 B)$ & $1.368(5)$ & $\mathrm{C}(30 \mathrm{~A})-\mathrm{Cr}(1)-\mathrm{P}(1 \mathrm{~A})$ & $160.02(10)$ \\
\hline$C(20 B)-C(21 B)$ & $1.381(5)$ & $\mathrm{O}(1 \mathrm{~A})-\mathrm{Cr}(1)-\mathrm{P}(1 \mathrm{~A})$ & $73.92(7)$ \\
\hline $\mathrm{C}(22 \mathrm{~B})-\mathrm{C}(23 \mathrm{~B})$ & $1.408(5)$ & $\mathrm{C}(42 \mathrm{~A})-\mathrm{Cr}(1)-\mathrm{P}(2 \mathrm{~A})$ & $82.90(10)$ \\
\hline $\mathrm{C}(22 \mathrm{~B})-\mathrm{C}(28 \mathrm{~B})$ & $1.388(5)$ & $\mathrm{C}(36 \mathrm{~A})-\mathrm{Cr}(1)-\mathrm{P}(2 \mathrm{~A})$ & $160.72(10)$ \\
\hline$C(23 B)-C(25 B)$ & $1.395(5)$ & $\mathrm{C}(30 \mathrm{~A})-\mathrm{Cr}(1)-\mathrm{P}(2 \mathrm{~A})$ & $101.20(10)$ \\
\hline$C(25 B)-C(26 B)$ & $1.384(6)$ & $\mathrm{O}(1 \mathrm{~A})-\mathrm{Cr}(1)-\mathrm{P}(2 \mathrm{~A})$ & $89.37(7)$ \\
\hline $\mathrm{C}(26 \mathrm{~B})-\mathrm{C}(27 \mathrm{~B})$ & $1.379(6)$ & $\mathrm{P}(1 \mathrm{~A})-\mathrm{Cr}(1)-\mathrm{P}(2 \mathrm{~A})$ & $63.67(3)$ \\
\hline $\mathrm{C}(27 \mathrm{~B})-\mathrm{C}(28 \mathrm{~B})$ & $1.377(5)$ & $\mathrm{N}(1 \mathrm{~A})-\mathrm{P}(1 \mathrm{~A})-\mathrm{C}(3 \mathrm{~A})$ & $105.38(16)$ \\
\hline$C(30 B)-C(31 B)$ & $1.388(5)$ & $\mathrm{N}(1 \mathrm{~A})-\mathrm{P}(1 \mathrm{~A})-\mathrm{C}(8 \mathrm{~A})$ & $104.71(16)$ \\
\hline$C(30 B)-C(35 B)$ & $1.404(5)$ & $\mathrm{C}(3 \mathrm{~A})-\mathrm{P}(1 \mathrm{~A})-\mathrm{C}(8 \mathrm{~A})$ & $105.16(17)$ \\
\hline $\mathrm{C}(31 \mathrm{~B})-\mathrm{C}(32 \mathrm{~B})$ & $1.399(5)$ & $\mathrm{N}(1 \mathrm{~A})-\mathrm{P}(1 \mathrm{~A})-\mathrm{Cr}(1)$ & $97.02(11)$ \\
\hline $\mathrm{C}(32 \mathrm{~B})-\mathrm{C}(33 \mathrm{~B})$ & $1.372(5)$ & $\mathrm{C}(3 \mathrm{~A})-\mathrm{P}(1 \mathrm{~A})-\mathrm{Cr}(1)$ & $104.99(12)$ \\
\hline $\mathrm{C}(33 \mathrm{~B})-\mathrm{C}(34 \mathrm{~B})$ & $1.359(5)$ & $\mathrm{C}(8 \mathrm{~A})-\mathrm{P}(1 \mathrm{~A})-\mathrm{Cr}(1)$ & $136.20(12)$ \\
\hline $\mathrm{C}(34 \mathrm{~B})-\mathrm{C}(35 \mathrm{~B})$ & $1.375(5)$ & $\mathrm{N}(1 \mathrm{~A})-\mathrm{P}(2 \mathrm{~A})-\mathrm{C}(15 \mathrm{~A})$ & $103.74(17)$ \\
\hline $\mathrm{C}(36 \mathrm{~B})-\mathrm{C}(37 \mathrm{~B})$ & $1.399(5)$ & $\mathrm{N}(1 \mathrm{~A})-\mathrm{P}(2 \mathrm{~A})-\mathrm{C}(22 \mathrm{~A})$ & $106.92(16)$ \\
\hline$C(36 B)-C(41 B)$ & $1.408(5)$ & $\mathrm{C}(15 \mathrm{~A})-\mathrm{P}(2 \mathrm{~A})-\mathrm{C}(22 \mathrm{~A})$ & $106.40(18)$ \\
\hline $\mathrm{C}(37 \mathrm{~B})-\mathrm{C}(38 \mathrm{~B})$ & $1.392(5)$ & $\mathrm{N}(1 \mathrm{~A})-\mathrm{P}(2 \mathrm{~A})-\mathrm{Cr}(1)$ & $90.67(11)$ \\
\hline $\mathrm{C}(38 \mathrm{~B})-\mathrm{C}(39 \mathrm{~B})$ & $1.379(5)$ & $\mathrm{C}(15 \mathrm{~A})-\mathrm{P}(2 \mathrm{~A})-\mathrm{Cr}(1)$ & $120.71(13)$ \\
\hline$C(39 B)-C(40 B)$ & $1.400(5)$ & $\mathrm{C}(22 \mathrm{~A})-\mathrm{P}(2 \mathrm{~A})-\mathrm{Cr}(1)$ & $123.86(13)$ \\
\hline$C(40 B)-C(41 B)$ & $1.383(5)$ & $C(2 A)-O(1 A)-C(1 A)$ & $116.0(3)$ \\
\hline $\mathrm{C}(42 \mathrm{~B})-\mathrm{C}(47 \mathrm{~B})$ & $1.400(5)$ & $\mathrm{C}(2 \mathrm{~A})-\mathrm{O}(1 \mathrm{~A})-\mathrm{Cr}(1)$ & $126.6(2)$ \\
\hline$C(42 B)-C(43 B)$ & $1.404(5)$ & $\mathrm{C}(1 \mathrm{~A})-\mathrm{O}(1 \mathrm{~A})-\mathrm{Cr}(1)$ & $117.0(2)$ \\
\hline$C(43 B)-C(44 B)$ & $1.378(5)$ & $\mathrm{C}(9 \mathrm{~A})-\mathrm{O}(2 \mathrm{~A})-\mathrm{C}(10 \mathrm{~A})$ & $118.2(3)$ \\
\hline $\mathrm{C}(44 \mathrm{~B})-\mathrm{C}(45 \mathrm{~B})$ & $1.374(5)$ & $\mathrm{C}(16 \mathrm{~A})-\mathrm{O}(3 \mathrm{~A})-\mathrm{C}(17 \mathrm{~A})$ & $116.6(3)$ \\
\hline$C(45 B)-C(46 B)$ & $1.392(5)$ & $\mathrm{C}(23 \mathrm{~A})-\mathrm{O}(4 \mathrm{~A})-\mathrm{C}(24 \mathrm{~A})$ & $117.9(3)$ \\
\hline$C(46 B)-C(47 B)$ & $1.378(5)$ & $\mathrm{C}(29 \mathrm{~A})-\mathrm{N}(1 \mathrm{~A})-\mathrm{P}(1 \mathrm{~A})$ & $123.6(3)$ \\
\hline $\mathrm{Cl}(1)-\mathrm{C}(1)$ & $1.721(6)$ & $C(29 A)-N(1 A)-P(2 A)$ & $122.4(2)$ \\
\hline $\mathrm{Cl}(2)-\mathrm{C}(1)$ & $1.733(5)$ & $\mathrm{P}(1 \mathrm{~A})-\mathrm{N}(1 \mathrm{~A})-\mathrm{P}(2 \mathrm{~A})$ & $105.52(16)$ \\
\hline $\mathrm{Cl}(3)-\mathrm{C}(2)$ & $1.767(4)$ & $\mathrm{C}(7 \mathrm{~A})-\mathrm{C}(2 \mathrm{~A})-\mathrm{C}(3 \mathrm{~A})$ & $121.3(4)$ \\
\hline $\mathrm{Cl}(4)-\mathrm{C}(2)$ & $1.762(4)$ & $\mathrm{C}(7 \mathrm{~A})-\mathrm{C}(2 \mathrm{~A})-\mathrm{O}(1 \mathrm{~A})$ & $122.2(3)$ \\
\hline $\mathrm{Cl}(5)-\mathrm{C}(3)$ & $1.706(6)$ & $\mathrm{C}(3 \mathrm{~A})-\mathrm{C}(2 \mathrm{~A})-\mathrm{O}(1 \mathrm{~A})$ & $116.6(3)$ \\
\hline $\mathrm{Cl}(6)-\mathrm{C}(3)$ & $1.761(5)$ & $C(4 A)-C(3 A)-C(2 A)$ & $118.0(4)$ \\
\hline $\mathrm{Cl}(7)-\mathrm{C}(4)$ & $1.737(5)$ & $\mathrm{C}(4 \mathrm{~A})-\mathrm{C}(3 \mathrm{~A})-\mathrm{P}(1 \mathrm{~A})$ & 124.1(3) \\
\hline $\mathrm{Cl}(8)-\mathrm{C}(4)$ & $1.764(5)$ & $\mathrm{C}(2 \mathrm{~A})-\mathrm{C}(3 \mathrm{~A})-\mathrm{P}(1 \mathrm{~A})$ & $117.9(3)$ \\
\hline $\mathrm{Cl}(9)-\mathrm{C}(5)$ & $1.715(7)$ & $C(3 A)-C(4 A)-C(5 A)$ & $122.0(4)$ \\
\hline $\mathrm{Cl}(10)-\mathrm{C}(5)$ & $1.726(7)$ & $C(6 A)-C(5 A)-C(4 A)$ & $118.7(4)$ \\
\hline $\mathrm{Cl}(11)-\mathrm{C}(6)$ & $1.634(12)$ & $C(7 A)-C(6 A)-C(5 A)$ & $120.9(4)$ \\
\hline $\mathrm{Cl}(12)-\mathrm{C}(6)$ & $1.731(12)$ & $\mathrm{C}(6 \mathrm{~A})-\mathrm{C}(7 \mathrm{~A})-\mathrm{C}(2 \mathrm{~A})$ & 119.1(4) \\
\hline $\mathrm{Cl}(13)-\mathrm{C}(7)$ & 1.8092 & $\mathrm{C}(9 \mathrm{~A})-\mathrm{C}(8 \mathrm{~A})-\mathrm{C}(14 \mathrm{~A})$ & 118.4(3) \\
\hline $\mathrm{Cl}(14)-\mathrm{C}(7)$ & 1.7016 & $\mathrm{C}(9 \mathrm{~A})-\mathrm{C}(8 \mathrm{~A})-\mathrm{P}(1 \mathrm{~A})$ & $120.3(3)$ \\
\hline $\mathrm{Cl}(15)-\mathrm{C}(8)$ & 1.8406 & $\mathrm{C}(14 \mathrm{~A})-\mathrm{C}(8 \mathrm{~A})-\mathrm{P}(1 \mathrm{~A})$ & 121.3(3) \\
\hline $\mathrm{Cl}(16)-\mathrm{C}(8)$ & 1.9882 & $\mathrm{O}(2 \mathrm{~A})-\mathrm{C}(9 \mathrm{~A})-\mathrm{C}(8 \mathrm{~A})$ & $115.6(3)$ \\
\hline $\mathrm{Cl}(17)-\mathrm{C}(9) \# 1$ & 1.7424 & $\mathrm{O}(2 \mathrm{~A})-\mathrm{C}(9 \mathrm{~A})-\mathrm{C}(11 \mathrm{~A})$ & $124.0(3)$ \\
\hline $\mathrm{Cl}(18)-\mathrm{C}(9)$ & 1.8052 & $\mathrm{C}(8 \mathrm{~A})-\mathrm{C}(9 \mathrm{~A})-\mathrm{C}(11 \mathrm{~A})$ & $120.4(3)$ \\
\hline \multirow[t]{2}{*}{$\mathrm{C}(9)-\mathrm{Cl}(17) \# 1$} & 1.7424 & $\mathrm{C}(9 \mathrm{~A})-\mathrm{C}(11 \mathrm{~A})-\mathrm{C}(12 \mathrm{~A})$ & $120.4(4)$ \\
\hline & & $\mathrm{C}(13 \mathrm{~A})-\mathrm{C}(12 \mathrm{~A})-\mathrm{C}(11 \mathrm{~A})$ & 119.6(4) \\
\hline$C(42 A)-C r(1)-C(36 A)$ & $94.05(15)$ & $C(14 \mathrm{~A})-\mathrm{C}(13 \mathrm{~A})-\mathrm{C}(12 \mathrm{~A})$ & $120.5(4)$ \\
\hline $\mathrm{C}(42 \mathrm{~A})-\mathrm{Cr}(1)-\mathrm{C}(30 \mathrm{~A})$ & $97.35(14)$ & $C(13 \mathrm{~A})-\mathrm{C}(14 \mathrm{~A})-\mathrm{C}(8 \mathrm{~A})$ & $120.6(4)$ \\
\hline $\mathrm{C}(36 \mathrm{~A})-\mathrm{Cr}(1)-\mathrm{C}(30 \mathrm{~A})$ & $98.06(14)$ & $C(21 A)-C(15 A)-C(16 A)$ & $118.0(4)$ \\
\hline $\mathrm{C}(42 \mathrm{~A})-\mathrm{Cr}(1)-\mathrm{O}(1 \mathrm{~A})$ & $167.42(12)$ & $\mathrm{C}(21 \mathrm{~A})-\mathrm{C}(15 \mathrm{~A})-\mathrm{P}(2 \mathrm{~A})$ & $120.3(3)$ \\
\hline $\mathrm{C}(36 \mathrm{~A})-\mathrm{Cr}(1)-\mathrm{O}(1 \mathrm{~A})$ & $89.98(12)$ & $\mathrm{C}(16 \mathrm{~A})-\mathrm{C}(15 \mathrm{~A})-\mathrm{P}(2 \mathrm{~A})$ & $121.2(3)$ \\
\hline $\mathrm{C}(30 \mathrm{~A})-\mathrm{Cr}(1)-\mathrm{O}(1 \mathrm{~A})$ & $93.88(11)$ & $\mathrm{O}(3 \mathrm{~A})-\mathrm{C}(16 \mathrm{~A})-\mathrm{C}(18 \mathrm{~A})$ & $124.4(4)$ \\
\hline
\end{tabular}




\begin{tabular}{|c|c|c|c|}
\hline $\mathrm{O}(3 \mathrm{~A})-\mathrm{C}(16 \mathrm{~A})-\mathrm{C}(15 \mathrm{~A})$ & $114.5(3)$ & $\mathrm{P}(1 \mathrm{~B})-\mathrm{Cr}(2)-\mathrm{P}(2 \mathrm{~B})$ & $64.37(4)$ \\
\hline$C(18 \mathrm{~A})-\mathrm{C}(16 \mathrm{~A})-\mathrm{C}(15 \mathrm{~A})$ & $121.0(4)$ & $\mathrm{N}(1 \mathrm{~B})-\mathrm{P}(1 \mathrm{~B})-\mathrm{C}(8 \mathrm{~B})$ & $103.94(17)$ \\
\hline$C(19 A)-C(18 A)-C(16 A)$ & $119.8(4)$ & $\mathrm{N}(1 \mathrm{~B})-\mathrm{P}(1 \mathrm{~B})-\mathrm{C}(3 \mathrm{~B})$ & $104.72(17)$ \\
\hline$C(20 A)-C(19 A)-C(18 A)$ & $120.1(4)$ & $\mathrm{C}(8 \mathrm{~B})-\mathrm{P}(1 \mathrm{~B})-\mathrm{C}(3 \mathrm{~B})$ & $105.26(18)$ \\
\hline$C(19 A)-C(20 A)-C(21 A)$ & $119.8(4)$ & $\mathrm{N}(1 \mathrm{~B})-\mathrm{P}(1 \mathrm{~B})-\mathrm{Cr}(2)$ & $95.94(11)$ \\
\hline$C(15 A)-C(21 A)-C(20 A)$ & 121.3(4) & $\mathrm{C}(8 \mathrm{~B})-\mathrm{P}(1 \mathrm{~B})-\mathrm{Cr}(2)$ & $138.16(13)$ \\
\hline $\mathrm{C}(28 \mathrm{~A})-\mathrm{C}(22 \mathrm{~A})-\mathrm{C}(23 \mathrm{~A})$ & $118.5(4)$ & $\mathrm{C}(3 \mathrm{~B})-\mathrm{P}(1 \mathrm{~B})-\mathrm{Cr}(2)$ & $104.66(13)$ \\
\hline $\mathrm{C}(28 \mathrm{~A})-\mathrm{C}(22 \mathrm{~A})-\mathrm{P}(2 \mathrm{~A})$ & $116.3(3)$ & $\mathrm{N}(1 \mathrm{~B})-\mathrm{P}(2 \mathrm{~B})-\mathrm{C}(15 \mathrm{~B})$ & $105.58(16)$ \\
\hline $\mathrm{C}(23 \mathrm{~A})-\mathrm{C}(22 \mathrm{~A})-\mathrm{P}(2 \mathrm{~A})$ & $125.2(3)$ & $\mathrm{N}(1 \mathrm{~B})-\mathrm{P}(2 \mathrm{~B})-\mathrm{C}(22 \mathrm{~B})$ & $104.19(16)$ \\
\hline $\mathrm{O}(4 \mathrm{~A})-\mathrm{C}(23 \mathrm{~A})-\mathrm{C}(25 \mathrm{~A})$ & $123.8(4)$ & $\mathrm{C}(15 \mathrm{~B})-\mathrm{P}(2 \mathrm{~B})-\mathrm{C}(22 \mathrm{~B})$ & $105.87(18)$ \\
\hline $\mathrm{O}(4 \mathrm{~A})-\mathrm{C}(23 \mathrm{~A})-\mathrm{C}(22 \mathrm{~A})$ & $116.6(3)$ & $\mathrm{N}(1 \mathrm{~B})-\mathrm{P}(2 \mathrm{~B})-\mathrm{Cr}(2)$ & $91.62(11)$ \\
\hline$C(25 A)-C(23 A)-C(22 A)$ & $119.6(4)$ & $\mathrm{C}(15 \mathrm{~B})-\mathrm{P}(2 \mathrm{~B})-\mathrm{Cr}(2)$ & $119.05(13)$ \\
\hline$C(23 A)-C(25 A)-C(26 A)$ & $120.3(4)$ & $\mathrm{C}(22 \mathrm{~B})-\mathrm{P}(2 \mathrm{~B})-\mathrm{Cr}(2)$ & $126.03(14)$ \\
\hline$C(27 A)-C(26 A)-C(25 A)$ & $120.9(4)$ & $\mathrm{C}(2 \mathrm{~B})-\mathrm{O}(1 \mathrm{~B})-\mathrm{C}(1 \mathrm{~B})$ & $115.6(3)$ \\
\hline$C(26 A)-C(27 A)-C(28 A)$ & $119.4(4)$ & $\mathrm{C}(2 \mathrm{~B})-\mathrm{O}(1 \mathrm{~B})-\mathrm{Cr}(2)$ & $127.9(2)$ \\
\hline $\mathrm{C}(27 \mathrm{~A})-\mathrm{C}(28 \mathrm{~A})-\mathrm{C}(22 \mathrm{~A})$ & $121.2(4)$ & $\mathrm{C}(1 \mathrm{~B})-\mathrm{O}(1 \mathrm{~B})-\mathrm{Cr}(2)$ & $115.8(2)$ \\
\hline$C(31 A)-C(30 A)-C(35 A)$ & $114.6(3)$ & $\mathrm{C}(9 \mathrm{~B})-\mathrm{O}(2 \mathrm{~B})-\mathrm{C}(10 \mathrm{~B})$ & $118.7(3)$ \\
\hline $\mathrm{C}(31 \mathrm{~A})-\mathrm{C}(30 \mathrm{~A})-\mathrm{Cr}(1)$ & $125.0(3)$ & $\mathrm{C}(16 \mathrm{~B})-\mathrm{O}(3 \mathrm{~B})-\mathrm{C}(17 \mathrm{~B})$ & $118.4(3)$ \\
\hline $\mathrm{C}(35 \mathrm{~A})-\mathrm{C}(30 \mathrm{~A})-\mathrm{Cr}(1)$ & $120.1(3)$ & $\mathrm{C}(23 \mathrm{~B})-\mathrm{O}(4 \mathrm{~B})-\mathrm{C}(24 \mathrm{~B})$ & 119.3(3) \\
\hline$C(30 A)-C(31 A)-C(32 A)$ & $123.7(4)$ & $\mathrm{C}(29 \mathrm{~B})-\mathrm{N}(1 \mathrm{~B})-\mathrm{P}(1 \mathrm{~B})$ & $121.3(2)$ \\
\hline$C(33 A)-C(32 A)-C(31 A)$ & $119.5(4)$ & $\mathrm{C}(29 \mathrm{~B})-\mathrm{N}(1 \mathrm{~B})-\mathrm{P}(2 \mathrm{~B})$ & $122.6(2)$ \\
\hline $\mathrm{C}(32 \mathrm{~A})-\mathrm{C}(33 \mathrm{~A})-\mathrm{C}(34 \mathrm{~A})$ & $119.5(4)$ & $\mathrm{P}(1 \mathrm{~B})-\mathrm{N}(1 \mathrm{~B})-\mathrm{P}(2 \mathrm{~B})$ & $105.92(17)$ \\
\hline $\mathrm{C}(35 \mathrm{~A})-\mathrm{C}(34 \mathrm{~A})-\mathrm{C}(33 \mathrm{~A})$ & $119.8(4)$ & $\mathrm{C}(7 \mathrm{~B})-\mathrm{C}(2 \mathrm{~B})-\mathrm{O}(1 \mathrm{~B})$ & $123.2(4)$ \\
\hline$C(34 A)-C(35 A)-C(30 A)$ & $122.9(4)$ & $C(7 B)-C(2 B)-C(3 B)$ & $120.5(4)$ \\
\hline$C(41 A)-C(36 A)-C(37 A)$ & $114.6(4)$ & $\mathrm{O}(1 \mathrm{~B})-\mathrm{C}(2 \mathrm{~B})-\mathrm{C}(3 \mathrm{~B})$ & $116.3(4)$ \\
\hline$C(41 A)-C(36 A)-C r(1)$ & $122.2(3)$ & $\mathrm{C}(4 \mathrm{~B})-\mathrm{C}(3 \mathrm{~B})-\mathrm{C}(2 \mathrm{~B})$ & $118.3(4)$ \\
\hline $\mathrm{C}(37 \mathrm{~A})-\mathrm{C}(36 \mathrm{~A})-\mathrm{Cr}(1)$ & 123.1(3) & $\mathrm{C}(4 \mathrm{~B})-\mathrm{C}(3 \mathrm{~B})-\mathrm{P}(1 \mathrm{~B})$ & $124.3(3)$ \\
\hline$C(38 A)-C(37 A)-C(36 A)$ & $122.7(4)$ & $\mathrm{C}(2 \mathrm{~B})-\mathrm{C}(3 \mathrm{~B})-\mathrm{P}(1 \mathrm{~B})$ & $117.4(3)$ \\
\hline$C(39 A)-C(38 A)-C(37 A)$ & $120.5(4)$ & $\mathrm{C}(3 \mathrm{~B})-\mathrm{C}(4 \mathrm{~B})-\mathrm{C}(5 \mathrm{~B})$ & $121.3(4)$ \\
\hline$C(38 A)-C(39 A)-C(40 A)$ & 119.2(4) & $C(6 B)-C(5 B)-C(4 B)$ & $119.0(4)$ \\
\hline$C(39 A)-C(40 A)-C(41 A)$ & $119.4(4)$ & $\mathrm{C}(5 \mathrm{~B})-\mathrm{C}(6 \mathrm{~B})-\mathrm{C}(7 \mathrm{~B})$ & 121.1(4) \\
\hline$C(40 A)-C(41 A)-C(36 A)$ & $123.5(4)$ & $\mathrm{C}(6 \mathrm{~B})-\mathrm{C}(7 \mathrm{~B})-\mathrm{C}(2 \mathrm{~B})$ & $119.8(4)$ \\
\hline$C(47 A)-C(42 A)-C(43 A)$ & $115.2(3)$ & $\mathrm{C}(9 \mathrm{~B})-\mathrm{C}(8 \mathrm{~B})-\mathrm{C}(14 \mathrm{~B})$ & $118.7(4)$ \\
\hline C(47A)-C(42A)-Cr(1) & $121.6(3)$ & C(9B)-C(8B)-P(1B) & $119.9(3)$ \\
\hline C(43A)-C(42A)-Cr(1) & 123.1(3) & $\mathrm{C}(14 \mathrm{~B})-\mathrm{C}(8 \mathrm{~B})-\mathrm{P}(1 \mathrm{~B})$ & 121.3(3) \\
\hline$C(44 A)-C(43 A)-C(42 A)$ & $123.0(4)$ & $\mathrm{O}(2 \mathrm{~B})-\mathrm{C}(9 \mathrm{~B})-\mathrm{C}(8 \mathrm{~B})$ & $115.5(3)$ \\
\hline$C(43 \mathrm{~A})-\mathrm{C}(44 \mathrm{~A})-\mathrm{C}(45 \mathrm{~A})$ & $119.8(4)$ & $\mathrm{O}(2 \mathrm{~B})-\mathrm{C}(9 \mathrm{~B})-\mathrm{C}(11 \mathrm{~B})$ & 124.3(4) \\
\hline$C(44 A)-C(45 A)-C(46 A)$ & 119.3(4) & C(8B)-C(9B)-C(11B) & 120.1(4) \\
\hline$C(47 A)-C(46 A)-C(45 A)$ & $119.8(4)$ & $\mathrm{C}(12 \mathrm{~B})-\mathrm{C}(11 \mathrm{~B})-\mathrm{C}(9 \mathrm{~B})$ & $120.2(4)$ \\
\hline$C(46 \mathrm{~A})-\mathrm{C}(47 \mathrm{~A})-\mathrm{C}(42 \mathrm{~A})$ & $122.8(4)$ & $\mathrm{C}(11 \mathrm{~B})-\mathrm{C}(12 \mathrm{~B})-\mathrm{C}(13 \mathrm{~B})$ & $120.3(4)$ \\
\hline $\mathrm{C}(42 \mathrm{~B})-\mathrm{Cr}(2)-\mathrm{C}(36 \mathrm{~B})$ & $92.86(15)$ & $\mathrm{C}(14 \mathrm{~B})-\mathrm{C}(13 \mathrm{~B})-\mathrm{C}(12 \mathrm{~B})$ & 119.3(4) \\
\hline $\mathrm{C}(42 \mathrm{~B})-\mathrm{Cr}(2)-\mathrm{C}(30 \mathrm{~B})$ & $96.62(15)$ & C(13B)-C(14B)-C(8B) & $121.2(4)$ \\
\hline $\mathrm{C}(36 \mathrm{~B})-\mathrm{Cr}(2)-\mathrm{C}(30 \mathrm{~B})$ & $99.25(15)$ & $C(21 B)-C(15 B)-C(16 B)$ & $117.6(4)$ \\
\hline $\mathrm{C}(42 \mathrm{~B})-\mathrm{Cr}(2)-\mathrm{O}(1 \mathrm{~B})$ & $168.94(13)$ & $\mathrm{C}(21 \mathrm{~B})-\mathrm{C}(15 \mathrm{~B})-\mathrm{P}(2 \mathrm{~B})$ & $121.2(3)$ \\
\hline $\mathrm{C}(36 \mathrm{~B})-\mathrm{Cr}(2)-\mathrm{O}(1 \mathrm{~B})$ & $90.87(13)$ & $\mathrm{C}(16 \mathrm{~B})-\mathrm{C}(15 \mathrm{~B})-\mathrm{P}(2 \mathrm{~B})$ & $121.0(3)$ \\
\hline $\mathrm{C}(30 \mathrm{~B})-\mathrm{Cr}(2)-\mathrm{O}(1 \mathrm{~B})$ & $93.04(12)$ & $\mathrm{O}(3 \mathrm{~B})-\mathrm{C}(16 \mathrm{~B})-\mathrm{C}(18 \mathrm{~B})$ & 124.1(4) \\
\hline $\mathrm{C}(42 \mathrm{~B})-\mathrm{Cr}(2)-\mathrm{P}(1 \mathrm{~B})$ & $95.52(11)$ & $\mathrm{O}(3 \mathrm{~B})-\mathrm{C}(16 \mathrm{~B})-\mathrm{C}(15 \mathrm{~B})$ & $115.1(3)$ \\
\hline $\mathrm{C}(36 \mathrm{~B})-\mathrm{Cr}(2)-\mathrm{P}(1 \mathrm{~B})$ & $97.63(11)$ & $\mathrm{C}(18 \mathrm{~B})-\mathrm{C}(16 \mathrm{~B})-\mathrm{C}(15 \mathrm{~B})$ & $120.8(4)$ \\
\hline $\mathrm{C}(30 \mathrm{~B})-\mathrm{Cr}(2)-\mathrm{P}(1 \mathrm{~B})$ & $158.64(12)$ & $\mathrm{C}(16 \mathrm{~B})-\mathrm{C}(18 \mathrm{~B})-\mathrm{C}(19 \mathrm{~B})$ & $119.7(4)$ \\
\hline $\mathrm{O}(1 \mathrm{~B})-\mathrm{Cr}(2)-\mathrm{P}(1 \mathrm{~B})$ & $73.66(7)$ & $C(20 B)-C(19 B)-C(18 B)$ & $120.2(4)$ \\
\hline $\mathrm{C}(42 \mathrm{~B})-\mathrm{Cr}(2)-\mathrm{P}(2 \mathrm{~B})$ & $83.95(11)$ & $C(19 B)-C(20 B)-C(21 B)$ & $120.4(4)$ \\
\hline $\mathrm{C}(36 \mathrm{~B})-\mathrm{Cr}(2)-\mathrm{P}(2 \mathrm{~B})$ & $161.18(11)$ & $C(15 B)-C(21 B)-C(20 B)$ & $121.3(4)$ \\
\hline $\mathrm{C}(30 \mathrm{~B})-\mathrm{Cr}(2)-\mathrm{P}(2 \mathrm{~B})$ & $99.55(11)$ & $\mathrm{C}(23 \mathrm{~B})-\mathrm{C}(22 \mathrm{~B})-\mathrm{C}(28 \mathrm{~B})$ & $117.5(4)$ \\
\hline $\mathrm{O}(1 \mathrm{~B})-\mathrm{Cr}(2)-\mathrm{P}(2 \mathrm{~B})$ & $89.16(7)$ & $\mathrm{C}(23 \mathrm{~B})-\mathrm{C}(22 \mathrm{~B})-\mathrm{P}(2 \mathrm{~B})$ & 124.4(3) \\
\hline
\end{tabular}




$\begin{array}{llll}\mathrm{C}(28 \mathrm{~B})-\mathrm{C}(22 \mathrm{~B})-\mathrm{P}(2 \mathrm{~B}) & 118.1(3) & \mathrm{C}(38 \mathrm{~B})-\mathrm{C}(39 \mathrm{~B})-\mathrm{C}(40 \mathrm{~B}) & 119.3(4) \\ \mathrm{O}(4 \mathrm{~B})-\mathrm{C}(23 \mathrm{~B})-\mathrm{C}(22 \mathrm{~B}) & 116.2(4) & \mathrm{C}(39 \mathrm{~B})-\mathrm{C}(40 \mathrm{~B})-\mathrm{C}(41 \mathrm{~B}) & 119.5(4) \\ \mathrm{O}(4 \mathrm{~B})-\mathrm{C}(23 \mathrm{~B})-\mathrm{C}(25 \mathrm{~B}) & 122.9(4) & \mathrm{C}(40 \mathrm{~B})-\mathrm{C}(41 \mathrm{~B})-\mathrm{C}(36 \mathrm{~B}) & 123.4(4) \\ \mathrm{C}(22 \mathrm{~B})-\mathrm{C}(23 \mathrm{~B})-\mathrm{C}(25 \mathrm{~B}) & 120.8(4) & \mathrm{C}(47 \mathrm{~B})-\mathrm{C}(42 \mathrm{~B})-\mathrm{C}(43 \mathrm{~B}) & 115.0(4) \\ \mathrm{C}(26 \mathrm{~B})-\mathrm{C}(25 \mathrm{~B})-\mathrm{C}(23 \mathrm{~B}) & 119.2(4) & \mathrm{C}(47 \mathrm{~B})-\mathrm{C}(42 \mathrm{~B})-\mathrm{Cr}(2) & 123.5(3) \\ \mathrm{C}(25 \mathrm{~B})-\mathrm{C}(26 \mathrm{~B})-\mathrm{C}(27 \mathrm{~B}) & 120.9(4) & \mathrm{C}(43 \mathrm{~B})-\mathrm{C}(42 \mathrm{~B})-\mathrm{Cr}(2) & 121.5(3) \\ \mathrm{C}(28 \mathrm{~B})-\mathrm{C}(27 \mathrm{~B})-\mathrm{C}(26 \mathrm{~B}) & 119.3(4) & \mathrm{C}(44 \mathrm{~B})-\mathrm{C}(43 \mathrm{~B})-\mathrm{C}(42 \mathrm{~B}) & 122.7(4) \\ \mathrm{C}(27 \mathrm{~B})-\mathrm{C}(28 \mathrm{~B})-\mathrm{C}(22 \mathrm{~B}) & 122.2(4) & \mathrm{C}(43 \mathrm{~B})-\mathrm{C}(44 \mathrm{~B})-\mathrm{C}(45 \mathrm{~B}) & 120.9(4) \\ \mathrm{C}(31 \mathrm{~B})-\mathrm{C}(30 \mathrm{~B})-\mathrm{C}(35 \mathrm{~B}) & 114.0(4) & \mathrm{C}(44 \mathrm{~B})-\mathrm{C}(45 \mathrm{~B})-\mathrm{C}(46 \mathrm{~B}) & 118.1(4) \\ \mathrm{C}(31 \mathrm{~B})-\mathrm{C}(30 \mathrm{~B})-\mathrm{Cr}(2) & 123.3(3) & \mathrm{C}(47 \mathrm{~B})-\mathrm{C}(46 \mathrm{~B})-\mathrm{C}(45 \mathrm{~B}) & 120.7(4) \\ \mathrm{C}(35 \mathrm{~B})-\mathrm{C}(30 \mathrm{~B})-\mathrm{Cr}(2) & 122.6(3) & \mathrm{C}(46 \mathrm{~B})-\mathrm{C}(47 \mathrm{~B})-\mathrm{C}(42 \mathrm{~B}) & 122.6(4) \\ \mathrm{C}(30 \mathrm{~B})-\mathrm{C}(31 \mathrm{~B})-\mathrm{C}(32 \mathrm{~B}) & 123.4(4) & \mathrm{Cl}(1)-\mathrm{C}(1)-\mathrm{Cl}(2) & 113.6(3) \\ \mathrm{C}(33 \mathrm{~B})-\mathrm{C}(32 \mathrm{~B})-\mathrm{C}(31 \mathrm{~B}) & 119.4(4) & \mathrm{Cl}(4)-\mathrm{C}(2)-\mathrm{Cl}(3) & 110.5(2) \\ \mathrm{C}(34 \mathrm{~B})-\mathrm{C}(33 \mathrm{~B})-\mathrm{C}(32 \mathrm{~B}) & 119.0(4) & \mathrm{Cl}(5)-\mathrm{C}(3)-\mathrm{Cl}(6) & 110.7(3) \\ \mathrm{C}(33 \mathrm{~B})-\mathrm{C}(34 \mathrm{~B})-\mathrm{C}(35 \mathrm{~B}) & 121.0(4) & \mathrm{Cl}(8)-\mathrm{C}(4)-\mathrm{Cl}(7) & 111.8(3) \\ \mathrm{C}(34 \mathrm{~B})-\mathrm{C}(35 \mathrm{~B})-\mathrm{C}(30 \mathrm{~B}) & 123.0(4) & \mathrm{Cl}(9)-\mathrm{C}(5)-\mathrm{Cl}(10) & 113.9(4) \\ \mathrm{C}(37 \mathrm{~B})-\mathrm{C}(36 \mathrm{~B})-\mathrm{C}(41 \mathrm{~B}) & 114.5(4) & \mathrm{Cl}(12)-\mathrm{C}(6)-\mathrm{Cl}(11) & 120.1(7) \\ \mathrm{C}(37 \mathrm{~B})-\mathrm{C}(36 \mathrm{~B})-\mathrm{Cr}(2) & 123.5(3) & \mathrm{Cl}(14)-\mathrm{C}(7)-\mathrm{Cl}(13) & 101.340(4) \\ \mathrm{C}(41 \mathrm{~B})-\mathrm{C}(36 \mathrm{~B})-\mathrm{Cr}(2) & 122.0(3) & \mathrm{Cl}(15)-\mathrm{C}(8)-\mathrm{Cl}(16) & 130.592(3) \\ \mathrm{C}(38 \mathrm{~B})-\mathrm{C}(37 \mathrm{~B})-\mathrm{C}(36 \mathrm{~B}) & 123.7(4) & \mathrm{Cl}(17) \# 1-\mathrm{C}(9)-\mathrm{Cl}(18) & 115.854(3) \\ \mathrm{C}(39 \mathrm{~B})-\mathrm{C}(38 \mathrm{~B})-\mathrm{C}(37 \mathrm{~B}) & 119.6(4) & & \end{array}$

Symmetry transformations used to generate equivalent atoms:

\#1 - x+1,-y+1,-z+1 
Table 11. Anisotropic displacement parameters $\left(\AA^{2} \times 10^{4}\right)$ for 3 (CCDC 216617). The anisotropic displacement factor exponent takes the form: $-2 \square^{2}\left[h^{2} a^{* 2} U^{11}+\ldots+2 h k^{*} b^{*}\right.$ $\left.\mathbf{U}^{12}\right]$

\begin{tabular}{|c|c|c|c|c|c|c|}
\hline & $\mathrm{U}^{11}$ & $\mathrm{U}^{22}$ & $\mathrm{U}^{33}$ & $\mathrm{U}^{23}$ & $\mathrm{U}^{13}$ & $\mathrm{U}^{12}$ \\
\hline $\operatorname{Cr}(1)$ & $110(3)$ & $172(4)$ & $146(3)$ & $18(3)$ & $14(3)$ & $77(3)$ \\
\hline $\mathrm{P}(1 \mathrm{~A})$ & $112(5)$ & $168(6)$ & $156(6)$ & $16(5)$ & $15(4)$ & $70(4)$ \\
\hline $\mathrm{P}(2 \mathrm{~A})$ & $126(5)$ & $172(6)$ & $160(6)$ & $26(5)$ & $9(4)$ & $73(5)$ \\
\hline $\mathrm{O}(1 \mathrm{~A})$ & 101(13) & 194(16) & $172(15)$ & $18(12)$ & $36(11)$ & $66(12)$ \\
\hline $\mathrm{O}(2 \mathrm{~A})$ & $156(15)$ & $233(17)$ & 261(17) & 119(13) & $68(13)$ & $95(13)$ \\
\hline $\mathrm{O}(3 \mathrm{~A})$ & 181(15) & 231(17) & 279(17) & 93(13) & $36(13)$ & $119(13)$ \\
\hline $\mathrm{O}(4 \mathrm{~A})$ & $220(16)$ & $345(18)$ & 139(15) & $68(13)$ & $31(12)$ & $109(14)$ \\
\hline $\mathrm{N}(1 \mathrm{~A})$ & $170(17)$ & 188(19) & $106(17)$ & $23(14)$ & $29(14)$ & $110(15)$ \\
\hline $\mathrm{C}(1 \mathrm{~A})$ & $110(20)$ & $240(30)$ & $390(30)$ & $-10(20)$ & $76(19)$ & 55(19) \\
\hline $\mathrm{C}(2 \mathrm{~A})$ & $190(20)$ & $150(20)$ & $130(20)$ & 1(17) & $-18(17)$ & $71(18)$ \\
\hline $\mathrm{C}(3 \mathrm{~A})$ & $160(20)$ & $200(20)$ & $140(20)$ & $23(18)$ & $24(17)$ & $97(18)$ \\
\hline $\mathrm{C}(4 \mathrm{~A})$ & $180(20)$ & $260(30)$ & $340(30)$ & $-80(20)$ & $10(20)$ & $90(20)$ \\
\hline$C(5 \mathrm{~A})$ & $290(30)$ & $240(30)$ & $520(30)$ & $-150(20)$ & $20(20)$ & $90(20)$ \\
\hline$C(6 A)$ & $170(20)$ & $210(30)$ & $410(30)$ & $-50(20)$ & $0(20)$ & $-10(20)$ \\
\hline $\mathrm{C}(7 \mathrm{~A})$ & $120(20)$ & $210(20)$ & $240(20)$ & $-9(19)$ & $32(18)$ & $15(18)$ \\
\hline $\mathrm{C}(8 \mathrm{~A})$ & 93(19) & $190(20)$ & $140(20)$ & $4(17)$ & $-21(16)$ & $91(17)$ \\
\hline $\mathrm{C}(9 \mathrm{~A})$ & $110(20)$ & $200(20)$ & $160(20)$ & $-3(18)$ & $24(17)$ & $69(18)$ \\
\hline$C(10 A)$ & $210(20)$ & $340(30)$ & $410(30)$ & $170(20)$ & $100(20)$ & $90(20)$ \\
\hline $\mathrm{C}(11 \mathrm{~A})$ & $200(20)$ & $190(20)$ & $190(20)$ & $35(18)$ & 7(18) & 119(19) \\
\hline $\mathrm{C}(12 \mathrm{~A})$ & $130(20)$ & $270(30)$ & $220(20)$ & $-15(19)$ & $-3(18)$ & 123(19) \\
\hline$C(13 A)$ & $120(20)$ & $230(20)$ & $230(20)$ & 8(19) & $48(18)$ & 61(18) \\
\hline $\mathrm{C}(14 \mathrm{~A})$ & $160(20)$ & $200(20)$ & $160(20)$ & 19(18) & 11(17) & $81(18)$ \\
\hline$C(15 A)$ & $210(20)$ & $200(20)$ & $130(20)$ & $-11(18)$ & $32(17)$ & 72(19) \\
\hline$C(16 A)$ & $170(20)$ & $220(20)$ & $160(20)$ & $-12(18)$ & $10(17)$ & $94(19)$ \\
\hline $\mathrm{C}(17 \mathrm{~A})$ & $270(20)$ & $320(30)$ & $300(30)$ & $80(20)$ & $10(20)$ & $190(20)$ \\
\hline $\mathrm{C}(18 \mathrm{~A})$ & $250(20)$ & $230(30)$ & $290(30)$ & $80(20)$ & $90(20)$ & $120(20)$ \\
\hline $\mathrm{C}(19 \mathrm{~A})$ & $250(20)$ & $190(20)$ & $370(30)$ & $50(20)$ & $110(20)$ & $40(20)$ \\
\hline$C(20 A)$ & $180(20)$ & $260(30)$ & $310(30)$ & $20(20)$ & $80(20)$ & $60(20)$ \\
\hline$C(21 A)$ & $170(20)$ & $210(20)$ & $220(20)$ & $14(19)$ & $48(18)$ & $84(19)$ \\
\hline$C(22 A)$ & $170(20)$ & $170(20)$ & $180(20)$ & 4(18) & $-35(17)$ & $108(18)$ \\
\hline$C(23 A)$ & $240(20)$ & $230(20)$ & $220(20)$ & $50(20)$ & $-10(19)$ & $120(20)$ \\
\hline$C(24 A)$ & $410(30)$ & $460(30)$ & $190(30)$ & $70(20)$ & $70(20)$ & $200(30)$ \\
\hline $\mathrm{C}(25 \mathrm{~A})$ & $330(30)$ & $380(30)$ & $190(20)$ & $50(20)$ & $-50(20)$ & $110(20)$ \\
\hline$C(26 A)$ & $330(30)$ & $350(30)$ & $270(30)$ & $-10(20)$ & $-190(20)$ & $60(20)$ \\
\hline$C(27 A)$ & $160(20)$ & $270(30)$ & $320(30)$ & $30(20)$ & $-30(20)$ & $50(20)$ \\
\hline$C(28 A)$ & $180(20)$ & $160(20)$ & $240(20)$ & $32(18)$ & $-17(18)$ & $78(18)$ \\
\hline$C(29 A)$ & $210(20)$ & $330(30)$ & $130(20)$ & $-20(19)$ & 8(18) & $140(20)$ \\
\hline$C(30 A)$ & 100(19) & $130(20)$ & $140(20)$ & 23(17) & $-7(16)$ & $38(16)$ \\
\hline$C(31 \mathrm{~A})$ & $190(20)$ & $180(20)$ & $180(20)$ & 12(18) & $-34(18)$ & $76(18)$ \\
\hline$C(32 A)$ & $140(20)$ & $230(20)$ & $230(20)$ & $-49(19)$ & $48(18)$ & $56(19)$ \\
\hline$C(33 A)$ & $120(20)$ & $230(20)$ & $340(30)$ & $0(20)$ & 13(19) & 90(19) \\
\hline $\mathrm{C}(34 \mathrm{~A})$ & $220(20)$ & $240(30)$ & $250(30)$ & $50(20)$ & $-9(19)$ & $150(20)$ \\
\hline$C(35 A)$ & $180(20)$ & $230(20)$ & $240(20)$ & 14(19) & $68(18)$ & $100(19)$ \\
\hline$C(36 A)$ & 78(19) & $230(20)$ & $160(20)$ & $15(18)$ & $30(16)$ & $103(17)$ \\
\hline $\mathrm{C}(37 \mathrm{~A})$ & $120(20)$ & $290(30)$ & $180(20)$ & 42(19) & $16(17)$ & 132(19) \\
\hline $\mathrm{C}(38 \mathrm{~A})$ & $140(20)$ & $290(30)$ & $230(20)$ & $100(20)$ & $31(18)$ & $130(19)$ \\
\hline$C(39 A)$ & $170(20)$ & $440(30)$ & $180(20)$ & $110(20)$ & $65(18)$ & $200(20)$ \\
\hline
\end{tabular}




\begin{tabular}{|c|c|c|c|c|c|c|}
\hline $\mathrm{C}(40 \mathrm{~A})$ & $160(20)$ & $370(30)$ & $180(20)$ & $0(20)$ & $12(18)$ & $160(20)$ \\
\hline $\mathrm{C}(41 \mathrm{~A})$ & $180(20)$ & $250(20)$ & $220(20)$ & $10(20)$ & $23(18)$ & $134(19)$ \\
\hline $\mathrm{C}(42 \mathrm{~A})$ & $150(20)$ & $180(20)$ & $110(20)$ & $-27(17)$ & 19(16) & $53(18)$ \\
\hline $\mathrm{C}(43 \mathrm{~A})$ & $130(20)$ & $180(20)$ & $180(20)$ & $-28(18)$ & $2(17)$ & $66(18)$ \\
\hline $\mathrm{C}(44 \mathrm{~A})$ & $230(20)$ & $200(20)$ & $280(30)$ & $-3(19)$ & $42(19)$ & $80(20)$ \\
\hline $\mathrm{C}(45 \mathrm{~A})$ & $170(20)$ & $250(30)$ & $360(30)$ & $-50(20)$ & $0(20)$ & $20(20)$ \\
\hline$C(46 \mathrm{~A})$ & $160(20)$ & $330(30)$ & $270(30)$ & $-40(20)$ & $-32(19)$ & $140(20)$ \\
\hline$C(47 A)$ & $210(20)$ & $190(20)$ & $180(20)$ & 1(18) & $-2(18)$ & $94(19)$ \\
\hline $\mathrm{Cr}(2)$ & $138(3)$ & $262(4)$ & $147(4)$ & $25(3)$ & $7(3)$ & $90(3)$ \\
\hline $\mathrm{P}(1 \mathrm{~B})$ & $154(5)$ & $240(6)$ & $145(6)$ & $3(5)$ & $-5(4)$ & $90(5)$ \\
\hline $\mathrm{P}(2 \mathrm{~B})$ & $142(5)$ & $230(6)$ & $148(6)$ & $19(5)$ & $-3(4)$ & $79(5)$ \\
\hline $\mathrm{O}(1 \mathrm{~B})$ & $141(14)$ & $303(18)$ & $206(16)$ & $50(13)$ & $46(12)$ & $111(13)$ \\
\hline $\mathrm{O}(2 \mathrm{~B})$ & $228(16)$ & $420(20)$ & $137(16)$ & $25(14)$ & $-8(13)$ & $156(15)$ \\
\hline $\mathrm{O}(3 \mathrm{~B})$ & $162(15)$ & $417(19)$ & $180(16)$ & $-59(14)$ & $-61(12)$ & $146(14)$ \\
\hline $\mathrm{O}(4 \mathrm{~B})$ & $295(17)$ & $289(18)$ & $205(17)$ & $105(14)$ & $85(13)$ & $125(14)$ \\
\hline $\mathrm{N}(1 \mathrm{~B})$ & $149(17)$ & 203(19) & $110(17)$ & $3(14)$ & $-10(14)$ & $58(15)$ \\
\hline $\mathrm{C}(1 \mathrm{~B})$ & $210(20)$ & $450(30)$ & $450(30)$ & $10(20)$ & $100(20)$ & $160(20)$ \\
\hline $\mathrm{C}(2 \mathrm{~B})$ & $270(20)$ & $240(30)$ & $150(20)$ & $17(19)$ & $-10(19)$ & $110(20)$ \\
\hline $\mathrm{C}(3 \mathrm{~B})$ & $250(20)$ & $270(30)$ & $130(20)$ & $34(19)$ & $4(18)$ & $130(20)$ \\
\hline $\mathrm{C}(4 \mathrm{~B})$ & $250(20)$ & $250(30)$ & $180(20)$ & $-62(19)$ & $-39(19)$ & $70(20)$ \\
\hline $\mathrm{C}(5 \mathrm{~B})$ & $380(30)$ & $270(30)$ & $290(30)$ & $-50(20)$ & $-40(20)$ & $100(20)$ \\
\hline $\mathrm{C}(6 \mathrm{~B})$ & $510(30)$ & $240(30)$ & $280(30)$ & $-50(20)$ & $20(20)$ & $180(20)$ \\
\hline $\mathrm{C}(7 \mathrm{~B})$ & $350(30)$ & $370(30)$ & $250(30)$ & $40(20)$ & $70(20)$ & $230(20)$ \\
\hline $\mathrm{C}(8 \mathrm{~B})$ & $170(20)$ & $180(20)$ & $170(20)$ & $-10(18)$ & $-38(17)$ & $57(18)$ \\
\hline $\mathrm{C}(9 \mathrm{~B})$ & $210(20)$ & $210(20)$ & $190(20)$ & $35(19)$ & $22(18)$ & $76(19)$ \\
\hline$C(10 B)$ & $290(30)$ & $510(30)$ & $170(20)$ & $0(20)$ & $10(20)$ & $170(20)$ \\
\hline $\mathrm{C}(11 \mathrm{~B})$ & $200(20)$ & $280(30)$ & $210(20)$ & $40(20)$ & $-34(19)$ & $60(20)$ \\
\hline$C(12 B)$ & $110(20)$ & $310(30)$ & $340(30)$ & $60(20)$ & $-13(19)$ & $48(19)$ \\
\hline$C(13 B)$ & $160(20)$ & $320(30)$ & $270(30)$ & $50(20)$ & $36(19)$ & $100(20)$ \\
\hline$C(14 B)$ & $210(20)$ & $240(20)$ & $200(20)$ & $16(19)$ & $-14(19)$ & $78(19)$ \\
\hline$C(15 B)$ & $160(20)$ & $220(20)$ & $130(20)$ & $24(18)$ & $21(17)$ & $67(18)$ \\
\hline$C(16 B)$ & $140(20)$ & $210(20)$ & $200(20)$ & $30(19)$ & $33(18)$ & $47(18)$ \\
\hline$C(17 B)$ & $190(20)$ & $340(30)$ & $280(30)$ & $-10(20)$ & $-75(19)$ & $100(20)$ \\
\hline $\mathrm{C}(18 \mathrm{~B})$ & $210(20)$ & $240(30)$ & $190(20)$ & $0(19)$ & $-29(18)$ & $38(19)$ \\
\hline$C(19 B)$ & $290(30)$ & $240(30)$ & $240(30)$ & $-60(20)$ & $40(20)$ & $80(20)$ \\
\hline $\mathrm{C}(20 \mathrm{~B})$ & $170(20)$ & $210(20)$ & $270(30)$ & $-10(20)$ & $34(19)$ & $82(19)$ \\
\hline$C(21 B)$ & $130(20)$ & $200(20)$ & $190(20)$ & $51(18)$ & $3(17)$ & $43(18)$ \\
\hline $\mathrm{C}(22 \mathrm{~B})$ & $150(20)$ & $180(20)$ & $190(20)$ & $23(18)$ & $-32(17)$ & $57(18)$ \\
\hline$C(23 B)$ & $190(20)$ & $260(30)$ & $210(20)$ & $20(20)$ & $-27(19)$ & $80(20)$ \\
\hline$C(24 B)$ & $370(30)$ & $490(30)$ & $220(30)$ & $150(20)$ & $90(20)$ & $160(20)$ \\
\hline$C(25 B)$ & $270(30)$ & $370(30)$ & $260(30)$ & $110(20)$ & $-20(20)$ & $110(20)$ \\
\hline$C(26 B)$ & $270(30)$ & $320(30)$ & $490(30)$ & $90(20)$ & $-30(20)$ & $200(20)$ \\
\hline $\mathrm{C}(27 \mathrm{~B})$ & $250(20)$ & $320(30)$ & $350(30)$ & $30(20)$ & $20(20)$ & $160(20)$ \\
\hline $\mathrm{C}(28 \mathrm{~B})$ & $180(20)$ & $250(30)$ & $210(20)$ & $34(19)$ & $-32(18)$ & $91(19)$ \\
\hline$C(29 B)$ & $170(20)$ & $260(30)$ & $230(20)$ & $10(20)$ & $-11(18)$ & $59(19)$ \\
\hline$C(30 B)$ & $160(20)$ & $270(30)$ & $160(20)$ & $5(19)$ & $49(17)$ & $118(19)$ \\
\hline$C(31 B)$ & $190(20)$ & $460(30)$ & $260(30)$ & $90(20)$ & $0(20)$ & $110(20)$ \\
\hline$C(32 B)$ & $190(20)$ & $490(30)$ & $240(30)$ & $120(20)$ & $40(20)$ & $60(20)$ \\
\hline$C(33 B)$ & $150(20)$ & $370(30)$ & $270(30)$ & $-30(20)$ & $-30(19)$ & $100(20)$ \\
\hline$C(34 B)$ & $250(20)$ & $250(30)$ & $230(20)$ & $-30(20)$ & $-44(19)$ & $150(20)$ \\
\hline$C(35 B)$ & $200(20)$ & $220(20)$ & $230(20)$ & $-1(19)$ & $7(18)$ & $108(19)$ \\
\hline$C(36 \mathrm{~B})$ & $110(20)$ & $300(30)$ & $170(20)$ & $33(19)$ & $48(17)$ & $108(19)$ \\
\hline $\mathrm{C}(37 \mathrm{~B})$ & $190(20)$ & $240(20)$ & $180(20)$ & $33(19)$ & $45(18)$ & $122(19)$ \\
\hline
\end{tabular}




\begin{tabular}{lcccrrr}
$\mathrm{C}(38 \mathrm{~B})$ & $200(20)$ & $280(30)$ & $160(20)$ & $-13(19)$ & $26(18)$ & $100(20)$ \\
$\mathrm{C}(39 \mathrm{~B})$ & $180(20)$ & $390(30)$ & $170(20)$ & $100(20)$ & $62(18)$ & $170(20)$ \\
$\mathrm{C}(40 \mathrm{~B})$ & $160(20)$ & $240(30)$ & $270(30)$ & $40(20)$ & $18(19)$ & $73(19)$ \\
$\mathrm{C}(41 \mathrm{~B})$ & $160(20)$ & $310(30)$ & $150(20)$ & $-18(19)$ & $-22(17)$ & $100(20)$ \\
$\mathrm{C}(42 \mathrm{~B})$ & $160(20)$ & $270(30)$ & $120(20)$ & $23(18)$ & $28(17)$ & $72(19)$ \\
$\mathrm{C}(43 \mathrm{~B})$ & $160(20)$ & $230(20)$ & $150(20)$ & $20(18)$ & $-31(17)$ & $67(19)$ \\
$\mathrm{C}(44 \mathrm{~B})$ & $190(20)$ & $340(30)$ & $210(20)$ & $0(20)$ & $18(19)$ & $120(20)$ \\
$\mathrm{C}(45 \mathrm{~B})$ & $290(20)$ & $220(30)$ & $260(30)$ & $0(20)$ & $40(20)$ & $120(20)$ \\
$\mathrm{C}(46 \mathrm{~B})$ & $240(20)$ & $290(30)$ & $270(30)$ & $-70(20)$ & $-30(20)$ & $20(20)$ \\
$\mathrm{C}(47 \mathrm{~B})$ & $140(20)$ & $320(30)$ & $230(20)$ & $-30(20)$ & $17(18)$ & $60(20)$ \\
& & & & & \\
$\mathrm{Cl}(1)$ & $446(8)$ & $744(11)$ & $684(10)$ & $2(8)$ & $113(7)$ & $264(8)$ \\
$\mathrm{Cl}(2)$ & $543(9)$ & $814(12)$ & $773(12)$ & $-192(9)$ & $209(8)$ & $219(9)$ \\
$\mathrm{C}(1)$ & $450(40)$ & $1740(80)$ & $1500(70)$ & $-1140(60)$ & $250(40)$ & $150(50)$ \\
& & & & & \\
$\mathrm{Cl}(3)$ & $455(7)$ & $402(7)$ & $314(7)$ & $3(6)$ & $62(6)$ & $254(6)$ \\
$\mathrm{Cl}(4)$ & $309(6)$ & $395(7)$ & $438(8)$ & $49(6)$ & $103(6)$ & $147(6)$ \\
$\mathrm{C}(2)$ & $190(20)$ & $270(30)$ & $430(30)$ & $60(20)$ & $40(20)$ & $130(20)$ \\
& & & & & \\
$\mathrm{Cl}(5)$ & $643(11)$ & $1161(15)$ & $765(12)$ & $68(11)$ & $280(9)$ & $260(11)$ \\
$\mathrm{Cl}(6)$ & $1023(14)$ & $894(14)$ & $932(14)$ & $-94(11)$ & $-132(11)$ & $528(12)$ \\
$\mathrm{C}(3)$ & $1590(70)$ & $1420(70)$ & $530(40)$ & $300(40)$ & $290(40)$ & $1280(60)$ \\
& & & & & \\
$\mathrm{Cl}(7)$ & $719(11)$ & $495(10)$ & $1169(15)$ & $39(10)$ & $313(10)$ & $200(9)$ \\
$\mathrm{Cl}(8)$ & $611(9)$ & $496(9)$ & $467(9)$ & $-129(7)$ & $1(7)$ & $99(7)$ \\
$\mathrm{C}(4)$ & $800(40)$ & $450(40)$ & $360(30)$ & $160(30)$ & $60(30)$ & $160(30)$ \\
& & & & & \\
\hline & & & & &
\end{tabular}




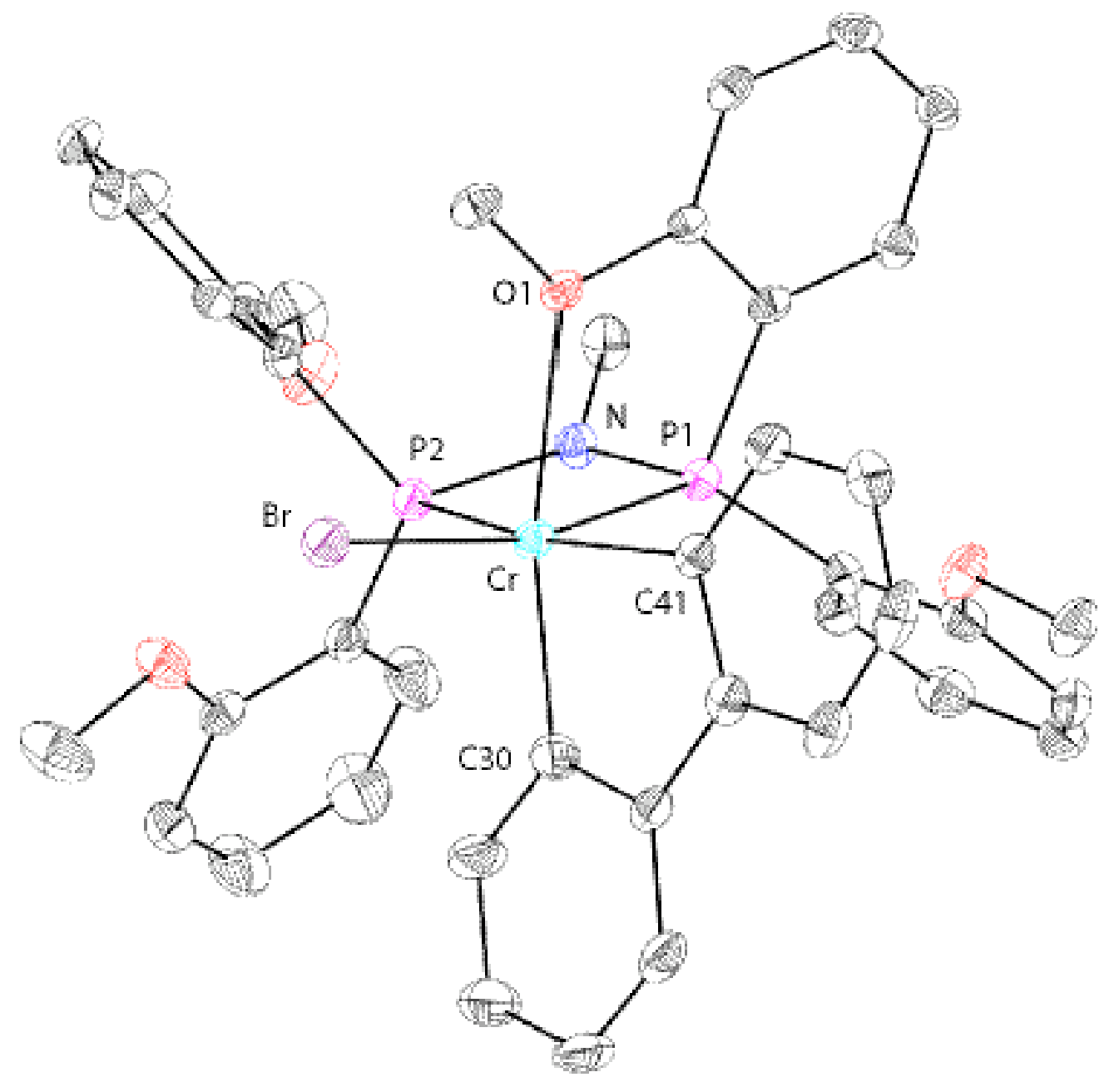

Figure 16. Structural drawing of 4 with thermal ellipsoids at the $50 \%$ probability level. 
Table 12. Crystal data and structure refinement for 4 (CCDC 208405).

Empirical formula

Formula weight

Crystallization Solvent

Crystal Habit

Crystal size

Crystal color

Preliminary Photos

Type of diffractometer

Wavelength

Data Collection Temperature

$\square$ range for 16724 reflections used

in lattice determination

Unit cell dimensions

Volume

Z

Crystal system

Space group

Density (calculated)

$\mathrm{F}(000)$

$\square$ range for data collection

Completeness to $\square=28.29^{\circ}$

Index ranges

Data collection scan type

Reflections collected

Independent reflections

Absorption coefficient

Absorption correction

Max. and min. transmission
$\mathrm{C}_{41} \mathrm{H}_{39} \mathrm{NO}_{4} \mathrm{P}_{2} \mathrm{BrCr} \cdot \mathrm{CH}_{2} \mathrm{Cl}_{2}$

888.51

Dichloromethane/petroleum ether

Fragment

$0.43 \times 0.19 \times 0.03 \mathrm{~mm}^{3}$

Green

\section{Data Collection}

Rotation

Bruker SMART 1000

$0.71073 \AA$ MoK $\square$

98(2) K

2.21 to $27.85^{\circ}$

$a=9.9167(6) \AA$

$\mathrm{b}=11.9935(8) \AA$

$\square=77.3710(10)^{\circ}$

$\square=76.3280(10)^{\circ}$

$c=17.9725(11) \AA$

$\square=70.6700(10)^{\circ}$

1936.8(2) $\AA^{3}$

2

Triclinic

P-1

$1.524 \mathrm{Mg} / \mathrm{m}^{3}$

910

1.82 to $28.29^{\circ}$

$91.9 \%$

$-12 \leq \mathrm{h} \leq 13,-15 \leq \mathrm{k} \leq 15,-23 \leq 1 \leq 23$

$\square$ scans at $7 \square$ settings

44019

$8816\left[\mathrm{R}_{\text {int }}=0.0683\right]$

$1.592 \mathrm{~mm}^{-1}$

None

0.9538 and 0.5477 
Table 12 (cont.)

\section{Structure solution and Refinement}

Structure solution program

Primary solution method

Secondary solution method

Hydrogen placement

Structure refinement program

Refinement method

Data / restraints / parameters

Treatment of hydrogen atoms

Goodness-of-fit on $\mathrm{F}^{2}$

Final $R$ indices $[\mathrm{I}>2 \square(\mathrm{I}), 6545$ reflections]

$\mathrm{R}$ indices (all data)

Type of weighting scheme used

Weighting scheme used

Max shift/error

Average shift/error

Largest diff. peak and hole
SHELXS-97 (Sheldrick, 1990)

Direct methods

Difference Fourier map

Geometric positions

SHELXL-97 (Sheldrick, 1997)

Full matrix least-squares on $\mathrm{F}^{2}$

$8816 / 0 / 511$

Riding

1.731

$\mathrm{R} 1=0.0416, w \mathrm{R} 2=0.0744$

$\mathrm{R} 1=0.0657, w \mathrm{R} 2=0.0765$

Sigma

$w=1 / \square^{2}\left(\mathrm{Fo}^{2}\right)$

0.002

0.000

0.820 and -0.599 e. $\AA^{-3}$

\section{Special Refinement Details}

The crystals contain dichloromethane as a solvent of crystallization. There is one solvent site per asymmetric unit. The dichloromethane in this site is disordered between two orientations with relative occupancies of approximately 80:20 (see Table 2). The non-hydrogen atoms of both orientations were refined anisotropically. All hydrogen atoms in the structure were restrained to ride on the atoms to which they are bonded.

Refinement of $\mathrm{F}^{2}$ against ALL reflections. The weighted R-factor $(w \mathrm{R})$ and goodness of fit $(\mathrm{S})$ are based on $\mathrm{F}^{2}$, conventional $\mathrm{R}$-factors $(\mathrm{R})$ are based on $\mathrm{F}$, with $\mathrm{F}$ set to zero for negative $\mathrm{F}^{2}$. The threshold expression of $\mathrm{F}^{2}>$ $2 \square\left(\mathrm{F}^{2}\right)$ is used only for calculating $\mathrm{R}$-factors $(\mathrm{gt})$ etc. and is not relevant to the choice of reflections for refinement. $\mathrm{R}$-factors based on $\mathrm{F}^{2}$ are statistically about twice as large as those based on $\mathrm{F}$, and R-factors based on ALL data will be even larger.

All esds (except the esd in the dihedral angle between two 1.s. planes) are estimated using the full covariance matrix. The cell esds are taken into account individually in the estimation of esds in distances, angles and torsion angles; correlations between esds in cell parameters are only used when they are defined by crystal symmetry. An approximate (isotropic) treatment of cell esds is used for estimating esds involving l.s. planes. 
Table 13. Atomic coordinates $\left(x 1^{4}\right)$ and equivalent isotropic displacement parameters $\left(\AA^{2} \times 1^{3}\right)$ for $4(C C D C 208405)$. $U(e q)$ is defined as the trace of the orthogonalized $U^{i j}$ tensor.

\begin{tabular}{|c|c|c|c|c|c|}
\hline & $\mathrm{X}$ & $\mathrm{y}$ & $\mathrm{z}$ & $\mathrm{U}_{\mathrm{eq}}$ & Occ \\
\hline $\operatorname{Cr}(1)$ & $8942(1)$ & $8259(1)$ & $1854(1)$ & $15(1)$ & 1 \\
\hline $\operatorname{Br}(1)$ & $9932(1)$ & $9902(1)$ & $1865(1)$ & $22(1)$ & 1 \\
\hline $\mathrm{P}(1)$ & $7332(1)$ & $7132(1)$ & $1821(1)$ & $15(1)$ & 1 \\
\hline $\mathrm{P}(2)$ & $6368(1)$ & $8830(1)$ & $2778(1)$ & $15(1)$ & 1 \\
\hline $\mathrm{O}(1)$ & $7653(2)$ & $9266(2)$ & $850(1)$ & $15(1)$ & 1 \\
\hline $\mathrm{O}(2)$ & $9422(2)$ & $5153(2)$ & $1140(1)$ & $22(1)$ & 1 \\
\hline $\mathrm{O}(3)$ & $7145(2)$ & $9783(2)$ & $3856(1)$ & $24(1)$ & 1 \\
\hline $\mathrm{O}(4)$ & $3154(2)$ & $9653(2)$ & $3397(1)$ & $26(1)$ & 1 \\
\hline $\mathrm{N}(1)$ & $5833(2)$ & $7901(2)$ & $2386(1)$ & $16(1)$ & 1 \\
\hline $\mathrm{C}(1)$ & $6911(3)$ & $7580(2)$ & $854(2)$ & $14(1)$ & 1 \\
\hline $\mathrm{C}(2)$ & $6385(3)$ & $6943(3)$ & $490(2)$ & $18(1)$ & 1 \\
\hline $\mathrm{C}(3)$ & $6068(3)$ & $7381(3)$ & $-250(2)$ & $19(1)$ & 1 \\
\hline $\mathrm{C}(4)$ & $6306(3)$ & $8452(3)$ & $-631(2)$ & $20(1)$ & 1 \\
\hline $\mathrm{C}(5)$ & $6826(3)$ & $9103(2)$ & $-290(2)$ & $17(1)$ & 1 \\
\hline$C(6)$ & $7123(3)$ & $8668(2)$ & $455(2)$ & $13(1)$ & 1 \\
\hline $\mathrm{C}(7)$ & $7850(3)$ & $10396(2)$ & $434(2)$ & $19(1)$ & 1 \\
\hline $\mathrm{C}(8)$ & $7384(3)$ & $5576(2)$ & 2101(2) & $18(1)$ & 1 \\
\hline $\mathrm{C}(9)$ & $6374(3)$ & $5205(3)$ & $2700(2)$ & $21(1)$ & 1 \\
\hline$C(10)$ & $6494(3)$ & $3998(3)$ & $2933(2)$ & $25(1)$ & 1 \\
\hline $\mathrm{C}(11)$ & $7665(3)$ & $3148(3)$ & $2569(2)$ & $25(1)$ & 1 \\
\hline $\mathrm{C}(12)$ & $8681(3)$ & $3484(3)$ & 1981(2) & $22(1)$ & 1 \\
\hline $\mathrm{C}(13)$ & $8533(3)$ & $4705(3)$ & 1734(2) & $19(1)$ & 1 \\
\hline$C(14)$ & 10711(3) & $4314(3)$ & $799(2)$ & $26(1)$ & 1 \\
\hline$C(15)$ & $5986(3)$ & $8402(2)$ & $3830(2)$ & $19(1)$ & 1 \\
\hline$C(16)$ & $5264(4)$ & $7569(3)$ & $4204(2)$ & $33(1)$ & 1 \\
\hline$C(17)$ & $5071(4)$ & $7266(3)$ & $5004(2)$ & $40(1)$ & 1 \\
\hline $\mathrm{C}(18)$ & $5647(4)$ & $7776(3)$ & $5432(2)$ & $37(1)$ & 1 \\
\hline C(19) & $6353(3)$ & $8624(3)$ & $5073(2)$ & $24(1)$ & 1 \\
\hline $\mathrm{C}(20)$ & $6495(3)$ & $8946(2)$ & $4277(2)$ & $18(1)$ & 1 \\
\hline $\mathrm{C}(21)$ & $7961(4)$ & $10183(3)$ & $4244(2)$ & $37(1)$ & 1 \\
\hline $\mathrm{C}(22)$ & $5096(3)$ & $10322(2)$ & $2641(2)$ & $16(1)$ & 1 \\
\hline $\mathrm{C}(23)$ & $5645(3)$ & $11252(2)$ & $2229(2)$ & $17(1)$ & 1 \\
\hline $\mathrm{C}(24)$ & $4742(3)$ & $12418(3)$ & $2124(2)$ & $23(1)$ & 1 \\
\hline $\mathrm{C}(25)$ & $3270(3)$ & $12647(3)$ & $2424(2)$ & $26(1)$ & 1 \\
\hline $\mathrm{C}(26)$ & $2700(3)$ & $11749(3)$ & $2831(2)$ & $24(1)$ & 1 \\
\hline $\mathrm{C}(27)$ & $3600(3)$ & $10585(3)$ & $2960(2)$ & $19(1)$ & 1 \\
\hline $\mathrm{C}(28)$ & $1655(3)$ & $9780(3)$ & $3634(2)$ & $30(1)$ & 1 \\
\hline C(29) & $4339(3)$ & $8003(3)$ & 2291(2) & $21(1)$ & 1 \\
\hline $\mathrm{C}(30)$ & $10162(3)$ & $7095(3)$ & $2620(2)$ & $20(1)$ & 1 \\
\hline $\mathrm{C}(31)$ & 9896(3) & $6997(3)$ & $3422(2)$ & $29(1)$ & 1 \\
\hline $\mathrm{C}(32)$ & 10899(3) & $6254(3)$ & $3873(2)$ & $33(1)$ & 1 \\
\hline $\mathrm{C}(33)$ & $12237(3)$ & $5578(3)$ & $3510(2)$ & $29(1)$ & 1 \\
\hline $\mathrm{C}(34)$ & $12535(3)$ & $5650(3)$ & $2721(2)$ & $24(1)$ & 1 \\
\hline $\mathrm{C}(35)$ & $11526(3)$ & $6391(2)$ & $2261(2)$ & $18(1)$ & 1 \\
\hline$C(36)$ & $11764(3)$ & $6540(2)$ & 1413(2) & $19(1)$ & 1 \\
\hline $\mathrm{C}(37)$ & $13018(3)$ & $5914(3)$ & $959(2)$ & $22(1)$ & 1 \\
\hline $\mathrm{C}(38)$ & $13125(3)$ & $6110(3)$ & $151(2)$ & $26(1)$ & 1 \\
\hline
\end{tabular}




\begin{tabular}{lclrrl}
$\mathrm{C}(39)$ & $12033(3)$ & $6886(3)$ & $-195(2)$ & $22(1)$ & 1 \\
$\mathrm{C}(40)$ & $10761(3)$ & $7525(2)$ & $268(2)$ & $20(1)$ & 1 \\
$\mathrm{C}(41)$ & $10607(3)$ & $7379(2)$ & $1067(2)$ & $15(1)$ & 1 \\
& & & & & \\
$\mathrm{C}(51)$ & $9223(11)$ & $4067(8)$ & $3880(6)$ & $32(2)$ & $0.808(2)$ \\
$\mathrm{Cl}(1)$ & $7689(1)$ & $5096(1)$ & $4350(1)$ & $34(1)$ & $0.808(2)$ \\
$\mathrm{Cl}(2)$ & $9251(1)$ & $2575(1)$ & $4336(1)$ & $36(1)$ & $0.808(2)$ \\
& & & & & \\
$\mathrm{C}(51 \mathrm{~B})$ & $8800(50)$ & $4220(60)$ & $4050(30)$ & $90(20)$ & $0.192(2)$ \\
$\mathrm{Cl}(1 \mathrm{~B})$ & $7334(11)$ & $3765(8)$ & $4655(5)$ & $123(4)$ & $0.192(2)$ \\
$\mathrm{Cl}(2 \mathrm{~B})$ & $10471(12)$ & $3352(9)$ & $4230(6)$ & $137(4)$ & $0.192(2)$ \\
& & & & & \\
\hline
\end{tabular}

Table 14. Selected bond lengths $[\AA]$ and angles $\left[^{\circ}\right]$ for 4 (CCDC 208405).

\begin{tabular}{lllr}
\hline $\mathrm{Cr}(1)-\mathrm{C}(30)$ & $2.035(3)$ & $\mathrm{C}(30)-\operatorname{Cr}(1)-\mathrm{C}(41)$ & $81.95(11)$ \\
$\mathrm{Cr}(1)-\mathrm{C}(41)$ & $2.058(3)$ & $\mathrm{C}(30)-\operatorname{Cr}(1)-\mathrm{O}(1)$ & $168.61(9)$ \\
$\mathrm{Cr}(1)-\mathrm{O}(1)$ & $2.337(2)$ & $\mathrm{C}(41)-\operatorname{Cr}(1)-\mathrm{O}(1)$ & $89.56(9)$ \\
$\mathrm{Cr}(1)-\mathrm{P}(1)$ & $2.4261(8)$ & $\mathrm{C}(30)-\operatorname{Cr}(1)-\mathrm{P}(1)$ & $100.01(8)$ \\
$\mathrm{Cr}(1)-\mathrm{Br}(1)$ & $2.4815(5)$ & $\mathrm{C}(41)-\operatorname{Cr}(1)-\mathrm{P}(1)$ & $94.21(7)$ \\
$\mathrm{Cr}(1)-\mathrm{P}(2)$ & $2.6608(8)$ & $\mathrm{O}(1)-\operatorname{Cr}(1)-\mathrm{P}(1)$ & $72.94(5)$ \\
& & $\mathrm{C}(30)-\operatorname{Cr}(1)-\operatorname{Br}(1)$ & $93.24(8)$ \\
& & $\mathrm{C}(41)-\operatorname{Cr}(1)-\operatorname{Br}(1)$ & $97.74(7)$ \\
& $\mathrm{O}(1)-\operatorname{Cr}(1)-\operatorname{Br}(1)$ & $95.43(5)$ \\
& $\mathrm{P}(1)-\operatorname{Cr}(1)-\operatorname{Br}(1)$ & $163.28(3)$ \\
& $\mathrm{C}(30)-\operatorname{Cr}(1)-\mathrm{P}(2)$ & $100.21(8)$ \\
& $\mathrm{C}(41)-\operatorname{Cr}(1)-\mathrm{P}(2)$ & $159.08(8)$ \\
& & $\mathrm{O}(1)-\operatorname{Cr}(1)-\mathrm{P}(2)$ & $85.06(5)$ \\
& $\mathrm{P}(1)-\operatorname{Cr}(1)-\mathrm{P}(2)$ & $64.89(2)$ \\
& $\mathrm{Br}(1)-\operatorname{Cr}(1)-\mathrm{P}(2)$ & $102.88(2)$ \\
\hline
\end{tabular}


Table 15. Bond lengths $[\AA ̊ \AA]$ and angles $\left[{ }^{\circ}\right]$ for 4 (CCDC 208405).

\begin{tabular}{|c|c|c|c|}
\hline $\mathrm{Cr}(1)-\mathrm{C}(30)$ & $2.035(3)$ & $\mathrm{C}(17)-\mathrm{C}(18)$ & $1.392(4)$ \\
\hline $\mathrm{Cr}(1)-\mathrm{C}(41)$ & $2.058(3)$ & $\mathrm{C}(17)-\mathrm{H}(17)$ & 0.9500 \\
\hline $\mathrm{Cr}(1)-\mathrm{O}(1)$ & $2.3369(19)$ & $\mathrm{C}(18)-\mathrm{C}(19)$ & $1.383(4)$ \\
\hline $\mathrm{Cr}(1)-\mathrm{P}(1)$ & $2.4261(8)$ & $\mathrm{C}(18)-\mathrm{H}(18)$ & 0.9500 \\
\hline $\operatorname{Cr}(1)-\operatorname{Br}(1)$ & $2.4815(5)$ & $\mathrm{C}(19)-\mathrm{C}(20)$ & $1.385(4)$ \\
\hline $\mathrm{Cr}(1)-\mathrm{P}(2)$ & $2.6608(8)$ & $\mathrm{C}(19)-\mathrm{H}(19)$ & 0.9500 \\
\hline $\mathrm{P}(1)-\mathrm{N}(1)$ & $1.697(2)$ & $\mathrm{C}(21)-\mathrm{H}(21 \mathrm{~A})$ & 0.9800 \\
\hline $\mathrm{P}(1)-\mathrm{C}(1)$ & $1.808(3)$ & $\mathrm{C}(21)-\mathrm{H}(21 \mathrm{~B})$ & 0.9800 \\
\hline $\mathrm{P}(1)-\mathrm{C}(8)$ & $1.810(3)$ & $\mathrm{C}(21)-\mathrm{H}(21 \mathrm{C})$ & 0.9800 \\
\hline $\mathrm{P}(2)-\mathrm{N}(1)$ & $1.712(2)$ & $\mathrm{C}(22)-\mathrm{C}(23)$ & $1.394(4)$ \\
\hline $\mathrm{P}(2)-\mathrm{C}(22)$ & $1.820(3)$ & $C(22)-C(27)$ & $1.413(4)$ \\
\hline $\mathrm{P}(2)-\mathrm{C}(15)$ & $1.829(3)$ & $C(23)-C(24)$ & $1.388(4)$ \\
\hline $\mathrm{O}(1)-\mathrm{C}(6)$ & $1.394(3)$ & $\mathrm{C}(23)-\mathrm{H}(23)$ & 0.9500 \\
\hline $\mathrm{O}(1)-\mathrm{C}(7)$ & $1.448(3)$ & $C(24)-C(25)$ & $1.388(4)$ \\
\hline $\mathrm{O}(2)-\mathrm{C}(13)$ & $1.350(3)$ & $\mathrm{C}(24)-\mathrm{H}(24)$ & 0.9500 \\
\hline $\mathrm{O}(2)-\mathrm{C}(14)$ & $1.439(3)$ & $C(25)-C(26)$ & $1.370(4)$ \\
\hline $\mathrm{O}(3)-\mathrm{C}(20)$ & $1.363(3)$ & $\mathrm{C}(25)-\mathrm{H}(25)$ & 0.9500 \\
\hline $\mathrm{O}(3)-\mathrm{C}(21)$ & $1.430(3)$ & $C(26)-C(27)$ & $1.389(4)$ \\
\hline $\mathrm{O}(4)-\mathrm{C}(27)$ & $1.357(3)$ & $\mathrm{C}(26)-\mathrm{H}(26)$ & 0.9500 \\
\hline $\mathrm{O}(4)-\mathrm{C}(28)$ & $1.412(3)$ & $\mathrm{C}(28)-\mathrm{H}(28 \mathrm{~A})$ & 0.9800 \\
\hline $\mathrm{N}(1)-\mathrm{C}(29)$ & $1.493(3)$ & $\mathrm{C}(28)-\mathrm{H}(28 \mathrm{~B})$ & 0.9800 \\
\hline C(1)-C(2) & $1.388(4)$ & $\mathrm{C}(28)-\mathrm{H}(28 \mathrm{C})$ & 0.9800 \\
\hline$C(1)-C(6)$ & $1.400(4)$ & $\mathrm{C}(29)-\mathrm{H}(29 \mathrm{~A})$ & 0.9800 \\
\hline$C(2)-C(3)$ & $1.388(4)$ & C(29)-H(29B) & 0.9800 \\
\hline $\mathrm{C}(2)-\mathrm{H}(2)$ & 0.9500 & C(29)-H(29C) & 0.9800 \\
\hline C(3)-C(4) & $1.384(4)$ & C(30)-C(31) & $1.387(4)$ \\
\hline C(3)-H(3) & 0.9500 & C(30)-C(35) & $1.425(4)$ \\
\hline$C(4)-C(5)$ & $1.371(4)$ & C(31)-C(32) & $1.385(4)$ \\
\hline C(4)-H(4) & 0.9500 & $\mathrm{C}(31)-\mathrm{H}(31)$ & 0.9500 \\
\hline$C(5)-C(6)$ & $1.390(4)$ & C(32)-C(33) & $1.397(4)$ \\
\hline C(5)-H(5) & 0.9500 & $\mathrm{C}(32)-\mathrm{H}(32)$ & 0.9500 \\
\hline $\mathrm{C}(7)-\mathrm{H}(7 \mathrm{~A})$ & 0.9800 & C(33)-C(34) & $1.369(4)$ \\
\hline $\mathrm{C}(7)-\mathrm{H}(7 \mathrm{~B})$ & 0.9800 & C(33)-H(33) & 0.9500 \\
\hline $\mathrm{C}(7)-\mathrm{H}(7 \mathrm{C})$ & 0.9800 & C(34)-C(35) & $1.398(4)$ \\
\hline C(8)-C(9) & $1.392(4)$ & $\mathrm{C}(34)-\mathrm{H}(34)$ & 0.9500 \\
\hline$C(8)-C(13)$ & $1.408(4)$ & C(35)-C(36) & $1.464(4)$ \\
\hline$C(9)-C(10)$ & $1.389(4)$ & $C(36)-C(37)$ & $1.396(4)$ \\
\hline $\mathrm{C}(9)-\mathrm{H}(9)$ & 0.9500 & $C(36)-C(41)$ & $1.419(4)$ \\
\hline $\mathrm{C}(10)-\mathrm{C}(11)$ & $1.401(4)$ & C(37)-C(38) & $1.402(4)$ \\
\hline $\mathrm{C}(10)-\mathrm{H}(10)$ & 0.9500 & $\mathrm{C}(37)-\mathrm{H}(37)$ & 0.9500 \\
\hline C(11)-C(12) & $1.368(4)$ & C(38)-C(39) & $1.351(4)$ \\
\hline $\mathrm{C}(11)-\mathrm{H}(11)$ & 0.9500 & $\mathrm{C}(38)-\mathrm{H}(38)$ & 0.9500 \\
\hline C(12)-C(13) & $1.403(4)$ & C(39)-C(40) & $1.418(4)$ \\
\hline $\mathrm{C}(12)-\mathrm{H}(12)$ & 0.9500 & C(39)-H(39) & 0.9500 \\
\hline $\mathrm{C}(14)-\mathrm{H}(14 \mathrm{~A})$ & 0.9800 & $\mathrm{C}(40)-\mathrm{C}(41)$ & $1.387(4)$ \\
\hline $\mathrm{C}(14)-\mathrm{H}(14 \mathrm{~B})$ & 0.9800 & $\mathrm{C}(40)-\mathrm{H}(40)$ & 0.9500 \\
\hline $\mathrm{C}(14)-\mathrm{H}(14 \mathrm{C})$ & 0.9800 & $\mathrm{C}(51)-\mathrm{Cl}(1)$ & $1.777(9)$ \\
\hline C(15)-C(16) & $1.384(4)$ & $\mathrm{C}(51)-\mathrm{Cl}(2)$ & $1.793(10)$ \\
\hline C(15)-C(20) & $1.401(4)$ & $\mathrm{C}(51)-\mathrm{H}(51 \mathrm{~A})$ & 0.9900 \\
\hline $\mathrm{C}(16)-\mathrm{C}(17)$ & $1.386(4)$ & C(51)-H(51B) & 0.9900 \\
\hline $\mathrm{C}(16)-\mathrm{H}(16)$ & 0.9500 & $\mathrm{C}(51 \mathrm{~B})-\mathrm{Cl}(2 \mathrm{~B})$ & $1.70(5)$ \\
\hline
\end{tabular}




\begin{tabular}{|c|c|c|c|}
\hline $\mathrm{C}(51 \mathrm{~B})-\mathrm{Cl}(1 \mathrm{~B})$ & $1.77(5)$ & $\mathrm{C}(6)-\mathrm{C}(5)-\mathrm{H}(5)$ & 120.6 \\
\hline $\mathrm{C}(51 \mathrm{~B})-\mathrm{H}(51 \mathrm{C})$ & 0.9900 & $\mathrm{C}(5)-\mathrm{C}(6)-\mathrm{O}(1)$ & $123.0(2)$ \\
\hline \multirow[t]{2}{*}{$\mathrm{C}(51 \mathrm{~B})-\mathrm{H}(51 \mathrm{D})$} & 0.9900 & $C(5)-C(6)-C(1)$ & $121.1(2)$ \\
\hline & & $\mathrm{O}(1)-\mathrm{C}(6)-\mathrm{C}(1)$ & $115.9(2)$ \\
\hline $\mathrm{C}(30)-\mathrm{Cr}(1)-\mathrm{C}(41)$ & $81.95(11)$ & $\mathrm{O}(1)-\mathrm{C}(7)-\mathrm{H}(7 \mathrm{~A})$ & 109.5 \\
\hline $\mathrm{C}(30)-\mathrm{Cr}(1)-\mathrm{O}(1)$ & $168.61(9)$ & $\mathrm{O}(1)-\mathrm{C}(7)-\mathrm{H}(7 \mathrm{~B})$ & 109.5 \\
\hline $\mathrm{C}(41)-\mathrm{Cr}(1)-\mathrm{O}(1)$ & $89.56(9)$ & $\mathrm{H}(7 \mathrm{~A})-\mathrm{C}(7)-\mathrm{H}(7 \mathrm{~B})$ & 109.5 \\
\hline $\mathrm{C}(30)-\mathrm{Cr}(1)-\mathrm{P}(1)$ & $100.01(8)$ & $\mathrm{O}(1)-\mathrm{C}(7)-\mathrm{H}(7 \mathrm{C})$ & 109.5 \\
\hline $\mathrm{C}(41)-\mathrm{Cr}(1)-\mathrm{P}(1)$ & $94.21(7)$ & $\mathrm{H}(7 \mathrm{~A})-\mathrm{C}(7)-\mathrm{H}(7 \mathrm{C})$ & 109.5 \\
\hline $\mathrm{O}(1)-\mathrm{Cr}(1)-\mathrm{P}(1)$ & $72.94(5)$ & $\mathrm{H}(7 \mathrm{~B})-\mathrm{C}(7)-\mathrm{H}(7 \mathrm{C})$ & 109.5 \\
\hline $\mathrm{C}(30)-\mathrm{Cr}(1)-\mathrm{Br}(1)$ & $93.24(8)$ & $\mathrm{C}(9)-\mathrm{C}(8)-\mathrm{C}(13)$ & $118.8(3)$ \\
\hline $\mathrm{C}(41)-\mathrm{Cr}(1)-\mathrm{Br}(1)$ & $97.74(7)$ & $\mathrm{C}(9)-\mathrm{C}(8)-\mathrm{P}(1)$ & $122.0(2)$ \\
\hline $\mathrm{O}(1)-\mathrm{Cr}(1)-\mathrm{Br}(1)$ & $95.43(5)$ & $\mathrm{C}(13)-\mathrm{C}(8)-\mathrm{P}(1)$ & $119.0(2)$ \\
\hline $\mathrm{P}(1)-\mathrm{Cr}(1)-\mathrm{Br}(1)$ & $163.28(3)$ & $\mathrm{C}(10)-\mathrm{C}(9)-\mathrm{C}(8)$ & $120.7(3)$ \\
\hline $\mathrm{C}(30)-\mathrm{Cr}(1)-\mathrm{P}(2)$ & $100.21(8)$ & $\mathrm{C}(10)-\mathrm{C}(9)-\mathrm{H}(9)$ & 119.6 \\
\hline $\mathrm{C}(41)-\mathrm{Cr}(1)-\mathrm{P}(2)$ & $159.08(8)$ & $\mathrm{C}(8)-\mathrm{C}(9)-\mathrm{H}(9)$ & 119.6 \\
\hline $\mathrm{O}(1)-\mathrm{Cr}(1)-\mathrm{P}(2)$ & $85.06(5)$ & $C(9)-C(10)-C(11)$ & $119.3(3)$ \\
\hline $\mathrm{P}(1)-\mathrm{Cr}(1)-\mathrm{P}(2)$ & $64.89(2)$ & C(9)-C(10)-H(10) & 120.3 \\
\hline $\operatorname{Br}(1)-\mathrm{Cr}(1)-\mathrm{P}(2)$ & $102.88(2)$ & $\mathrm{C}(11)-\mathrm{C}(10)-\mathrm{H}(10)$ & 120.3 \\
\hline $\mathrm{N}(1)-\mathrm{P}(1)-\mathrm{C}(1)$ & $104.37(12)$ & $\mathrm{C}(12)-\mathrm{C}(11)-\mathrm{C}(10)$ & $121.4(3)$ \\
\hline $\mathrm{N}(1)-\mathrm{P}(1)-\mathrm{C}(8)$ & $105.67(12)$ & $\mathrm{C}(12)-\mathrm{C}(11)-\mathrm{H}(11)$ & 119.3 \\
\hline $\mathrm{C}(1)-\mathrm{P}(1)-\mathrm{C}(8)$ & $106.58(13)$ & $\mathrm{C}(10)-\mathrm{C}(11)-\mathrm{H}(11)$ & 119.3 \\
\hline $\mathrm{N}(1)-\mathrm{P}(1)-\mathrm{Cr}(1)$ & $97.96(8)$ & $\mathrm{C}(11)-\mathrm{C}(12)-\mathrm{C}(13)$ & $119.1(3)$ \\
\hline $\mathrm{C}(1)-\mathrm{P}(1)-\mathrm{Cr}(1)$ & 104.71(9) & $\mathrm{C}(11)-\mathrm{C}(12)-\mathrm{H}(12)$ & 120.4 \\
\hline $\mathrm{C}(8)-\mathrm{P}(1)-\mathrm{Cr}(1)$ & $134.14(9)$ & $\mathrm{C}(13)-\mathrm{C}(12)-\mathrm{H}(12)$ & 120.4 \\
\hline $\mathrm{N}(1)-\mathrm{P}(2)-\mathrm{C}(22)$ & $108.23(12)$ & $\mathrm{O}(2)-\mathrm{C}(13)-\mathrm{C}(12)$ & $124.9(3)$ \\
\hline $\mathrm{N}(1)-\mathrm{P}(2)-\mathrm{C}(15)$ & $106.44(12)$ & $\mathrm{O}(2)-\mathrm{C}(13)-\mathrm{C}(8)$ & $114.4(2)$ \\
\hline $\mathrm{C}(22)-\mathrm{P}(2)-\mathrm{C}(15)$ & $101.96(12)$ & $\mathrm{C}(12)-\mathrm{C}(13)-\mathrm{C}(8)$ & $120.6(3)$ \\
\hline N(1)-P(2)-Cr(1) & 89.33(8) & $\mathrm{O}(2)-\mathrm{C}(14)-\mathrm{H}(14 \mathrm{~A})$ & 109.5 \\
\hline $\mathrm{C}(22)-\mathrm{P}(2)-\mathrm{Cr}(1)$ & $121.14(9)$ & $\mathrm{O}(2)-\mathrm{C}(14)-\mathrm{H}(14 \mathrm{~B})$ & 109.5 \\
\hline $\mathrm{C}(15)-\mathrm{P}(2)-\mathrm{Cr}(1)$ & $126.73(9)$ & $\mathrm{H}(14 \mathrm{~A})-\mathrm{C}(14)-\mathrm{H}(14 \mathrm{~B})$ & 109.5 \\
\hline $\mathrm{C}(6)-\mathrm{O}(1)-\mathrm{C}(7)$ & $115.2(2)$ & $\mathrm{O}(2)-\mathrm{C}(14)-\mathrm{H}(14 \mathrm{C})$ & 109.5 \\
\hline $\mathrm{C}(6)-\mathrm{O}(1)-\mathrm{Cr}(1)$ & $121.70(15)$ & $\mathrm{H}(14 \mathrm{~A})-\mathrm{C}(14)-\mathrm{H}(14 \mathrm{C})$ & 109.5 \\
\hline $\mathrm{C}(7)-\mathrm{O}(1)-\mathrm{Cr}(1)$ & $119.23(15)$ & $\mathrm{H}(14 \mathrm{~B})-\mathrm{C}(14)-\mathrm{H}(14 \mathrm{C})$ & 109.5 \\
\hline $\mathrm{C}(13)-\mathrm{O}(2)-\mathrm{C}(14)$ & $117.1(2)$ & $C(16)-C(15)-C(20)$ & $118.7(3)$ \\
\hline $\mathrm{C}(20)-\mathrm{O}(3)-\mathrm{C}(21)$ & $116.8(2)$ & $\mathrm{C}(16)-\mathrm{C}(15)-\mathrm{P}(2)$ & $124.5(2)$ \\
\hline $\mathrm{C}(27)-\mathrm{O}(4)-\mathrm{C}(28)$ & $119.8(2)$ & $\mathrm{C}(20)-\mathrm{C}(15)-\mathrm{P}(2)$ & $116.8(2)$ \\
\hline $\mathrm{C}(29)-\mathrm{N}(1)-\mathrm{P}(1)$ & $121.55(18)$ & $\mathrm{C}(15)-\mathrm{C}(16)-\mathrm{C}(17)$ & $120.5(3)$ \\
\hline $\mathrm{C}(29)-\mathrm{N}(1)-\mathrm{P}(2)$ & $128.34(18)$ & $\mathrm{C}(15)-\mathrm{C}(16)-\mathrm{H}(16)$ & 119.7 \\
\hline $\mathrm{P}(1)-\mathrm{N}(1)-\mathrm{P}(2)$ & $106.76(12)$ & $\mathrm{C}(17)-\mathrm{C}(16)-\mathrm{H}(16)$ & 119.7 \\
\hline$C(2)-C(1)-C(6)$ & $118.5(3)$ & $\mathrm{C}(16)-\mathrm{C}(17)-\mathrm{C}(18)$ & $119.8(3)$ \\
\hline $\mathrm{C}(2)-\mathrm{C}(1)-\mathrm{P}(1)$ & $125.3(2)$ & $\mathrm{C}(16)-\mathrm{C}(17)-\mathrm{H}(17)$ & 120.1 \\
\hline $\mathrm{C}(6)-\mathrm{C}(1)-\mathrm{P}(1)$ & $116.2(2)$ & $\mathrm{C}(18)-\mathrm{C}(17)-\mathrm{H}(17)$ & 120.1 \\
\hline$C(3)-C(2)-C(1)$ & $120.6(3)$ & $\mathrm{C}(19)-\mathrm{C}(18)-\mathrm{C}(17)$ & $120.8(3)$ \\
\hline $\mathrm{C}(3)-\mathrm{C}(2)-\mathrm{H}(2)$ & 119.7 & $\mathrm{C}(19)-\mathrm{C}(18)-\mathrm{H}(18)$ & 119.6 \\
\hline $\mathrm{C}(1)-\mathrm{C}(2)-\mathrm{H}(2)$ & 119.7 & $\mathrm{C}(17)-\mathrm{C}(18)-\mathrm{H}(18)$ & 119.6 \\
\hline$C(4)-C(3)-C(2)$ & $119.4(3)$ & $\mathrm{C}(18)-\mathrm{C}(19)-\mathrm{C}(20)$ & $118.8(3)$ \\
\hline $\mathrm{C}(4)-\mathrm{C}(3)-\mathrm{H}(3)$ & 120.3 & $\mathrm{C}(18)-\mathrm{C}(19)-\mathrm{H}(19)$ & 120.6 \\
\hline $\mathrm{C}(2)-\mathrm{C}(3)-\mathrm{H}(3)$ & 120.3 & $\mathrm{C}(20)-\mathrm{C}(19)-\mathrm{H}(19)$ & 120.6 \\
\hline$C(5)-C(4)-C(3)$ & $121.5(3)$ & $\mathrm{O}(3)-\mathrm{C}(20)-\mathrm{C}(19)$ & $124.5(3)$ \\
\hline $\mathrm{C}(5)-\mathrm{C}(4)-\mathrm{H}(4)$ & 119.2 & $\mathrm{O}(3)-\mathrm{C}(20)-\mathrm{C}(15)$ & $114.1(2)$ \\
\hline $\mathrm{C}(3)-\mathrm{C}(4)-\mathrm{H}(4)$ & 119.2 & $C(19)-C(20)-C(15)$ & $121.4(3)$ \\
\hline$C(4)-C(5)-C(6)$ & $118.8(3)$ & $\mathrm{O}(3)-\mathrm{C}(21)-\mathrm{H}(21 \mathrm{~A})$ & 109.5 \\
\hline $\mathrm{C}(4)-\mathrm{C}(5)-\mathrm{H}(5)$ & 120.6 & $\mathrm{O}(3)-\mathrm{C}(21)-\mathrm{H}(21 \mathrm{~B})$ & 109.5 \\
\hline
\end{tabular}




\begin{tabular}{|c|c|c|c|}
\hline $\mathrm{H}(21 \mathrm{~A})-\mathrm{C}(21)-\mathrm{H}(21 \mathrm{~B})$ & 109.5 & $\mathrm{C}(31)-\mathrm{C}(32)-\mathrm{H}(32)$ & 120.5 \\
\hline $\mathrm{O}(3)-\mathrm{C}(21)-\mathrm{H}(21 \mathrm{C})$ & 109.5 & $\mathrm{C}(33)-\mathrm{C}(32)-\mathrm{H}(32)$ & 120.5 \\
\hline $\mathrm{H}(21 \mathrm{~A})-\mathrm{C}(21)-\mathrm{H}(21 \mathrm{C})$ & 109.5 & $\mathrm{C}(34)-\mathrm{C}(33)-\mathrm{C}(32)$ & $120.0(3)$ \\
\hline $\mathrm{H}(21 \mathrm{~B})-\mathrm{C}(21)-\mathrm{H}(21 \mathrm{C})$ & 109.5 & $\mathrm{C}(34)-\mathrm{C}(33)-\mathrm{H}(33)$ & 120.0 \\
\hline $\mathrm{C}(23)-\mathrm{C}(22)-\mathrm{C}(27)$ & $118.8(3)$ & $\mathrm{C}(32)-\mathrm{C}(33)-\mathrm{H}(33)$ & 120.0 \\
\hline $\mathrm{C}(23)-\mathrm{C}(22)-\mathrm{P}(2)$ & $118.0(2)$ & $\mathrm{C}(33)-\mathrm{C}(34)-\mathrm{C}(35)$ & $121.4(3)$ \\
\hline $\mathrm{C}(27)-\mathrm{C}(22)-\mathrm{P}(2)$ & $123.2(2)$ & $\mathrm{C}(33)-\mathrm{C}(34)-\mathrm{H}(34)$ & 119.3 \\
\hline $\mathrm{C}(24)-\mathrm{C}(23)-\mathrm{C}(22)$ & $120.9(3)$ & $\mathrm{C}(35)-\mathrm{C}(34)-\mathrm{H}(34)$ & 119.3 \\
\hline $\mathrm{C}(24)-\mathrm{C}(23)-\mathrm{H}(23)$ & 119.5 & $\mathrm{C}(34)-\mathrm{C}(35)-\mathrm{C}(30)$ & $119.3(3)$ \\
\hline $\mathrm{C}(22)-\mathrm{C}(23)-\mathrm{H}(23)$ & 119.5 & $\mathrm{C}(34)-\mathrm{C}(35)-\mathrm{C}(36)$ & $125.1(3)$ \\
\hline $\mathrm{C}(23)-\mathrm{C}(24)-\mathrm{C}(25)$ & 119.1(3) & $\mathrm{C}(30)-\mathrm{C}(35)-\mathrm{C}(36)$ & $115.5(2)$ \\
\hline $\mathrm{C}(23)-\mathrm{C}(24)-\mathrm{H}(24)$ & 120.4 & $\mathrm{C}(37)-\mathrm{C}(36)-\mathrm{C}(41)$ & $120.8(3)$ \\
\hline $\mathrm{C}(25)-\mathrm{C}(24)-\mathrm{H}(24)$ & 120.4 & $\mathrm{C}(37)-\mathrm{C}(36)-\mathrm{C}(35)$ & $124.0(3)$ \\
\hline $\mathrm{C}(26)-\mathrm{C}(25)-\mathrm{C}(24)$ & $121.2(3)$ & $\mathrm{C}(41)-\mathrm{C}(36)-\mathrm{C}(35)$ & $115.2(2)$ \\
\hline $\mathrm{C}(26)-\mathrm{C}(25)-\mathrm{H}(25)$ & 119.4 & $\mathrm{C}(36)-\mathrm{C}(37)-\mathrm{C}(38)$ & $119.4(3)$ \\
\hline $\mathrm{C}(24)-\mathrm{C}(25)-\mathrm{H}(25)$ & 119.4 & $\mathrm{C}(36)-\mathrm{C}(37)-\mathrm{H}(37)$ & 120.3 \\
\hline $\mathrm{C}(25)-\mathrm{C}(26)-\mathrm{C}(27)$ & $120.2(3)$ & $\mathrm{C}(38)-\mathrm{C}(37)-\mathrm{H}(37)$ & 120.3 \\
\hline $\mathrm{C}(25)-\mathrm{C}(26)-\mathrm{H}(26)$ & 119.9 & C(39)-C(38)-C(37) & $121.2(3)$ \\
\hline $\mathrm{C}(27)-\mathrm{C}(26)-\mathrm{H}(26)$ & 119.9 & $\mathrm{C}(39)-\mathrm{C}(38)-\mathrm{H}(38)$ & 119.4 \\
\hline $\mathrm{O}(4)-\mathrm{C}(27)-\mathrm{C}(26)$ & $124.5(3)$ & $\mathrm{C}(37)-\mathrm{C}(38)-\mathrm{H}(38)$ & 119.4 \\
\hline $\mathrm{O}(4)-\mathrm{C}(27)-\mathrm{C}(22)$ & $115.8(2)$ & $\mathrm{C}(38)-\mathrm{C}(39)-\mathrm{C}(40)$ & $119.3(3)$ \\
\hline $\mathrm{C}(26)-\mathrm{C}(27)-\mathrm{C}(22)$ & $119.7(3)$ & $\mathrm{C}(38)-\mathrm{C}(39)-\mathrm{H}(39)$ & 120.3 \\
\hline $\mathrm{O}(4)-\mathrm{C}(28)-\mathrm{H}(28 \mathrm{~A})$ & 109.5 & $\mathrm{C}(40)-\mathrm{C}(39)-\mathrm{H}(39)$ & 120.3 \\
\hline $\mathrm{O}(4)-\mathrm{C}(28)-\mathrm{H}(28 \mathrm{~B})$ & 109.5 & $\mathrm{C}(41)-\mathrm{C}(40)-\mathrm{C}(39)$ & $121.8(3)$ \\
\hline $\mathrm{H}(28 \mathrm{~A})-\mathrm{C}(28)-\mathrm{H}(28 \mathrm{~B})$ & 109.5 & $\mathrm{C}(41)-\mathrm{C}(40)-\mathrm{H}(40)$ & 119.1 \\
\hline $\mathrm{O}(4)-\mathrm{C}(28)-\mathrm{H}(28 \mathrm{C})$ & 109.5 & $\mathrm{C}(39)-\mathrm{C}(40)-\mathrm{H}(40)$ & 119.1 \\
\hline $\mathrm{H}(28 \mathrm{~A})-\mathrm{C}(28)-\mathrm{H}(28 \mathrm{C})$ & 109.5 & $\mathrm{C}(40)-\mathrm{C}(41)-\mathrm{C}(36)$ & $117.5(3)$ \\
\hline $\mathrm{H}(28 \mathrm{~B})-\mathrm{C}(28)-\mathrm{H}(28 \mathrm{C})$ & 109.5 & $\mathrm{C}(40)-\mathrm{C}(41)-\mathrm{Cr}(1)$ & $129.2(2)$ \\
\hline N(1)-C(29)-H(29A) & 109.5 & $\mathrm{C}(36)-\mathrm{C}(41)-\mathrm{Cr}(1)$ & $113.3(2)$ \\
\hline $\mathrm{N}(1)-\mathrm{C}(29)-\mathrm{H}(29 \mathrm{~B})$ & 109.5 & $\mathrm{Cl}(1)-\mathrm{C}(51)-\mathrm{Cl}(2)$ & 109.1(5) \\
\hline $\mathrm{H}(29 \mathrm{~A})-\mathrm{C}(29)-\mathrm{H}(29 \mathrm{~B})$ & 109.5 & $\mathrm{Cl}(1)-\mathrm{C}(51)-\mathrm{H}(51 \mathrm{~A})$ & 109.9 \\
\hline N(1)-C(29)-H(29C) & 109.5 & $\mathrm{Cl}(2)-\mathrm{C}(51)-\mathrm{H}(51 \mathrm{~A})$ & 109.9 \\
\hline $\mathrm{H}(29 \mathrm{~A})-\mathrm{C}(29)-\mathrm{H}(29 \mathrm{C})$ & 109.5 & $\mathrm{Cl}(1)-\mathrm{C}(51)-\mathrm{H}(51 \mathrm{~B})$ & 109.9 \\
\hline $\mathrm{H}(29 \mathrm{~B})-\mathrm{C}(29)-\mathrm{H}(29 \mathrm{C})$ & 109.5 & $\mathrm{Cl}(2)-\mathrm{C}(51)-\mathrm{H}(51 \mathrm{~B})$ & 109.9 \\
\hline $\mathrm{C}(31)-\mathrm{C}(30)-\mathrm{C}(35)$ & $117.6(3)$ & $\mathrm{H}(51 \mathrm{~A})-\mathrm{C}(51)-\mathrm{H}(51 \mathrm{~B})$ & 108.3 \\
\hline $\mathrm{C}(31)-\mathrm{C}(30)-\mathrm{Cr}(1)$ & $128.5(2)$ & Cl(2B)-C(51B)-Cl(1B) & $115(4)$ \\
\hline $\mathrm{C}(35)-\mathrm{C}(30)-\mathrm{Cr}(1)$ & $113.6(2)$ & $\mathrm{Cl}(2 \mathrm{~B})-\mathrm{C}(51 \mathrm{~B})-\mathrm{H}(51 \mathrm{C})$ & 108.6 \\
\hline $\mathrm{C}(32)-\mathrm{C}(31)-\mathrm{C}(30)$ & $122.6(3)$ & $\mathrm{Cl}(1 \mathrm{~B})-\mathrm{C}(51 \mathrm{~B})-\mathrm{H}(51 \mathrm{C})$ & 108.6 \\
\hline $\mathrm{C}(32)-\mathrm{C}(31)-\mathrm{H}(31)$ & 118.7 & $\mathrm{Cl}(2 \mathrm{~B})-\mathrm{C}(51 \mathrm{~B})-\mathrm{H}(51 \mathrm{D})$ & 108.6 \\
\hline $\mathrm{C}(30)-\mathrm{C}(31)-\mathrm{H}(31)$ & 118.7 & Cl(1B)-C(51B)-H(51D) & 108.6 \\
\hline $\mathrm{C}(31)-\mathrm{C}(32)-\mathrm{C}(33)$ & $119.0(3)$ & $\mathrm{H}(51 \mathrm{C})-\mathrm{C}(51 \mathrm{~B})-\mathrm{H}(51 \mathrm{D})$ & 107.6 \\
\hline
\end{tabular}


Table 16. Anisotropic displacement parameters $\left(\AA^{2} \times 10^{4}\right)$ for 4 (CCDC 208405). The anisotropic displacement factor exponent takes the form: $-2 \square^{2}\left[h^{2} a^{* 2} U{ }^{11}+\ldots+\right.$ $\left.2 \mathbf{h ~ k ~ a *} \mathbf{b}^{*} \mathbf{U}^{12}\right]$

\begin{tabular}{|c|c|c|c|c|c|c|}
\hline & $\mathrm{U}^{11}$ & $\mathrm{U}^{22}$ & $\mathrm{U}^{33}$ & $\mathrm{U}^{23}$ & $\mathrm{U}^{13}$ & $\mathrm{U}^{12}$ \\
\hline $\operatorname{Cr}(1)$ & $114(2)$ & $142(3)$ & 196(3) & $-37(2)$ & $-45(2)$ & $-11(2)$ \\
\hline $\operatorname{Br}(1)$ & 193(2) & $196(2)$ & $298(2)$ & $-52(1)$ & $-83(1)$ & $-61(1)$ \\
\hline $\mathrm{P}(1)$ & $143(4)$ & $131(4)$ & $159(4)$ & $-28(3)$ & $-22(3)$ & $-28(3)$ \\
\hline $\mathrm{P}(2)$ & $158(4)$ & 133(4) & 157(4) & $-28(3)$ & $-22(3)$ & $-29(3)$ \\
\hline $\mathrm{O}(1)$ & $139(10)$ & $106(10)$ & $224(11)$ & $-14(8)$ & $-65(8)$ & $-44(8)$ \\
\hline $\mathrm{O}(2)$ & $168(10)$ & $164(11)$ & $286(12)$ & $-53(9)$ & 20(9) & $-19(9)$ \\
\hline $\mathrm{O}(3)$ & 249(11) & $300(12)$ & $227(12)$ & $-23(10)$ & $-69(9)$ & $-148(10)$ \\
\hline $\mathrm{O}(4)$ & $169(11)$ & $231(12)$ & $322(13)$ & $-48(10)$ & 63(9) & $-57(9)$ \\
\hline $\mathrm{N}(1)$ & $122(12)$ & $169(13)$ & $188(13)$ & $-65(10)$ & $-8(10)$ & $-28(10)$ \\
\hline $\mathrm{C}(1)$ & $84(13)$ & $139(15)$ & $173(16)$ & $-26(12)$ & $-18(12)$ & $9(11)$ \\
\hline $\mathrm{C}(2)$ & $158(14)$ & $168(16)$ & $224(17)$ & $-46(13)$ & $-25(13)$ & $-60(12)$ \\
\hline $\mathrm{C}(3)$ & $156(15)$ & $214(17)$ & 209(17) & $-66(14)$ & $-54(13)$ & $-45(13)$ \\
\hline $\mathrm{C}(4)$ & $126(14)$ & $262(17)$ & $184(16)$ & $-44(14)$ & $-53(12)$ & $6(13)$ \\
\hline$C(5)$ & $112(14)$ & $134(15)$ & $232(17)$ & $-17(13)$ & $-9(12)$ & $-17(12)$ \\
\hline$C(6)$ & $57(13)$ & $157(15)$ & $170(16)$ & $-53(12)$ & $-17(11)$ & 7(11) \\
\hline $\mathrm{C}(7)$ & $186(15)$ & $144(16)$ & $227(17)$ & $35(13)$ & $-35(13)$ & $-67(13)$ \\
\hline $\mathrm{C}(8)$ & 208(15) & $172(16)$ & $174(16)$ & $-43(13)$ & $-63(13)$ & $-61(13)$ \\
\hline C(9) & 241(16) & $195(17)$ & 241(18) & $-31(14)$ & $-90(14)$ & $-77(13)$ \\
\hline$C(10)$ & $295(18)$ & 261(18) & 194(17) & $41(14)$ & $-54(14)$ & $-143(15)$ \\
\hline $\mathrm{C}(11)$ & 393(19) & $167(17)$ & 239(18) & $34(14)$ & $-151(15)$ & $-117(15)$ \\
\hline$C(12)$ & $256(17)$ & $161(16)$ & 278(19) & $-70(14)$ & $-99(14)$ & $-35(13)$ \\
\hline $\mathrm{C}(13)$ & $219(16)$ & 205(17) & $174(16)$ & $-6(13)$ & $-62(13)$ & $-95(13)$ \\
\hline$C(14)$ & $169(15)$ & 192(17) & $380(20)$ & $-88(15)$ & $-18(14)$ & 1(13) \\
\hline$C(15)$ & 208(15) & $159(16)$ & $162(16)$ & $-33(13)$ & $-20(13)$ & $-20(13)$ \\
\hline$C(16)$ & $540(20)$ & $310(20)$ & 205(19) & $-26(15)$ & $-49(17)$ & $-213(17)$ \\
\hline $\mathrm{C}(17)$ & $600(20)$ & $370(20)$ & $290(20)$ & $15(17)$ & $-28(18)$ & $-286(19)$ \\
\hline $\mathrm{C}(18)$ & $600(20)$ & $380(20)$ & $164(18)$ & 1(16) & $-55(17)$ & $-216(19)$ \\
\hline $\mathrm{C}(19)$ & $278(17)$ & $258(18)$ & 198(18) & $-54(14)$ & $-41(14)$ & $-72(14)$ \\
\hline$C(20)$ & $136(14)$ & $152(16)$ & 208(17) & $-30(13)$ & $-15(12)$ & 1(12) \\
\hline $\mathrm{C}(21)$ & $400(20)$ & $530(20)$ & $330(20)$ & $18(18)$ & $-149(17)$ & $-326(19)$ \\
\hline$C(22)$ & $174(15)$ & $162(16)$ & $142(16)$ & $-42(12)$ & $-19(12)$ & $-26(12)$ \\
\hline $\mathrm{C}(23)$ & $163(14)$ & $183(16)$ & 189(16) & $-34(13)$ & $-69(12)$ & $-50(12)$ \\
\hline$C(24)$ & $313(18)$ & $153(16)$ & 251(18) & $-32(14)$ & $-75(14)$ & $-75(14)$ \\
\hline$C(25)$ & $246(17)$ & $165(17)$ & $340(20)$ & $-85(15)$ & $-123(15)$ & $48(14)$ \\
\hline$C(26)$ & $150(15)$ & 276(19) & 271(18) & $-107(15)$ & $-40(13)$ & $-3(14)$ \\
\hline$C(27)$ & 199(15) & 202(17) & $173(16)$ & $-69(13)$ & $-21(13)$ & $-36(13)$ \\
\hline$C(28)$ & $184(16)$ & $430(20)$ & 286(19) & $-133(16)$ & $62(14)$ & $-131(15)$ \\
\hline$C(29)$ & $139(15)$ & $235(17)$ & 288(18) & $-89(14)$ & $-17(13)$ & $-66(13)$ \\
\hline$C(30)$ & $171(15)$ & 208(17) & $237(17)$ & $-57(14)$ & $-70(13)$ & $-25(13)$ \\
\hline $\mathrm{C}(31)$ & 231(17) & $300(19)$ & 270(19) & $-58(15)$ & $-86(14)$ & $48(14)$ \\
\hline$C(32)$ & $317(19)$ & $350(20)$ & 261(19) & $-53(16)$ & $-119(15)$ & $21(16)$ \\
\hline$C(33)$ & $280(18)$ & $233(18)$ & $330(20)$ & $26(15)$ & $-182(15)$ & $-18(14)$ \\
\hline$C(34)$ & $172(15)$ & $157(16)$ & $380(20)$ & $-65(14)$ & $-82(14)$ & $-3(13)$ \\
\hline$C(35)$ & $135(14)$ & $133(15)$ & 283(18) & $-36(13)$ & $-68(13)$ & $-34(12)$ \\
\hline$C(36)$ & $129(14)$ & $178(16)$ & $287(18)$ & $-59(14)$ & $-40(13)$ & $-69(12)$ \\
\hline$C(37)$ & $116(14)$ & $180(17)$ & $360(20)$ & $-91(14)$ & $3(14)$ & $-27(12)$ \\
\hline$C(38)$ & $133(15)$ & 199(17) & $420(20)$ & $-146(15)$ & $97(14)$ & $-50(13)$ \\
\hline
\end{tabular}




\begin{tabular}{lllllll}
$\mathrm{C}(39)$ & $236(16)$ & $239(17)$ & $201(17)$ & $-51(14)$ & $13(13)$ & $-132(14)$ \\
$\mathrm{C}(40)$ & $148(15)$ & $171(16)$ & $293(19)$ & $-43(14)$ & $-46(13)$ & $-50(12)$ \\
$\mathrm{C}(41)$ & $125(14)$ & $130(15)$ & $221(17)$ & $-26(12)$ & $-38(12)$ & $-63(12)$ \\
& & & & & & \\
$\mathrm{C}(51)$ & $370(50)$ & $260(30)$ & $310(40)$ & $-70(30)$ & $-110(30)$ & $-10(30)$ \\
$\mathrm{Cl}(1)$ & $380(6)$ & $263(6)$ & $316(6)$ & $-87(5)$ & $-134(5)$ & $42(5)$ \\
$\mathrm{Cl}(2)$ & $402(6)$ & $232(6)$ & $424(7)$ & $-105(5)$ & $-71(5)$ & $-16(5)$ \\
& & & & & \\
$\mathrm{C}(51 \mathrm{~B})$ & $700(400)$ & $1500(500)$ & $900(400)$ & $-100(300)$ & $-100(300)$ & $-700(400)$ \\
$\mathrm{Cl}(1 \mathrm{~B})$ & $1570(80)$ & $1140(70)$ & $1260(80)$ & $-90(60)$ & $-680(70)$ & $-540(60)$ \\
$\mathrm{Cl}(2 \mathrm{~B})$ & $1480(90)$ & $1250(80)$ & $1110(70)$ & $-510(60)$ & $320(70)$ & $-200(70)$ \\
& & & & & \\
\hline
\end{tabular}




\section{References}

(1) Pangborn, A. B.; Giardello, M. A.; Grubbs, R. H.; Rosen, R. K.; Timmers, F. J. Organometallics 1996, 15, 1518.

(2) Cooley, N. A.; Green, S. M.; Wass, D. F.; Heslop, K.; Orpen, A. G.; Pringle, P. G. Organometallics 2001, 20, 4769.

(3) Herwig, W.; Zeiss, H. J. Am. Chem. Soc. 1959, 81, 4798.

(4) IsoCalc(TM) version 2.2, ChemSW, http://www.chemsw.com. 